\title{
Radical Chain Reduction via Carbon Dioxide Radical Anion $\left(\mathrm{CO}_{2}{ }^{--}\right)$
}

\author{
Cecilia M. Hendy, ${ }^{\dagger}$ Gavin C. Smith ${ }^{\dagger}$ Zihao Xu, Tianquan Lian, Nathan T. Jui* \\ njui@emory.edu
}

Department of Chemistry and Winship Cancer Institute, Emory University, Atlanta, GA 30322

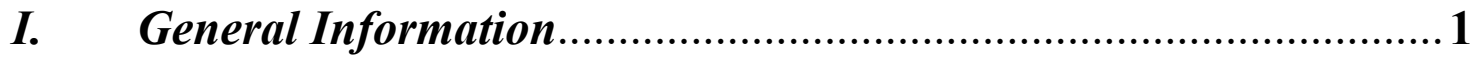

a. General Reagent Information .............................................

b. General Analytical Information.......................................... 1

c. Abbreviations............................................................. 1

d. General Photoredox Setup..................................................2

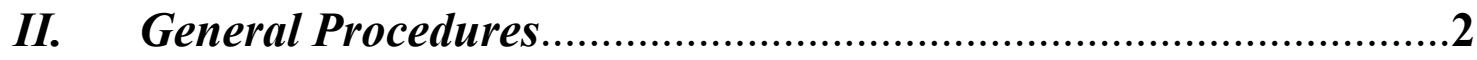

a. General Procedure A ........................................................ 2

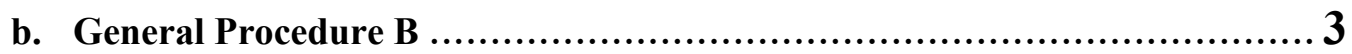

III. Optimization Details.........................................................4

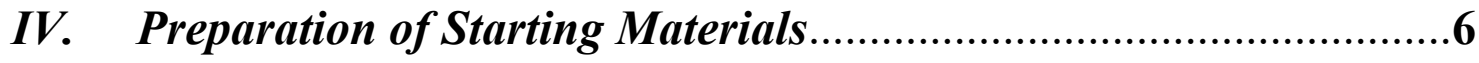

V. Preparation of Products from Substrate Table .....................12

VI. Carboxylation/Reduction of Olefins .......................................22

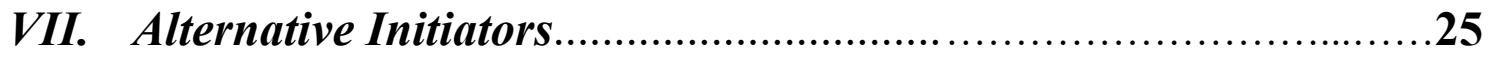

VIII. Fluorescence Quenching Experiments..............................26

IX. Transient Absorption Spectroscopy ....................................27

X. Quantum Yield Experiments............................................31

XI. Chain Length Approximation ................................... 33

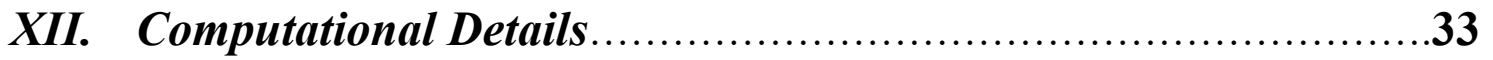

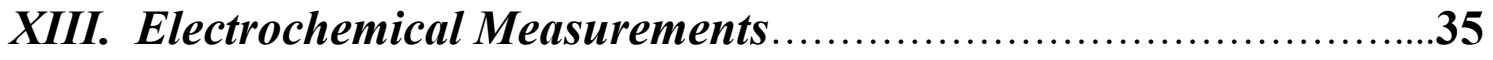

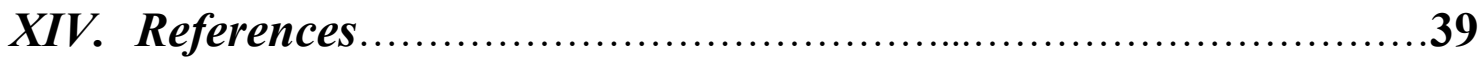

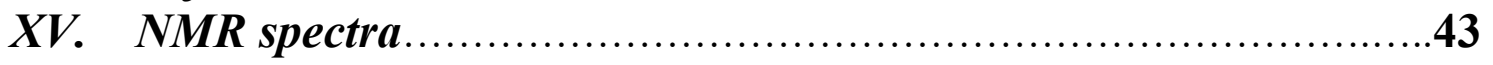




\section{General Information}

\section{I-A. General Reagent Information}

Reagents were purchased from Sigma-Aldrich, Alfa Aesar, Acros Organics, Combi-Blocks, Oakwood Chemicals, Astatech, and TCI America and used as received, unless stated otherwise. All reactions were set up on the bench top and conducted under nitrogen atmosphere while subject to irradiation from blue LEDs (LEDwholesalers PAR38 Indoor Outdoor 16-Watt LED Flood Light Bulb, Blue; or Hydrofarm ${ }^{\circledR}$ PPB1002 PowerPAR LED Bulb-Blue 15W/E27 (available from Amazon); or a Kessil® PR160L- $390 \mathrm{~nm}$. Flash chromatography was carried out using Siliaflash ${ }^{\circledR}$ P60 silica gel obtained from Silicycle. Thin-layer chromatography (TLC) was performed on $250 \mu \mathrm{m}$ SiliCycle silica gel F-254 plates. Visualization of the developed chromatogram was performed by fluorescence quenching or staining using $\mathrm{KMnO}_{4}$, panisaldehyde, or ninhydrin stains. DMSO was purchased from Fisher Scientific and was distilled over $\mathrm{CaH} 2$ and degassed by sonication under vacuum and stored under nitrogen. Photoredox catalyst $4 \mathrm{CzIPN}$ was prepared according to literature procedures. ${ }^{1}$

\section{I-B. General Analytical Information.}

Unless otherwise noted, all yields refer to chromatographically and spectroscopically $\left({ }^{1} \mathrm{H}\right.$ NMR) homogenous materials. New compounds were characterized by NMR and HRMS. ${ }^{1} \mathrm{H}$ and ${ }^{13} \mathrm{C}$ NMR spectra were obtained from the Emory University NMR facility and recorded on a Bruker Avance III HD 600 equipped with cryo-probe (600 MHz), Bruker 400 (400 MHz), INOVA 600 (600 MHz), INOVA 500 (500 MHz), INOVA 400 (400 MHz), or VNMR 400 (400 MHz), and are internally referenced to residual protio solvent signals. Data for ${ }^{1} \mathrm{H}$ NMR are reported as follows: chemical shift ( $\mathrm{ppm})$, multiplicity $(\mathrm{s}=$ singlet, $\mathrm{d}=$ doublet, $\mathrm{t}=$ triplet, $\mathrm{q}=$ quartet, $\mathrm{m}=$ multiplet, $\mathrm{dd}=$ doublet of doublets, $\mathrm{dt}=$ doublet of triplets, $\mathrm{ddd}=$ doublet of doublet of doublets, $\mathrm{dtd}=$ doublet of triplet of doublets, $\mathrm{b}=$ broad, etc.), coupling constant $(\mathrm{Hz})$, integration, and assignment, when applicable. Data for decoupled ${ }^{13} \mathrm{C}$ NMR are reported in terms of chemical shift and multiplicity when applicable. High Resolution mass spectra were obtained from the Emory University Mass Spectral facility.

\section{I-C. Abbreviations.}

DMSO = dimethyl sulfoxide

$\mathrm{DMF}=$ dimethylformamide

$\mathrm{THF}=$ tetrahydrofuran

$\mathrm{DCM}=$ dichloromethane

chromatography

TLC $=$ thin layer chromatograph

$\mathrm{TEA}=$ triethylamine

EtOAc $=$ ethyl acetate

$\mathrm{MeCN}=$ acetonitrile

LCMS = liquid chromatography mass spectrometry

GCMS = gas chromatography mass spectrometry 
$\mathrm{MeOH}=$ methanol

AIBN= Azobisisobutyronitrile

\section{I-D. General Photoredox Reaction Setup}

To run multiple reactions, an appropriately sized 3D printed carousel was used, which exposed the reactions to the blue light evenly (photo 1). A $15 \mathrm{~W}$ LED array lamp was used as a blue light source (photo 2,3). These lamps were routinely used for up to 12 reactions at a time (photo 2,3). The blue LEDs were positioned approximately 6 inches above the reaction vials to get good light coverage without overheating the reactions (photo 2,3). Reactions run at elevated temperatures were irradiated in a shallow oil bath (photo 4,5).

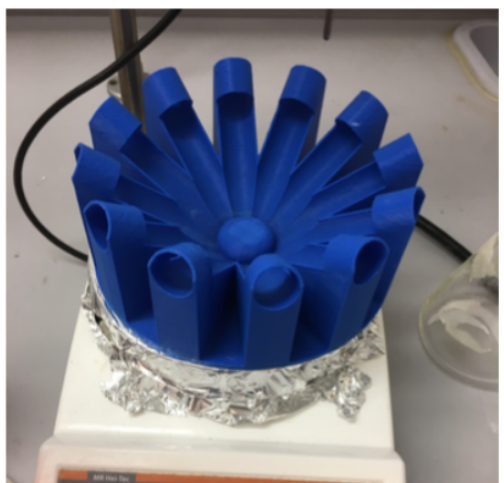

Photo 1

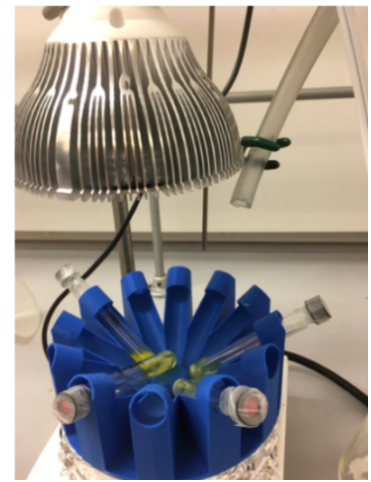

Photo 2

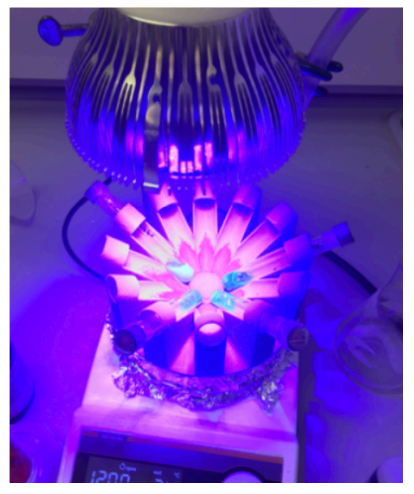

Photo 3

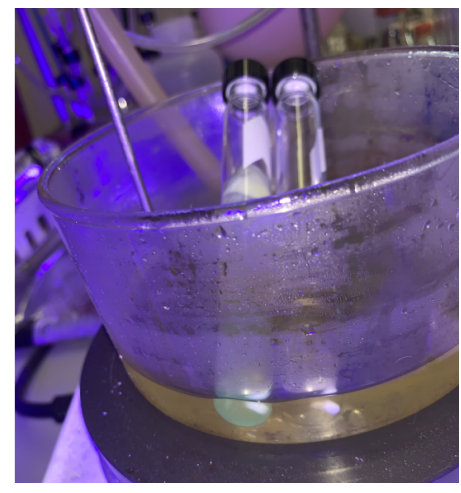

Photo 4

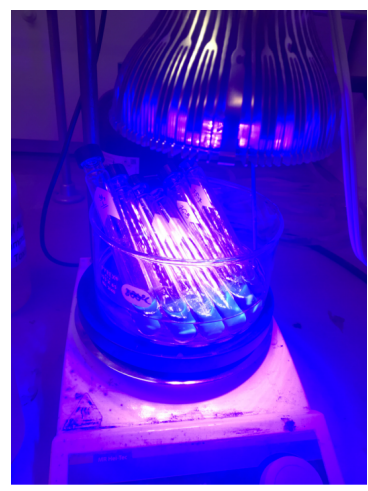

Photo 5

\section{General Procedures}

\section{General Procedure A}

A $20 \mathrm{~mL}$ screw-top test tube was charged with 4CzIPN (1 mol\%), sodium formate (5.0 equiv), mesna (20 mol\%), arene (if solid, 1.0 equiv) and alkene (if solid, 5.0 equiv). The tube was equipped with a stir bar and was sealed with a PTFE/silicon septum. The atmosphere was exchanged by applying vacuum and backfilling with nitrogen (this process was conducted a total of three times). Under nitrogen atmosphere, separately the indicated degassed solvent $(0.1 \mathrm{M})$ 
was added via syringe followed by the arene (if liquid, 1.0 equiv) and the alkene (if liquid, 5.0 equiv). The resulting mixture was stirred at $1400 \mathrm{RPM}$ for $16 \mathrm{~h}$ under irradiation by blue LEDs. Water was added and then the reaction mixture was extracted with ethyl acetate $(3 \mathrm{x})$. The organic layer was passed through a small silica plug with $100 \%$ EtOAc and concentrated. The residue was purified by silica chromatography using the indicated solvent mixture as the eluent to afford the title compound.

\section{General Procedure B}

A $20 \mathrm{~mL}$ screw-top test tube was charged with 4CzIPN ( $1 \mathrm{~mol} \%$ ), sodium formate (5.0 equiv), mesna (20 mol\%), substrate (if solid, 1.0 equiv). The tube was equipped with a stir bar and was sealed with a PTFE/silicon septum. The atmosphere was exchanged by applying vacuum and backfilling with nitrogen (this process was conducted a total of three times). Under nitrogen atmosphere, separately the indicated degassed solvent $(0.1 \mathrm{M})$ was added via syringe followed by the substrate (if liquid, 1.0 equiv). The resulting mixture was stirred at 1400 RPM for $16 \mathrm{~h}$ under irradiation by blue LEDs. Water was added and then the reaction mixture was extracted with ethyl acetate $(3 \mathrm{x})$. The organic layer was passed through a small silica plug with $100 \%$ EtOAc and concentrated. The residue was purified by silica chromatography using the indicated solvent mixture as the eluent to afford the title compound. 


\section{Optimization Details}

\section{Optimization Procedure}

An $8 \mathrm{~mL}$ screw-top test tube was charged with 4CzIPN ( $1 \mathrm{~mol} \%, 0.001 \mathrm{~mol})$, sodium formate (5.0 equiv, $0.5 \mathrm{mmol})$ and mesna $(20 \mathrm{~mol} \%, 0.02 \mathrm{~mol})$. The tube was equipped with a stir bar and sealed with a PTFE/silicon septum. Under nitrogen atmosphere, separately degassed DMSO $(1 \mathrm{~mL})$ was added via syringe, followed by 2 -chloromethylbenzoate ( 1 equiv, $0.1 \mathrm{mmol}$ ). The resulting mixture was stirred at $1400 \mathrm{RPM}$ for $16 \mathrm{~h}$ under irradiation by blue LEDs. The reaction was then extracted with ethyl acetate $(3 \mathrm{x})$. The organic layer was passed through a small silica plug with 100\% EtOAc and concentrated. Deutro-chloroform with an internal standard of dibromomethane $(7 \mu \mathrm{L}, 0.1 \mathrm{mmol})$ was added. The sample was analyzed by ${ }^{1} \mathrm{H}$ NMR $(\mathrm{d}=5 \mathrm{~s})$, and the integral values were used to calculate the data given in Table S1-S3.

\section{HAT catalysts:}

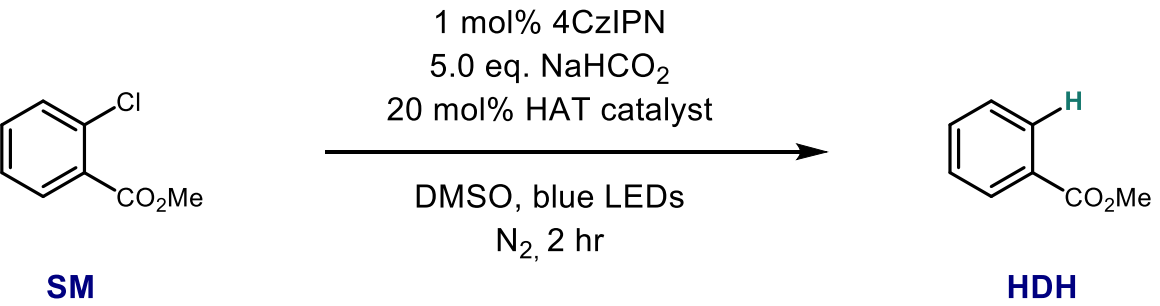

\begin{tabular}{lccc}
\hline Entry & HAT catalyst & SM & HDH \\
\hline 1 & none & 97 & 0 \\
2 & mesna & 0 & 100 \\
3 & cyclohexane thiol & 0 & 91 \\
4 & dodecane thiol & 0 & 93 \\
5 & thiophenol & 0 & 92 \\
6 & thiobenzoic acid & 0 & 92 \\
7 & DABCo & 39 & 62 \\
\hline & yields determined by ${ }^{1} \mathrm{H}$ NMR with dibromomethane as internal standard
\end{tabular}

Table S1 
Varying Photocatalysts:

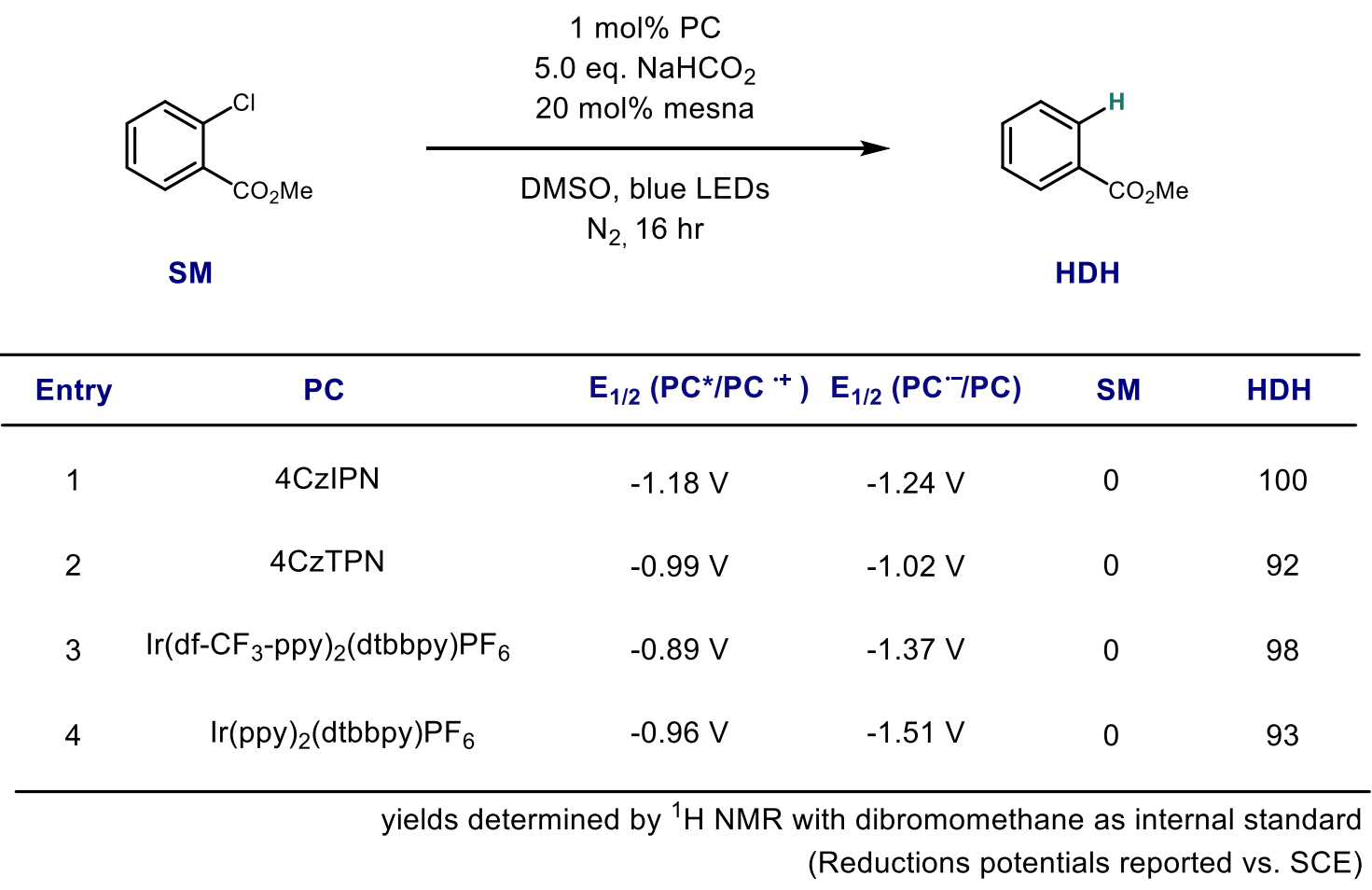

Table S2.

\section{Controls:}

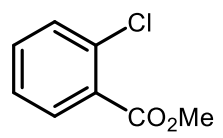

SM initiator

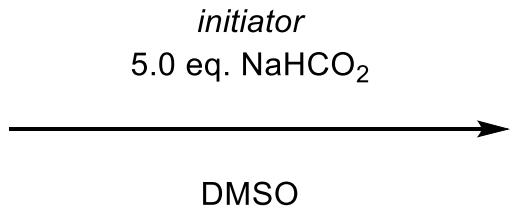

$\mathrm{N}_{2}, 16 \mathrm{hr}$

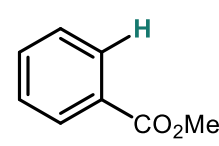

$\mathrm{HDH}$

\begin{tabular}{cccc}
\hline Entry & deviations & SM & HDH \\
\hline 1 & no 4CzIPN & 100 & 0 \\
2 & no formate & 99 & 0 \\
3 & no light & 102 & 0 \\
4 & in presence of air & 3 & 94 \\
\hline
\end{tabular}

yields determined by ${ }^{1} \mathrm{H}$ NMR with dibromomethane as internal standard Table S3 


\section{Preparation of Starting Materials}<smiles>C=CN(C=O)C(=O)c1ccccc1</smiles>

Tert-butyl formyl(vinyl)carbamate (S1): The title compound was prepared according to literature procedure. The physical properties and spectral data were consistent with reported values. $^{2}$

${ }^{1}$ H NMR (600 MHz, Chloroform-d) $\delta 9.26(\mathrm{~s}, 1 \mathrm{H}), 6.59(\mathrm{dd}, \mathrm{J}=16.3,9.7 \mathrm{~Hz}, 1 \mathrm{H}), 5.67$ (d, J = $16.2 \mathrm{~Hz}, 1 \mathrm{H}), 5.06(\mathrm{~d}, \mathrm{~J}=9.6 \mathrm{~Hz}, 1 \mathrm{H}),(\mathrm{s}, 12 \mathrm{H})$.<smiles>C=CNC(=O)OCc1ccccc1</smiles>

Tert-butyl vinylcarbamate (S2): The title compound was prepared according to literature procedure. The physical properties and spectral data were consistent with reported values. ${ }^{2}$ ${ }^{1}$ H NMR (600 MHz, DMSO-d 6 ) $\delta(9.20, \mathrm{~s}, 1 \mathrm{H}), 6.51$ (ddd, J = 15.9, 10.4, 8.9 Hz, 1H), 4.45 (d, $\mathrm{J}=15.9 \mathrm{~Hz}, 1 \mathrm{H}), 4.09(\mathrm{~d}, \mathrm{~J}=8.9 \mathrm{~Hz}, 1 \mathrm{H}), 1.41(\mathrm{~s}, 9 \mathrm{H})$.<smiles>CCOP(=O)(OCC)c1cccc(Br)c1</smiles>

Diethyl (3-bromophenyl)phosphonate (S3): The title compound was prepared according to literature procedure. The physical properties and spectral data were consistent with reported values. $^{3}$

${ }^{1}$ H NMR (500 MHz, Chloroform-d) $\delta 7.94$ (ddd, $\left.J=13.6,1.9,1.4 \mathrm{~Hz}, 1 \mathrm{H}\right), 7.73$ (ddt, $J=12.9$, 7.6, 1.2 Hz, 1H), 7.67 (ddt, $J=8.1,2.1,1.0 \mathrm{~Hz}, 1 \mathrm{H}), 7.34(\mathrm{td}, J=7.8,4.7 \mathrm{~Hz}, 1 \mathrm{H}), 4.11(\mathrm{~m}, 4 \mathrm{H})$, $1.32(\mathrm{t}, \mathrm{J}=7.1 \mathrm{~Hz}, 6 \mathrm{H})$.<smiles>CN(C)c1cccc(C#N)c1</smiles>

3-(dimethylamino)benzonitrile (S4): A round bottom flask equipped with a stirbar was added 3-aminobenzonitrile (10 mmol, $1.2 \mathrm{~g}, 1.0$ equiv), $\mathrm{Na}_{2} \mathrm{CO}_{3}(20 \mathrm{mmol}, 2.1 \mathrm{~g}, 2.0$ equiv) and DMF $(10 \mathrm{~mL})$. Methyl iodide ( $25 \mathrm{mmol}, 1.6 \mathrm{~mL}, 2.5$ equiv) was added dropwise to the solution a few minutes later. The reaction was heated to $80^{\circ} \mathrm{C}$ and stirred overnight. The reaction was allowed to cool to room temp. and it was diluted in EtOAc. The solution was washed with a solution of 1 $\mathrm{M} \mathrm{LiCl}$, extracted with EtOAc $(3 \mathrm{x})$, dried over $\mathrm{Na}_{2} \mathrm{SO}_{4}$ and concentrated in vacuo. The crude reaction mixture was pushed through a silica plug (10\% EtOAc/hexanes) to afford the title compound as an orange oil (1.0 g, 69\% yield). The spectral properties were consistent with the reported values. ${ }^{4}$

${ }^{1}$ H NMR (500 MHz, Chloroform-d) $\delta 7.32-7.26(\mathrm{~m}, 1 \mathrm{H}), 6.95$ (dt, $\left.J=7.5,1.1 \mathrm{~Hz}, 2 \mathrm{H}\right), 6.91$ $-6.82(\mathrm{~m}, 1 \mathrm{H}), 2.98(\mathrm{~s}, 6 \mathrm{H})$. 


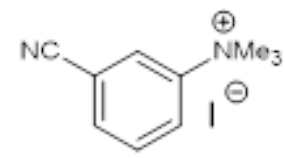

3-cyano-N,N,N-trimethylbenzenaminium iodide (S5): The procedure used was adapted from Wang. ${ }^{5}$ A round bottom flask equipped with a stir bar was added methyl 3-

(dimethylamino)benzonitrile $\mathbf{S 4}$ (6.0 mmol, $0.88 \mathrm{~g}, 1.0$ equiv) and DMF (4 mL). Methyl iodide ( $12 \mathrm{mmol}, 0.74 \mathrm{~mL}, 2.0$ equiv) was added dropwise. The reaction was heated to $50^{\circ} \mathrm{C}$ and stirred overnight. The reaction was allowed to cool to room temp. and it was diluted with $\mathrm{Et}_{2} \mathrm{O}$. The solid precipitate was collected by vacuum filtration to afford the title compound as a white solid (1.26 g, 73\% yield).

${ }^{1}$ H NMR (500 MHz, DMSO- $\left.\boldsymbol{d}_{\mathbf{6}}\right) \delta 8.58(\mathrm{dd}, J=2.4,1.3 \mathrm{~Hz}, 1 \mathrm{H}), 8.34(\mathrm{ddd}, J=8.7,2.8,0.9 \mathrm{~Hz}$, $1 \mathrm{H}), 8.15-8.06(\mathrm{~m}, 1 \mathrm{H}), 7.90-7.85(\mathrm{~m}, 1 \mathrm{H}), 3.64(\mathrm{~s}, 9 \mathrm{H})$.

${ }^{13}$ C NMR (151 MHz, DMSO-d 6 ) $\delta$ 147.43, 133.94, 131.33, 125.86, 125.13, 117.47, 112.81, 56.47 .

HRMS (APCI) $m / z$ : [M+] calcd. for $\mathrm{C}_{10} \mathrm{H}_{13} \mathrm{~N}_{2}, 161.0709$; found, 161.0711. [M-] calcd. for I, 126.9050; found, 126.9057 .<smiles>CCOP(=O)(OCC)c1cccc(N(C)C)c1</smiles>

diethyl (3-(dimethylamino)phenyl)phosphonate (S6): The title compound was prepared according to literature procedure. The physical properties and spectral data were consistent with reported values. ${ }^{6}$

${ }^{1}$ H NMR (400 MHz, Chloroform-d) $\delta 7.36-7.26(\mathrm{~m}, 1 \mathrm{H}), 7.20-7.05$ (m, 2H), 6.87 (d, J = 8.4 $\mathrm{Hz}, 1 \mathrm{H}), 4.20-3.99(\mathrm{~m}, 4 \mathrm{H}), 2.98(\mathrm{~s}, 6 \mathrm{H}), 1.32(\mathrm{t}, \mathrm{J}=7.1 \mathrm{~Hz}, 6 \mathrm{H})$.<smiles>CCOP(=O)(OCC)c1cccc([N+](=O)[O-])c1</smiles>

3-(diethoxyphosphoryl)-N,N,N-trimethylbenzenaminium iodide (S7): The procedure used was adapted from Wang. ${ }^{5}$ A round bottom flask equipped with a stir bar was added diethyl (3(dimethylamino)phenyl)phosphonate $\mathbf{S 6}(2.0 \mathrm{mmol}, 0.51 \mathrm{~g}, 1.0$ equiv) and DMF (1.5 mL). Methyl iodide ( $4 \mathrm{mmol}, 0.25 \mathrm{~mL}, 2.0$ equiv) was added dropwise. The reaction was heated to 50 ${ }^{\circ} \mathrm{C}$ and stirred overnight. The reaction was allowed to cool to room temp. and it was diluted with $\mathrm{Et}_{2} \mathrm{O}$. The solid precipitate was collected by vacuum filtration to give the title compound as a white solid ( $0.65 \mathrm{~g}, 81 \%$ yield).

${ }^{1}$ H NMR (400 MHz, Methanol-d4) $\delta 8.26(\mathrm{dd}, \mathrm{J}=14.8,3.3 \mathrm{~Hz}, 2 \mathrm{H}), 8.02$ (ddt, J = 12.7, 7.5, $1.0 \mathrm{~Hz}, 1 \mathrm{H}), 7.94-7.83(\mathrm{~m}, 1 \mathrm{H}), 4.31-4.12(\mathrm{~m}, 4 \mathrm{H}), 3.77(\mathrm{~s}, 9 \mathrm{H}), 1.38(\mathrm{t}, \mathrm{J}=7.1 \mathrm{~Hz}, 6 \mathrm{H})$.

${ }^{13}$ C NMR (151 MHz, Methanol-d4) $\delta 148.80(\mathrm{~d}, \mathrm{~J}=18.9 \mathrm{~Hz}), 134.72(\mathrm{~d}, \mathrm{~J}=8.9 \mathrm{~Hz}), 132.56(\mathrm{~d}$, $\mathrm{J}=15.7 \mathrm{~Hz}), 132.52(\mathrm{~d}, \mathrm{~J}=192.1 \mathrm{~Hz}), 124.15(\mathrm{~d}, \mathrm{~J}=12.7 \mathrm{~Hz}), 64.56(\mathrm{~d}, \mathrm{~J}=6.0 \mathrm{~Hz}), 57.84$, $16.64(\mathrm{~d}, \mathrm{~J}=6.1 \mathrm{~Hz})$. 
${ }^{31}$ P NMR (162 MHz, Methanol-d4) $\delta 15.49$.

HRMS (APCI) $\mathrm{m} / z$ : [M+] calcd. for $\mathrm{C}_{13} \mathrm{H}_{23} \mathrm{NO}_{3} \mathrm{P}, 272.1410$; found, 272.1410. [M-] calcd. for I, 126.9050; found, 126.9056 .

$\mathrm{MeO}_{2} \mathrm{C} \mathrm{NMe}_{2}$

methyl 3-(dimethylamino)benzoate (S8): To a round bottom flask equipped with a stirbar was added 3-(dimethylamino)benzoic acid ( $20 \mathrm{mmol}, 3.3 \mathrm{~g}, 1.0$ equiv) and $\mathrm{MeOH}(20 \mathrm{~mL})$. A few drops of concentrated $\mathrm{HCl}$ was added and the reaction was refluxed overnight and then concentrated in vacuo. The crude product was diluted in EtOAc and washed with sat. $\mathrm{NaHCO}_{3}$ $(3 \mathrm{x})$, dried over $\mathrm{Na}_{2} \mathrm{SO}_{4}$ and concentrated in vacuo. The crude reaction mixture was pushed through a silica plug (5\% EtOAc) to give the title compound as a clear oil (1.7 g, 47\% yield). .) The physical and spectral properties were consistent with the reported values. ${ }^{7}$

${ }^{1}$ H NMR (500 MHz, Chloroform-d) $\delta 7.42-7.36(\mathrm{~m}, 2 \mathrm{H}), 7.29$ (t, $\left.J=7.8 \mathrm{~Hz}, 1 \mathrm{H}\right), 6.91$ (d, $J=$ $7.8 \mathrm{~Hz}, 1 \mathrm{H}), 3.90$ (s, 3H), 3.00 (s, 6H).<smiles>CC(=O)c1cccc([N+](=O)[O-])c1</smiles>

3-(methoxycarbonyl)-N,N,N-trimethylbenzenaminium iodide (S9): The procedure used was adapted from Wang. ${ }^{5}$ A round bottom flask equipped with a stir bar was added methyl 3(dimethylamino)benzoate $\mathbf{S 8}$ (9.0 mmol, $1.6 \mathrm{~g}, 1.0$ equiv) and DMF (7 mL). Methyl iodide (15 mmol, $0.93 \mathrm{~mL}, 1.5$ equiv) was added dropwise. The reaction was heated to $50{ }^{\circ} \mathrm{C}$ and stirred overnight. The reaction was allowed to cool to room temp. and it was diluted with $\mathrm{Et}_{2} \mathrm{O}$. The solid precipitate was collected by vacuum filtration to give the title compound as a white solid ( $2.4 \mathrm{~g}, 84 \%$ yield).

${ }^{1}$ H NMR (400 MHz, DMSO- $\left.\boldsymbol{d}_{\mathbf{6}}\right) \delta 8.43(\mathrm{dd}, J=2.8,1.4 \mathrm{~Hz}, 1 \mathrm{H}), 8.30$ (ddd, $J=8.6,2.9,0.9 \mathrm{~Hz}$, $1 \mathrm{H}), 8.14$ (ddd, $J=7.8,1.4,0.9 \mathrm{~Hz}, 1 \mathrm{H}), 7.82(\mathrm{t}, 1 \mathrm{H}), 3.92(\mathrm{~s}, 3 \mathrm{H}), 3.66(\mathrm{~s}, 9 \mathrm{H})$.

${ }^{13}$ C NMR (101 MHz, DMSO-d 6 ) $\delta$ 165.03, 147.48, 131.29, 130.83, 130.60, 125.55, 121.14, $56.40,52.81$.

HRMS (APCI) $m / z$ : [M+] calcd. for $\mathrm{C}_{11} \mathrm{H}_{16} \mathrm{NO}_{2}, 194.0812$; found, 194.0813. [M-] calcd. for I, 126.9050; found, 126.9056 .<smiles>[AlH2]N(c1ccccc1)c1ccccc1</smiles>

4-methyl-N,N-diphenylbenzenesulfonamide (S10): The title compound was prepared according to literature procedure. ${ }^{8}$ The physical and spectral properties were consistent with the reported values. ${ }^{9}$ 
${ }^{1}$ H NMR (600 MHz, Chloroform-d) $\delta 7.58(\mathrm{~d}, J=6.7 \mathrm{~Hz}, 2 \mathrm{H}), 7.35-7.22(\mathrm{~m}, 12 \mathrm{H}), 2.43(\mathrm{~s}$, $3 \mathrm{H}){ }^{1}$<smiles>[PbH3]c1cccnc1</smiles>

4-methyl- $N$-(pyridine-3-yl)benzenesulfonamide (S11 The title compound was prepared according to literature procedure. ${ }^{8}$ The physical and spectral properties were consistent with the reported values. ${ }^{10}$

${ }^{1}$ H NMR (400 MHz, DMSO-d 6 ): $\delta 10.49$ (bs, $\left.1 \mathrm{H}\right), 8.26$ (d, J = 2.6 Hz, 1H), 8.23 (dd, J = 4.7, J $=1.5 \mathrm{~Hz}, 1 \mathrm{H}), 7.65(\mathrm{~d}, \mathrm{~J}=8.3 \mathrm{~Hz}, 2 \mathrm{H}), 7.48(\mathrm{ddd}, \mathrm{J}=8.3,2.7,1.5 \mathrm{~Hz}, 1 \mathrm{H}), 7.35(\mathrm{~d}, \mathrm{~J}=8.1 \mathrm{~Hz}$, $1 \mathrm{H}), 7.27(\mathrm{dd}, \mathrm{J}=8.3,4.7 \mathrm{~Hz}, 1 \mathrm{H}), 2.33(\mathrm{~s}, 3 \mathrm{H})$.<smiles>[123I-]n1cnc2ccccc21</smiles>

1-tosyl-1H-benzo $[\boldsymbol{d}]$ imidazole (S12): The title compound was prepared according to literature procedure. ${ }^{8}$ The physical and spectral properties were consistent with the reported values. ${ }^{11}$

${ }^{1}$ H NMR (600 MHz, Chloroform-d) $\delta 8.39(\mathrm{~s}, 1 \mathrm{H}), 7.86(\mathrm{~m}, 3 \mathrm{H}), 7.76(\mathrm{~m}, 1 \mathrm{H}), 7.37(\mathrm{~m}, 2 \mathrm{H})$, $7.30(\mathrm{~m}, 2 \mathrm{H}), 2.38(\mathrm{~s}, 3 \mathrm{H})$.<smiles>O=CCCc1ccc(Cl)cc1</smiles>

3-(4-chlorophenyl)propanal (S13): To a round bottom flask was added 3-(4chlorophenyl)propan-1-ol (10 mmol, $1.5 \mathrm{~mL}, 1$ equiv), $\left[\mathrm{Cu}\left(\mathrm{CH}_{3} \mathrm{CN}\right)_{4}\right] \mathrm{PF}_{6}(0.5 \mathrm{mmol}, 0.19 \mathrm{~g}$, 0.05 equiv.), TEMPO ( $0.5 \mathrm{mmol}, 0.078 \mathrm{~g}, 0.05$ equiv.), 2,2-bipyridine ( $0.5 \mathrm{mmol}, 0.078 \mathrm{~g}, 0.05$ equiv), NMI ( $1 \mathrm{mmol}, 80 \mu \mathrm{L}, 0.1$ equiv) and $\mathrm{CH}_{3} \mathrm{CN}(50 \mathrm{~mL})$. The reaction was then sparged with air overnight then pushed through a silica plug using EtOAc and concentrated in vacuo. The crude product was then purified by silica chromatography $(0-30 \%$ EtOAc/Hexanes as the eluent) to give the title compound as a light yellow oil $(0.519 \mathrm{~g}, 31 \%$ yield.) The physical and spectral properties were consistent with the reported values. ${ }^{12}$

${ }^{1}$ H NMR (400 MHz, Chloroform-d) $\delta 9.80(\mathrm{t}, \mathrm{J}=1.3 \mathrm{~Hz}, 1 \mathrm{H})$, 7.27-7.21 (m, 2H), 7.15-7.08 (m, 2H), 2.96-2.87 (m, 2H), 2.80-2.73 (m, 2H).<smiles>O=C(c1cccc(C(F)(F)F)c1)N1CCOCC1</smiles>

morpholino(3-(trifluoromethyl)phenyl)methanone (S14): A round bottom flask equipped with a stir bar was added morpholine (11 mmol, $0.95 \mathrm{~mL}, 1.1$ equiv), triethylamine $(12.5 \mathrm{mmol}, 1.7 \mathrm{~g}$, 1.25 equiv) and $\mathrm{CH}_{2} \mathrm{Cl}_{2}(20 \mathrm{~mL})$ and stirred for 10 minutes. 3-(trifluoromethyl)benzoyl chloride 
(10 mmol, $1.5 \mathrm{~mL}, 1.0$ equiv) was added dropwise and the solution was allowed to stir for 1 hour. The reaction mixture was quenched with $1 \mathrm{M} \mathrm{HCl}$, extracted with $\mathrm{CH}_{2} \mathrm{Cl}_{2}(3 \mathrm{x})$, and concentrated in vacuo. The crude reaction mixture was pushed through a silica plug $(50 \%$ EtOAc/hexanes as the eluent) and concentrated in vacuo to afford the title compound as a white solid (2.3 g, 90\% yield). The spectral properties were consistent with the reported values. ${ }^{13}$ ${ }^{1}$ H NMR (600 MHz, Chloroform-d) $\delta 7.70(\mathrm{~d}, \mathrm{~J}=11.7 \mathrm{~Hz}, 2 \mathrm{H}), 7.62-7.53(\mathrm{~m}, 2 \mathrm{H}), 3.99$ $3.30(\mathrm{~m}, 8 \mathrm{H})$.<smiles>C/C=C/C(=O)NCCc1ccccc1</smiles>

(E)-N-phenethylbut-2-enamide (S15): To a flame dried round bottom flask was added crotonic acid ( 5 mmol, $0.43 \mathrm{~g}, 1$ equiv) after which the atmosphere was exchanged three times with nitrogen. $\mathrm{CH}_{2} \mathrm{Cl}_{2}(20 \mathrm{~mL})$ was then added followed by oxalyl chloride $(5.5 \mathrm{mmol}, 0.46 \mathrm{~mL}, 1.5$ equiv) and DMF (2 drops) - the reaction mixture was then allowed to stir for 1 hour.

Phenethylamine ( $6 \mathrm{mmol}, 0.76 \mathrm{~mL}, 1.2$ equiv) was then added in one portion followed by triethylamine $(5.5 \mathrm{mmol}, 0.77 \mathrm{~mL}, 1.1$ equiv). The reaction mixture was stirred overnight and was diluted with $\mathrm{CH}_{2} \mathrm{Cl}_{2}(20 \mathrm{~mL})$ and $1 \mathrm{M} \mathrm{HCl}(10 \mathrm{~mL})$ the next morning. The organic phase was separated from the aqueous phase and washed with $1 \mathrm{M} \mathrm{HCl}(10 \mathrm{ml})$, brine $(2 \mathrm{x})$, dried over $\mathrm{Na}_{2} \mathrm{SO}_{4}$, and concentrated in vacuo. The crude product was pushed through a silica plug with (50\% EtOAc/hexanes as the eluent) to afford the title compound as white solid (0.96 g, 99\% yield).

${ }^{1}$ H NMR (600 MHz, Chloroform-d) $\delta 7.31$ (t, J = 7.6 Hz, 1H), 7.24 (t, J = 7.9 Hz, 2H), 7.20 (d, $\mathrm{J}=7.3 \mathrm{~Hz}, 2 \mathrm{H}), 6.83(\mathrm{dq}, \mathrm{J}=15.2,6.9 \mathrm{~Hz}, 1 \mathrm{H}), 5.72(\mathrm{dd}, \mathrm{J}=15.2 \mathrm{~Hz}, 1.6 \mathrm{~Hz}, 1 \mathrm{H}), 5.39$ (bs, $1 \mathrm{H}), 3.59$ (td, J =6.9 Hz, $5.9 \mathrm{~Hz}, 2 \mathrm{H}), 2.84$ (t, J = 6.9 Hz, 2H), 1.83 (dd, J =6.9, $1.6 \mathrm{~Hz}, 3 \mathrm{H}$ ). ${ }^{13}$ C NMR (600 MHz, Chloroform-d) $\delta 165.9,140.5,138.5,128.8,128.7,125.9,124.9,40.53$, $35.7,17.2$.

HRMS (APCI) $m / z$ : [M+H] calcd. for $\mathrm{C}_{12} \mathrm{H}_{16} \mathrm{NO}$, 190.1226; found, 190.1229 . Rf: 0.34 (50\% EtOAc/Hexanes)

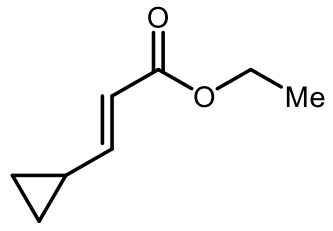

Ethyl $(\boldsymbol{E})$-3-cyclopropylacrylate (S16): The title compound was prepared according to literature procedure. The physical and spectral properties were consistent with the reported values. $^{14}$

${ }^{1}$ H NMR (600 MHz, Chloroform-d) $\delta 6.41(\mathrm{dd}, \mathrm{J}=15.4 \mathrm{~Hz}, 10.1 \mathrm{~Hz}, 1 \mathrm{H}), 5.89$ (d, J = $15.4 \mathrm{~Hz}$, $1 \mathrm{H}), 4.17(\mathrm{q}, \mathrm{J}=10.7 \mathrm{~Hz}, 2 \mathrm{H}), 1.58-1.53(\mathrm{~m}, 1 \mathrm{H}), 1.26(\mathrm{t}, \mathrm{J}=7.1 \mathrm{~Hz}, 3 \mathrm{H}), 0.95-0.91(\mathrm{~m}, 2 \mathrm{H})$, 0.65-0.61 (m, 2H). 
<smiles>O=C(/C=C/C1CC1)CCCc1ccccc1</smiles>

(E)-1-cyclopropyl-6-phenylhex-1-en-3-one (S17): To a flame dried round bottom flask was added 1-(triphenylphosphoraneylidene)-2-propanone (4 mmol, $1.27 \mathrm{~g}, 1$ equiv) after which the atmosphere was exchanged three times with nitrogen followed. THF was added $(30 \mathrm{~mL})$, the reaction mixture was cooled to $-78^{\circ} \mathrm{C}$ and n-butyllithium $(5.2 \mathrm{mmol}, 2.5 \mathrm{~mL}, 1.3$ equiv.) was added dropwise. The reaction mixture was stirred at $-78^{\circ} \mathrm{C}$ for 1 hour followed by the dropwise addition of (2-bromoethyl)benzene $(5.2 \mathrm{mmol}, 0.71 \mathrm{~mL}, 1.3$ equiv). The reaction mixture was warmed to room temperature, stirred for 7 hours, and concentrated in vacuo. The crude mixture was then dissolved in $\mathrm{CH}_{2} \mathrm{Cl}_{2}(40 \mathrm{~mL})$, washed with water (3x), dried over $\mathrm{Na}_{2} \mathrm{SO}_{4}$ and concentrated in vacuo. The crude reaction mixture was then dissolved in $\mathrm{CHCl}_{3}$ followed by the addition of cyclopropane carboxaldehyde ( $6 \mathrm{mmol}, 0.45 \mathrm{~mL}, 1.5$ equiv.). The reaction mixture was refluxed overnight at $60{ }^{\circ} \mathrm{C}$, concentrated in vacuo, and purified by silica chromatography $(0-5 \%$ EtOAc/hexanes as the eluent) to afford the title compound as a colorless oil $(0.35 \mathrm{~g}, 41 \%$ yield).

${ }^{1}$ H NMR (600 MHz, Chloroform-d) $\delta$ 7.28-7.22 (m, 2H), 7.19-7.13 (m, 3H), 6.27-6.14 (m, 2H), 2.61 (t, J = 7.6 Hz, 2H), $2.47(\mathrm{t}, \mathrm{J}=6.8 \mathrm{~Hz}, 2 \mathrm{H}), 1.96-1.88(\mathrm{~m}, 2 \mathrm{H}), 1.55-1.48(\mathrm{~m}, 1 \mathrm{H})$, 0.98-0.91 (m, 2H), 0.64-0.59 (m, 2H).

${ }^{13}$ C NMR (151 MHz, Chloroform-d) $\delta$ 199.65, 152.58, 141.92, 128.65, 128.49, 127.34, 126.03, 77.37, 77.16, 76.95, 39.59, 35.33, 25.95, 14.78, 9.08.

HRMS (APCI) $m / z$ : [M+H] calcd. for $\mathrm{C}_{15} \mathrm{H}_{19} \mathrm{O}, 215.1430$; found, 215.1233 .

Rf: 0.48 (10\% EtOAc/Hexanes)<smiles>COC(=O)/C=C/c1ccccn1</smiles>

Methyl (E)-3-(pyridine-2-yl)acrylate (S18): A round bottom flask was equipped with 2pyridinecarboxaldehyde ( $5 \mathrm{mmol}, 0.48 \mathrm{~mL}, 1$ equiv), methyl (triphenylphosphoranylidene)acetate $(7.5 \mathrm{mmol}, 2.50 \mathrm{~g}, 1.5 \mathrm{equiv})$ and benzene $(10 \mathrm{~mL})$ under nitrogen. The reaction was then heated to $80{ }^{\circ} \mathrm{C}$ for three hours, cooled to room temperature, and concentrated in vacuo. The crude product was purified by silica chromatography (30- 40\% EtOAc/hexanes as the eluent) to afford the title compound as a light brown solid $(0.59 \mathrm{~g}, 73 \%$ yield). The physical properties and spectral data were consistent with the reported values. ${ }^{15}$ ${ }^{1}$ H NMR (600 MHz, CDCl $)_{3} \delta 8.64(\mathrm{~d}, \mathrm{~J}=4.8 \mathrm{~Hz}, 1 \mathrm{H}), 7.73-7.64(\mathrm{~m}, 2 \mathrm{H}), 7.42$ (d, J = 7.8 Hz, 1H), 7.29-7.24 (m, 1H), $6.93(\mathrm{~d}, \mathrm{~J}=15.7 \mathrm{~Hz}, 1 \mathrm{H}), 3.81$ (s, 3H). 


\section{Preparation of Products from Substrate Table}

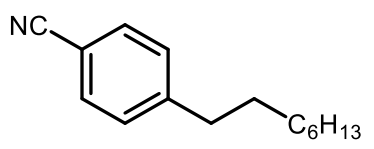

4-octylbenzonitrile (1): Prepared according to general procedure A using 4-chlorobenzonitrile ( $0.5 \mathrm{mmol}, 0.069 \mathrm{~g}, 1$ equiv.), 1 -octene $(2.5 \mathrm{mmol}, 390 \mu \mathrm{L}, 5.0$ equiv), sodium formate $(2.5$ mmol, $0.17 \mathrm{~g}, 5.0$ equiv), mesna ( $0.1 \mathrm{mmol}, 0.016 \mathrm{~g}, 20 \mathrm{~mol} \%)$ and 4CzIPN (0.005 mmol, $0.0039 \mathrm{~g}, 1 \mathrm{~mol} \%)$ in $50 \% \mathrm{MeCN} / \mathrm{DMSO}(5 \mathrm{~mL})$. After 16 hours the reaction was purified by silica chromatography (5\% EtOAC/hexanes as the eluent) to afford the title compound as a clear oil $(0.071 \mathrm{~g}, 66 \%$ yield $)$. The physical and spectral properties were consistent with the reported values. $^{16}$

${ }^{1}$ H NMR (500 MHz, Chloroform-d) $\delta 7.55(\mathrm{~d}, \mathrm{~J}=8.0 \mathrm{~Hz}, 2 \mathrm{H}), 7.25(\mathrm{~d}, \mathrm{~J}=8.0 \mathrm{~Hz}, 2 \mathrm{H}), 2.65$ (t, $\mathrm{J}=7.6 \mathrm{~Hz}, 2 \mathrm{H}), 1.60(\mathrm{~m}, 2 \mathrm{H}), 1.35-1.16(\mathrm{~m}, 10 \mathrm{H}), 0.87(\mathrm{t}, \mathrm{J}=6.8 \mathrm{~Hz}, 3 \mathrm{H})$.<smiles>N#Cc1ccc(CCCCO)cc1</smiles>

4-(4-hydroxybutyl)benzonitrile (2): Prepared according to general procedure A using 4chlorobenzonitrile ( $0.5 \mathrm{mmol}, 0.069 \mathrm{~g}, 1$ equiv.), 3-buten-1-ol (2.5 mmol, $215 \mu \mathrm{l}, 5$ equiv.), sodium formate $(2.5 \mathrm{mmol}, 0.17 \mathrm{~g}, 5.0$ equiv.), mesna $(0.1 \mathrm{mmol}, 0.016 \mathrm{~g}, 20 \mathrm{~mol} \%)$ and 4CzIPN (0.005 mmol, $0.0039 \mathrm{~g}, 1 \mathrm{~mol} \%$ ) in M 50\% MeCN/ DMSO (5 mL). After 16 hours, the crude product was purified by silica gel chromatography $(30 \%$ EtOAc/hexanes to $50 \%$

EtOAc:/Hexanes as the eluent) to afford the title compound as a colorless solid $(0.040 \mathrm{~g}, 46 \%$ yield).

${ }^{1}$ H NMR (500 MHz, Benzene-d6) $\delta 7.01(\mathrm{~d}, J=8.0 \mathrm{~Hz}, 2 \mathrm{H}), 6.56(\mathrm{~d}, J=8 . \mathrm{Hz}, 2 \mathrm{H}), 3.22(\mathrm{t}, \mathrm{J}=$ $6.3 \mathrm{~Hz}, 2 \mathrm{H}), 2.13$ (t, J = 7.6 Hz, 2H), 1.32-1.24 (m, 2H), 1.20-1.13 (m, 2H).

${ }^{13}$ C NMR (100 MHz, Chloroform-d) $\delta$ 148.17, 132.27, 129.31, 119.23, 109.71, 62.57, 35.88, 32.18, 27.23.

HRMS (APCI) $m / z$ : [M+H] calcd. for $\mathrm{C}_{11} \mathrm{H}_{14} \mathrm{NO}, 176.1069$; found, 176.1071 .

Rf: $0.23(30 \%$ EtOAc/Hexanes)<smiles>N#Cc1ccc(CCCCl)cc1</smiles>

4-(6-chlorohexyl)benzonitrile (3): Prepared according to general procedure A using 4chlorobenzonitrile ( $0.5 \mathrm{mmol}, 0.069 \mathrm{~g}, 1.0$ equiv), 6-chlorohex-1-ene ( $2.5 \mathrm{mmol}, 0.33 \mathrm{~mL}, 5.0$ equiv), sodium formate ( $2.5 \mathrm{mmol}, 0.17 \mathrm{~g}, 5.0$ equiv), mesna $(0.1 \mathrm{mmol}, 0.016 \mathrm{~g}, 20 \mathrm{~mol} \%)$ and 4CzIPN (0.005 mmol, $0.0039 \mathrm{~g}, 1 \mathrm{~mol} \%)$ in 50\% MeCN/ DMSO (5 mL). After 16 hours the reaction was purified by silica chromatography (0\%- 20\% EtOAc/hexanes as eluent) to afford the title compound as a clear oil (0.094 g, 85\% yield).

${ }^{1}$ H NMR (400 MHz, Chloroform-d) $\delta 7.57(\mathrm{~d}, \mathrm{~J}=8.3 \mathrm{~Hz}, 2 \mathrm{H}), 7.27(\mathrm{~d}, \mathrm{~J}=6.9 \mathrm{~Hz}, 2 \mathrm{H}), 3.53(\mathrm{t}$, $\mathrm{J}=6.6 \mathrm{~Hz}, 2 \mathrm{H}), 2.71-2.58(\mathrm{~m}, 2 \mathrm{H}), 1.82-1.70(\mathrm{~m}, 2 \mathrm{H}), 1.64(\mathrm{p}, \mathrm{J}=7.6 \mathrm{~Hz}, 2 \mathrm{H}), 1.54-1.41$ $(\mathrm{m}, 2 \mathrm{H}), 1.40-1.31(\mathrm{~m}, 2 \mathrm{H})$. 
${ }^{13}$ C NMR (101 MHz, Chloroform-d) $\delta$ 148.35, 132.44, 132.29, 129.31, 119.29, 109.73, 45.14, 36.07, 32.55, 30.89, 28.51, 26.74 .

HRMS (APCI) $m / z:[\mathrm{M}+\mathrm{H}]$ calcd. for $\mathrm{C}_{13} \mathrm{H}_{17} \mathrm{NCl}, 222.1044$; found, 222.1043 .

Rf: 0.40 (10\% EtOc/ Hexanes)<smiles>CC(C)C(c1ccc(C#N)cc1)[N+](=O)[O-]</smiles>

4-(3-methylbutan-2-yl)benzonitrile (4): Prepared according to general procedure A using 4chlorobenzonitrile ( $0.5 \mathrm{mmol}, 0.069 \mathrm{~g}, 1$ equiv.), 2-methyl-2-butene ( $2.5 \mathrm{mmol}, 265 \mu \mathrm{l}, 5$ equiv.), sodium formate ( $2.5 \mathrm{mmol}, 0.17 \mathrm{~g}, 5.0$ equiv), mesna $(0.1 \mathrm{mmol}, 0.016 \mathrm{~g}, 20 \mathrm{~mol} \%)$ and 4CzIPN (0.005 mmol, $0.0039 \mathrm{~g}, 1 \mathrm{~mol} \%)$ in 50\% MeCN/ DMSO (5 mL). After 16 hours, the crude product was purified by silica gel chromatography ( $5 \%$ EtOAc/hexanes as the eluent) to afford the title compound as a light yellow oil $(0.057 \mathrm{~g}, 66 \%$ yield $)$.

${ }^{1}$ H NMR (500 MHz, Benzene-d6) $\delta 7.01(\mathrm{~d}, J=8.0 \mathrm{~Hz}, 2 \mathrm{H}), 6.56(\mathrm{~d}, J=8.0 \mathrm{~Hz}, 2 \mathrm{H}), 1.99(\mathrm{q}, \mathrm{J}$ $=7.2 \mathrm{~Hz}, 1 \mathrm{H}), 1.43-1.31(\mathrm{~m}, 1 \mathrm{H}), 0.88(\mathrm{~d}, \mathrm{~J}=7.1 \mathrm{~Hz}, 3 \mathrm{H}), 0.71(\mathrm{~d}, \mathrm{~J}=6.7 \mathrm{~Hz}, 3 \mathrm{H}), 0.53(\mathrm{~d}, \mathrm{~J}=$ $6.7 \mathrm{~Hz}, 3 \mathrm{H})$.

${ }^{13}$ C NMR (100 MHz, Chloroform-d) $\delta$ 152.95, 132.11, 128.56, 119.35, 109.69, 47.24, 34.41, $36.62,21.17,20.16,18.60$.

HRMS (APCI) $m / z$ : [M+H] calcd. for $\mathrm{C}_{12} \mathrm{H}_{16} \mathrm{~N}, 174.1277$; found, 174.1277 .

Rf: 0.66 (5\% EtOAc/Hexanes)<smiles>CCOCCc1ccc(C#N)cc1</smiles>

4-(2-ethoxyethyl)benzonitrile (5): Prepared according to general procedure A using 4-chlorobenzontrile ( $0.5 \mathrm{mmol}, 0.07 \mathrm{~g}, 1.0$ equiv), ethyl vinyl ether $(2.5 \mathrm{mmol}, 0.24 \mathrm{~mL}, 5.0$ equiv), sodium formate $(2.5 \mathrm{mmol}, 0.17 \mathrm{~g}, 5.0$ equiv), mesna ( $0.1 \mathrm{mmol}, 0.016 \mathrm{~g}, 20 \mathrm{~mol} \%)$ and $4 \mathrm{CzIPN}$ $(0.005 \mathrm{mmol}, 0.0039 \mathrm{~g}, 1 \mathrm{~mol} \%)$ in $50 \% \mathrm{MeCN} / \mathrm{DMSO}(5 \mathrm{~mL})$. After 16 hours the reaction was purified by silica chromatography $(0 \%-50 \%$ EtOAc/hexanes as eluent) to provide the title compound as a clear oil $(0.070 \mathrm{~g}, 81 \%$ yield $)$

${ }^{1}$ H NMR (500 MHz, Chloroform-d) $\boldsymbol{\delta} 7.58(\mathrm{~d}, J=8.2 \mathrm{~Hz}, 2 \mathrm{H}), 7.34(\mathrm{~d}, J=8.1 \mathrm{~Hz}, 2 \mathrm{H}), 3.64$ (t, $J=6.7 \mathrm{~Hz}, 2 \mathrm{H}), 3.48$ (q, $J=7.0 \mathrm{~Hz}, 2 \mathrm{H}), 2.93$ (t, $J=6.7 \mathrm{~Hz}, 2 \mathrm{H}), 1.18(\mathrm{t}, J=7.0 \mathrm{~Hz}, 3 \mathrm{H})$.

${ }^{13}$ C NMR (151 MHz, Chloroform-d) $\delta 145.24,132.25,129.85,119.22,110.23,70.57,66.53$, $36.62,15.24$.

HRMS (APCI) $m / z$ : [M+H] calcd. for $\mathrm{C}_{11} \mathrm{H}_{14} \mathrm{NO}, 176.1069$; found, 176.1070 .

Rf: $0.25(10 \%$ EtOc/ hexanes $)$ 


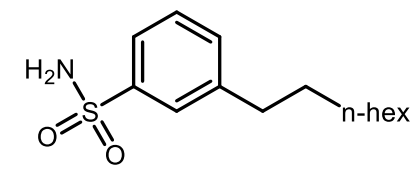

3-octylbenzenesulfonamide (6): Prepared according to general procedure A using 3bromobenzenesulfonamide ( $0.5 \mathrm{mmol}, 0.118 \mathrm{~g}, 1$ equiv. $), 1$-octene ( $2.5 \mathrm{mmol}, 390 \mu \mathrm{l}, 5$ equiv.), sodium formate $(2.5 \mathrm{mmol}, 0.17 \mathrm{~g}, 5.0$ equiv.), mesna ( $0.1 \mathrm{mmol}, 0.016 \mathrm{~g}, 20 \mathrm{~mol} \%)$ and 4CzIPN (0.005 mmol, $0.0039 \mathrm{~g}, 1 \mathrm{~mol} \%$ ) in 50\% MeCN/ DMSO (5 mL). After 16 hours, the crude product was purified by silica gel chromatography $(30 \%-50 \%$ EtOAc/hexanes as the eluent) to afford the title compound as a clear amorphous solid (0.068 g, 51\% yield).

${ }^{1}$ H NMR (500 MHz, Chloroform-d) $\delta$ 7.76-7.72 (m, 2H), 7.44-7.37 (m, 2H), $4.75(\mathrm{~s}, 2 \mathrm{H}), 2.67$ $(\mathrm{t}, \mathrm{J}=7.8 \mathrm{~Hz}, 2 \mathrm{H}), 1.66-1.58(\mathrm{~m}, 2 \mathrm{H}), 1.36-1.21(\mathrm{~m}, 10 \mathrm{H}), 0.88 \mathrm{f}(\mathrm{t}, \mathrm{J}=6.7 \mathrm{~Hz}, 3 \mathrm{H})$.

${ }^{13}$ C NMR (101 MHz, Chloroform-d) $\delta$ 144.60, 141.91, 133.03, 129.15, 126.24, 123.80, 35.89, 31.97, 31.34, 29.51, 29.37, 29.33, 22.78, 14.23.

HRMS (APCI) $m / z$ : [M+H] calcd. for $\mathrm{C}_{14} \mathrm{H}_{24} \mathrm{NO}_{3} \mathrm{~S}, 270.1522$; found, 270.1515 .

Rf: 0.75 (40\% EtOAc/Hexanes)<smiles>CCCCCNCCc1cccc(P(=O)(OCC)OCC)c1</smiles>

Diethyl (4-octlyphenyl)phosphonate (7): Prepared according to general procedure A using diethyl (3-bromophenyl)phosphonate $\mathbf{S 3}$ (0.5 mmol, $0.147 \mathrm{~g}$, 1 equiv.), 1-octene (2.5 mmol, 390 $\mu \mathrm{l}, 5$ equiv.), sodium formate $(2.5 \mathrm{mmol}, 0.17 \mathrm{~g}, 5.0$ equiv.), mesna $(0.1 \mathrm{mmol}, 0.016 \mathrm{~g}, 20$ mol\%) and 4CzIPN (0.005 mmol, $0.0039 \mathrm{~g}, 1 \mathrm{~mol} \%)$ in 50\% MeCN/ DMSO (5 mL). After 16 hours, the crude product was purified by silica gel chromatography (15\% EtOAc/hexanes to 50\% EtOAc/hexanes as the eluent) to afford the title compound as a colorless oil ( $0.060 \mathrm{~g}, 37 \%$ yield). ${ }^{1}$ H NMR (500 MHz, Chloroform-d) $\delta$ 7.65-7.57 (m, 2H), 7.38-7.34 (m, 2H), 4.19-4.02 (m, $2 \mathrm{H}), 2.64(\mathrm{t}, \mathrm{J}=7.7 \mathrm{~Hz}, 2 \mathrm{H}), 1.65-1.58(\mathrm{~m}, 2 \mathrm{H}), 1.35-1.22(\mathrm{~m}, 10 \mathrm{H}), 0.87(\mathrm{t}, \mathrm{J}=12.9 \mathrm{~Hz}, 3 \mathrm{H})$.

${ }^{13}$ C NMR (151 MHz, Chloroform-d) $\delta 143.46(\mathrm{~d}, J=14.5 \mathrm{~Hz}), 132.67(\mathrm{~d}, J=3.2 \mathrm{~Hz}), 131.89$ $(\mathrm{d}, J=10.2 \mathrm{~Hz}), 129.13(\mathrm{~d}, J=9.8 \mathrm{~Hz}), 128.51(\mathrm{~d}, J=15.6 \mathrm{~Hz}), 128.21(\mathrm{~d}, J=186.5 \mathrm{~Hz}), 62.16$ $(\mathrm{d}, J=5.4 \mathrm{~Hz}), 35.92,31.98,31.46,29.53,29.39,29.36,22.78,16.46$ (d, $J=6.5 \mathrm{~Hz}), 14.21$.

${ }^{31}$ P NMR (243 MHz, Chloroform-d) $\delta 19.42$

HRMS (APCI) $m / z$ : [M+H] calcd. for $\mathrm{C}_{18} \mathrm{H}_{32} \mathrm{O}_{3} \mathrm{P}, 327.2084$; found, 327.2078 .

Rf: $0.62(50 \%$ EtOAc/Hexanes)<smiles>CC(=O)NCCc1cccc(C(C)=O)c1</smiles>

Methyl 3-octylbenzoate (8): Prepared according to general procedure A using methyl 3chlorobenzoate $(0.5 \mathrm{mmol}, 70 \mu \mathrm{L}, 1.0$ equiv $), 1$-octene $(2.5 \mathrm{mmol}, 390 \mu \mathrm{L}, 5.0$ equiv $)$, sodium formate ( $2.5 \mathrm{mmol}, 0.17 \mathrm{~g}, 5.0$ equiv), mesna ( $0.1 \mathrm{mmol}, 0.016 \mathrm{~g}, 20 \mathrm{~mol} \%)$ and 4CzIPN (0.005 mmol, $0.0039 \mathrm{~g}, 1 \mathrm{~mol} \%)$ in 50\% MeCN/ DMSO (5 mL). After 16 hours, the crude product was pushed through a silica plug using EtOAc, concentrated in vacuo, and left under vacuum 
overnight to afford the title compound as a colorless oil $(0.086 \mathrm{~g}, 69 \%$ yield $)$. The physical and spectral properties were consistent with the reported values. ${ }^{17}$

${ }^{1}$ H NMR (500 MHz, Chloroform-d) $\delta$ 7.88-7.82 (m, 2H), 7.39-7.31 (m, 2H), $3.91(\mathrm{~s}, 3 \mathrm{H}), 2.65$ $(\mathrm{t}, \mathrm{J}=7.9 \mathrm{~Hz} 2 \mathrm{H}), 1.61(\mathrm{~m}, 2 \mathrm{H}), 1.36-1.21(\mathrm{~m}, 10 \mathrm{H}), 0.88(\mathrm{t}, \mathrm{J}=6.9 \mathrm{~Hz}, 3 \mathrm{H})$.

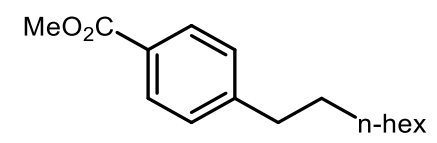

Methyl-4-octylbenzoate (9): Prepared according to general procedure A using methyl 4chlorobenzoate ( $0.5 \mathrm{mmol}, 0.085 \mathrm{~g}, 1.0$ equiv), 1 -octene $(2.5 \mathrm{mmol}, 390 \mu \mathrm{L}, 5.0$ equiv), sodium formate ( $2.5 \mathrm{mmol}, 0.17 \mathrm{~g}, 5.0$ equiv), mesna ( $0.1 \mathrm{mmol}, 0.016 \mathrm{~g}, 20 \mathrm{~mol} \%)$ and 4CzIPN (0.005 mmol, $0.0039 \mathrm{~g}, 1 \mathrm{~mol} \%$ ) in 50\% MeCN/ DMSO (5 mL). After 16 hours, the crude product was pushed through a silica plug using EtOAc, concentrated in vacuo, and left under vacuum overnight to afford the title compound as a light yellow oil $(0.093 \mathrm{~g}, 75 \%$ yield $)$. The physical and spectral properties were consistent with the reported values. ${ }^{18}$

${ }^{1}$ H NMR (500 MHz, Chloroform-d) $\delta 7.95(\mathrm{~d}, \mathrm{~J}=8.0 \mathrm{~Hz}, 2 \mathrm{H}), 7.24(\mathrm{~d}, \mathrm{~J}=8.0 \mathrm{~Hz}, 2 \mathrm{H}), 3.90$ (s, 3H), 2.65 (t, J = 7.8 Hz 2H), 1.64-1.59 (m, 2H), 1.32-1.22 (m, 10H), 0.89 (t, J = 7.0 Hz, 3H).

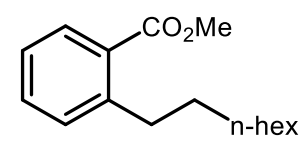

Methyl-2-octylbenzoate (10): Prepared according to general procedure A using methyl 2chlorobenzoate ( $0.5 \mathrm{mmol}, 72 \mu \mathrm{l}, 1$ equiv.), 1-octene $(2.5 \mathrm{mmol}, 390 \mu \mathrm{l}, 5$ equiv.), sodium formate ( $2.5 \mathrm{mmol}, 0.17 \mathrm{~g}, 5.0$ equiv.), mesna ( $0.1 \mathrm{mmol}, 0.016 \mathrm{~g}, 20 \mathrm{~mol} \%)$ and 4CzIPN (0.005 $\mathrm{mmol}, 0.0039 \mathrm{~g}, 1 \mathrm{~mol} \%$ ) in 50\% MeCN/ DMSO (5 mL). After 16 hours, the crude product was purified by silica gel chromatography (5\% EtOAc/hexanes as the eluent) to afford the title compound as a colorless oil $(0.040 \mathrm{~g}, 32 \%$ yield $)$.

${ }^{1}$ H NMR (500 MHz, Chloroform-d) $\delta 7.84(\mathrm{dd}, \mathrm{J}=7.8,1.5 \mathrm{~Hz}, 2 \mathrm{H}), 7.41(\mathrm{td}, \mathrm{J}=7.5,1.3 \mathrm{~Hz}$, $2 \mathrm{H}), 3.89$ (s, 3H), 2.93 (t, J = 7.8 Hz, 2H), 1.62-1.54 (m, 2H), 1.36-1.21 (m, 10H), $0.87(\mathrm{t}, \mathrm{J}=$ $7.04 \mathrm{~Hz}, 3 \mathrm{H})$.

${ }^{13}$ C NMR (151 MHz, Chloroform-d) $\delta 168.41,144.87,131.91,131.03,130.66,129.66,125.77$, 52.00, 34.61, 32.04, 31.98, 29.91, 29.63, 29.43, 22.82, 14.25 .

HRMS (APCI) $m / z$ : [M+H] calcd. for $\mathrm{C}_{16} \mathrm{H}_{25} \mathrm{O}_{2}, 249.1849$; found, 249.1851.

Rf: 0.59 (5\% EtOAc/Hexanes).

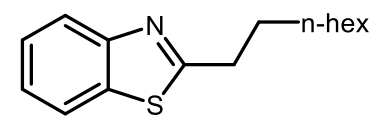

2-octylbenzo $[\boldsymbol{d}]$ thioazole (11): Prepared according to general procedure A at $100{ }^{\circ} \mathrm{C}$ using 2bromobenzothiazole ( $0.5 \mathrm{mmol}, 0.107 \mathrm{~g}, 1.0$ equiv), 1 -octene $(2.5 \mathrm{mmol}, 390 \mu \mathrm{L}, 5.0$ equiv), sodium formate $(2.5 \mathrm{mmol}, 0.17 \mathrm{~g}, 5.0$ equiv), mesna $(0.1 \mathrm{mmol}, 0.016 \mathrm{~g}, 20 \mathrm{~mol} \%)$ and $4 \mathrm{CzIPN}$ $(0.005 \mathrm{mmol}, 0.0039 \mathrm{~g}, 1 \mathrm{~mol} \%)$ in $50 \% \mathrm{MeCN} / \mathrm{DMSO}(5 \mathrm{~mL})$. After 16 hours the reaction was purified by silica chromatography (5\% EtOAc/hexanes as the eluent) to afford the title compound as a yellow oil (0.099 g, 80\% yield). The physical and spectral properties were 
consistent with the reported values. ${ }^{19}$

${ }^{1}$ H NMR (500 MHz, Chloroform-d) $\delta 7.97(\mathrm{dd}, \mathrm{J}=8.2,1.2 \mathrm{~Hz}, 1 \mathrm{H}), 7.83(\mathrm{~d}, \mathrm{~J}=8.0,1.3 \mathrm{~Hz}$, 1H), 7.44 (dt, J = 8.3, 1.3 Hz 1H), 7.33 (dt, J = 7.4, $1.2 \mathrm{~Hz} 1 \mathrm{H}), 3.11(\mathrm{t}, \mathrm{J}=7.4 \mathrm{~Hz}, 2 \mathrm{H}), 1.46-$ $1.24(\mathrm{~m}, 12 \mathrm{H}), 0.88(\mathrm{t}, \mathrm{J}=6.9 \mathrm{~Hz}, 3 \mathrm{H})$.

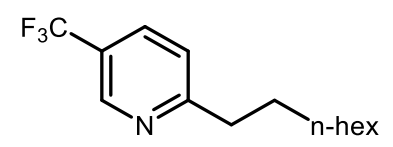

2-octyl-5-(trifluoromethyl)pyridine (12): Prepared according to general procedure A using 2chloro-5-(trifluoromethyl)pyridine $(0.5 \mathrm{mmol}, 0.091 \mathrm{~g}, 1.0$ equiv), 1 -octene $(2.5 \mathrm{mmol}, 0.39 \mathrm{~mL}$, 5.0 equiv), sodium formate $(2.5 \mathrm{mmol}, 0.17 \mathrm{~g}, 5.0$ equiv), mesna $(0.1 \mathrm{mmol}, 0.016 \mathrm{~g}, 20 \mathrm{~mol} \%)$ and 4CzIPN (0.005 mmol, $0.0039 \mathrm{~g}, 1 \mathrm{~mol} \%)$ in 50\% MeCN/ DMSO (5 mL). After 16 hours the reaction was purified by silica chromatography $(0 \%-50 \%$ EtOAc/hexanes as the eluent) to provide the title compound as a clear oil $(0.09 \mathrm{~g}, 70 \%$ yield). The spectral properties were consistent with the reported values. ${ }^{20}$

${ }^{1}$ H NMR (400 MHz, Chloroform-d) $\delta 8.81(\mathrm{~d}, J=2.4 \mathrm{~Hz}, 1 \mathrm{H}), 7.84(\mathrm{dd}, J=8.2,2.5 \mathrm{~Hz}, 1 \mathrm{H})$, $7.29(\mathrm{~d}, J=8.2 \mathrm{~Hz}, 1 \mathrm{H}), 2.92-2.78(\mathrm{~m}, 2 \mathrm{H}), 1.76(\mathrm{p}, J=7.8 \mathrm{~Hz}, 2 \mathrm{H}), 1.41-1.18(\mathrm{~m}, 10 \mathrm{H})$, $0.96-0.84(\mathrm{~m}, 3 \mathrm{H})$.<smiles>O=C(O)CCc1ccccn1</smiles>

2-octylpyridine (13): Prepared according to general procedure A using 2-chloro-pyridine $(0.25$ mmol, $24 \mu \mathrm{L}, 1.0$ equiv), 1-octene ( $1.25 \mathrm{mmol}, 0.20 \mathrm{~mL}, 5.0$ equiv), sodium formate (1.25 mmol, $0.085 \mathrm{~g}, 5.0$ equiv), formic acid ( $1.25 \mathrm{mmol}, 47 \mu \mathrm{L}, 5.0$ equiv), mesna ( $0.05 \mathrm{mmol}$, $0.0082 \mathrm{~g}, 20 \mathrm{~mol} \%)$ and 4CzIPN (0.0025 mmol, $0.0020 \mathrm{~g}, 1 \mathrm{~mol} \%)$ in 50\% MeCN/ DMSO (2.5 $\mathrm{mL})$. After 16 hours the reaction was purified quenched with $\mathrm{NaHCO}_{3}$ and extracted with EtOAc (3x). It was purified by silica chromatography $(0 \%-50 \%$ EtOAc/hexanes as the eluent) to provide the title compound as a pale yellow oil $(0.020 \mathrm{~g}, 43 \%$ yield $)$. The spectral properties were consistent with the reported values. ${ }^{21}$

${ }^{1}$ H NMR (600 MHz, Chloroform-d) $\delta 8.70-8.26(\mathrm{~m}, 1 \mathrm{H}), 7.58(\mathrm{td}, J=7.6,1.9 \mathrm{~Hz}, 1 \mathrm{H}), 7.14$ $(\mathrm{d}, J=7.8 \mathrm{~Hz}, 1 \mathrm{H}), 7.09$ (ddd, $J=7.6,4.9,1.2 \mathrm{~Hz}, 1 \mathrm{H}), 2.81-2.75$ (m, 2H), $1.76-1.68$ (m, $2 \mathrm{H}), 1.41-1.19(\mathrm{~m}, 10 \mathrm{H}), 0.87(\mathrm{t}, J=7.0 \mathrm{~Hz}, 3 \mathrm{H})$.

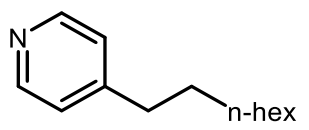

4-octylpyridine (14): Prepared according to general procedure A using 4-chloropyridin-1-ium chloride ( $0.5 \mathrm{mmol}, 0.075 \mathrm{~g}, 1.0$ equiv), 1 -octene $(2.5 \mathrm{mmol}, 0.39 \mathrm{~mL}, 5.0$ equiv), sodium formate (2.5 mmol, $0.17 \mathrm{~g}, 5.0$ equiv), mesna ( $0.1 \mathrm{mmol}, 0.016 \mathrm{~g}, 20 \mathrm{~mol} \%)$ and $4 \mathrm{CzIPN}(0.005$ $\mathrm{mmol}, 0.0039 \mathrm{~g}, 1 \mathrm{~mol} \%)$ in 50\% MeCN/ DMSO (5 mL). After 16 hours the reaction was purified quenched with $\mathrm{NaHCO}_{3}$ and extracted with EtOAc (3x). It was purified by silica 
chromatography (10\%- 50\% EtOAc/hexanes as eluent) to provide the title compound as a yellow oil $(0.056 \mathrm{~g}, 70 \%$ yield $)$. The spectral properties were consistent with the reported values. ${ }^{22}$

${ }^{1}$ H NMR (500 MHz, Chloroform-d) $\delta 8.48(\mathrm{~d}, J=5.2 \mathrm{~Hz}, 2 \mathrm{H}), 7.10(\mathrm{~d}, J=4.4 \mathrm{~Hz}, 2 \mathrm{H}), 2.63$ $2.56(\mathrm{~m}, 2 \mathrm{H}), 1.62(\mathrm{p}, J=7.3 \mathrm{~Hz}, 2 \mathrm{H}), 1.48-1.12(\mathrm{~m}, 10 \mathrm{H}), 0.88(\mathrm{t}, J=6.9 \mathrm{~Hz}, 3 \mathrm{H})$.

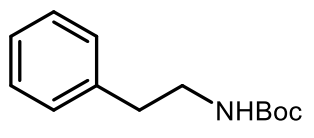

Tert-butyl phenethylcarbamate (15): Prepared according to general procedure A using bromobenzene ( $0.5 \mathrm{mmol}, 53 \mu \mathrm{L}, 1.0$ equiv), tert-butyl vinylcarbamate $\mathbf{S 2}$ (1.5 mmol, $0.214 \mathrm{~g}$, 3.0 equiv), sodium formate $(2.5 \mathrm{mmol}, 0.17 \mathrm{~g}, 5.0$ equiv), mesna $(0.1 \mathrm{mmol}, 0.016 \mathrm{~g}, 20 \mathrm{~mol} \%)$ and $4 \mathrm{CzIPN}(0.005 \mathrm{mmol}, 0.0039 \mathrm{~g}, 1 \mathrm{~mol} \%)$ in DMSO $(5 \mathrm{~mL})$. After 16 hours the reaction was purified by silica chromatography $(10 \%-15 \%$ EtOAc/Hexanes as the eluent) to afford the title compound as a white solid (0.082 $\mathrm{g}, 74 \%$ yield). The spectral properties were consistent with the reported values. ${ }^{23}$

${ }^{1}$ H NMR (500 MHz, Chloroform-d) $\delta$ 7.32-7.28 (m, 2H), 7.24-7.17 (m, 3H), 3.42-2.32 (m, 2H), $2.80(\mathrm{t}, \mathrm{J}=7.0 \mathrm{~Hz}, 2 \mathrm{H}), 1.43(\mathrm{~s}, 9 \mathrm{H})$.

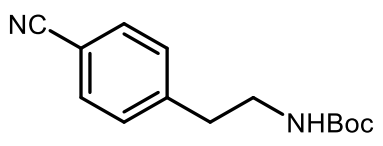

Tert-butyl (4-cyanophenethyl)carbamate (16): Prepared according to general procedure A using 4-chlorobenzonitrile ( $0.5 \mathrm{mmol}, 0.069 \mathrm{~g}, 1$ equiv), tert-butyl vinylcarbamate $\mathbf{S 2}$ ( $1.5 \mathrm{mmol}$, $0.214 \mathrm{~g}, 3.0$ equiv), sodium formate $(2.5 \mathrm{mmol}, 0.17 \mathrm{~g}, 5.0$ equiv), mesna ( $0.1 \mathrm{mmol}, 0.016 \mathrm{~g}, 20$ mol\%) and 4CzIPN (0.005 mmol, $0.0039 \mathrm{~g}, 1 \mathrm{~mol} \%)$ in DMSO $(5 \mathrm{~mL})$. The crude product was purified on silica gel (20\%-30\% EtOAc/Hexanes as the eluent) to afford the title compound as a white solid ( $0.100 \mathrm{~g}, 81 \%$ yield). The physical and spectral properties were consistent with the reported values. ${ }^{24}$

${ }^{1}$ H NMR (500 MHz, Chloroform-d): $\delta 7.59$ (d, J = 7.8 Hz 2H), 7.65-7.57 (m, 2H), 4.53 (br s, 1H) $3.43-3.34(\mathrm{~m}, 2 \mathrm{H}), 2.89-2.83(\mathrm{~m}, 2 \mathrm{H}), 1.42(\mathrm{~m}, 9 \mathrm{H})$.

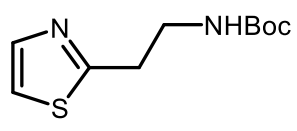

Tert-butyl (2-(thiazol-2-yl)ethyl) carbamate (17): Prepared according to general procedure A using 2-bromothiazole ( $0.5 \mathrm{mmol}, 45 \mu \mathrm{l}, 1$ equiv), tert-butyl vinylcarbamate $\mathbf{S 2}$ (1.5 mmol, 0.214 $\mathrm{g}, 1$ equiv), sodium formate $(2.5 \mathrm{mmol}, 0.17 \mathrm{~g}, 5.0$ equiv. $)$, mesna $(0.1 \mathrm{mmol}, 0.016 \mathrm{~g}, 20 \mathrm{~mol} \%)$ and 4CzIPN (0.005 mmol, $0.0039 \mathrm{~g}, 1 \mathrm{~mol} \%)$ in DMSO $(5 \mathrm{~mL})$. After 16 hours, the crude product was purified by silica gel chromatography (20\%- 50\% EtOAc/Hexanes as the eluent) to afford the title compound as a yellow solid $(0.112 \mathrm{~g}, 98 \%$ yield $)$.

${ }^{1}$ H NMR (500 MHz, Chloroform-d) $\delta 7.70(\mathrm{~d}, J=3.4 \mathrm{~Hz}, 1 \mathrm{H}), 7.23$ (d, $\left.J=3.3 \mathrm{~Hz}, 1 \mathrm{H}\right), 5.12$ 
(s, 1H), 3.62-3.53 (m, 2H), $3.22(\mathrm{t}, \mathrm{J}=6.4 \mathrm{~Hz}, 2 \mathrm{H}), 1.44(\mathrm{~s}, 9 \mathrm{H})$.

${ }^{13}$ C NMR (100 MHz, Chloroform-d) $\delta 168.11,155.93,142.56,118.71,79.43,39.92,33.41$, 28.49 .

HRMS (APCI) m/z: [M+H] calcd. for $\mathrm{C}_{10} \mathrm{H}_{17} \mathrm{~N}_{2} \mathrm{O}_{2}, 229.1005$; found, 229.1007.

Rf: 0.50 (40\% EtOAc/Hexanes)<smiles>N#Cc1ccccc1</smiles>

Benzonitrile (18): Prepared according to general procedure B using 3-cyano-N,N,Ntrimethylbenzenaminium iodide $\mathbf{S 5}(0.25 \mathrm{mmol}, 0.072 \mathrm{~g}, 1.0$ equiv $)$, sodium formate $(1.25$ mmol, $0.085 \mathrm{~g}, 5.0$ equiv), mesna (0.05 mmol, $0.0082 \mathrm{~g}, 20 \mathrm{~mol} \%)$ and 4CzIPN (0.0025 mmol, $0.0020 \mathrm{~g}, 1 \mathrm{~mol} \%)$ in $\mathrm{d}_{6}$-DMSO $(2.5 \mathrm{~mL})$. After 16 hours the reaction was stopped and dibromomethane was added $(0.25 \mathrm{mmol}, 17.5 \mu \mathrm{L}, 1.0$ equiv $)$ as internal standard. The sample was analyzed by ${ }^{1} \mathrm{H}$ NMR $(\mathrm{d}=5 \mathrm{~s})$, and the integral values were used to calculate the product yield ( $86 \%$ yield by NMR).<smiles>CCOP(=O)(OCC)c1ccccc1</smiles>

diethyl phenylphosphonate (19): Prepared according to general procedure B using 3(diethoxyphosphoryl)-N,N,N-trimethylbenzenaminium iodide $\mathbf{S 7}$ (0.25 mmol, $0.099 \mathrm{~g}, 1.0$ equiv), sodium formate ( $1.25 \mathrm{mmol}, 0.085 \mathrm{~g}, 5.0$ equiv), mesna ( $0.05 \mathrm{mmol}, 0.0082 \mathrm{~g}, 20 \mathrm{~mol} \%)$ and 4CzIPN (0.0025 mmol, $0.0020 \mathrm{~g}, 1 \mathrm{~mol} \%)$ in DMSO (2.5 mL). After 16 hours the reaction was washed with water and extracted with EtOAc $(3 \mathrm{x})$ to provide the title compound with no extra purification needed as a tan oil $(0.050 \mathrm{~g}, 94 \%$ yield $)$. The spectral properties were consistent with the reported values. ${ }^{25}$

${ }^{1}$ H NMR (600 MHz, Chloroform-d) $\delta 7.81$ (ddd, J = 13.3, 8.3, 1.4 Hz, 2H), $7.61-7.51$ (m, $1 \mathrm{H}), 7.46$ (td, J = 7.5, 4.3 Hz, 2H), $4.19-4.02(\mathrm{~m}, 4 \mathrm{H}), 1.32(\mathrm{t}, \mathrm{J}=6.8 \mathrm{~Hz}, 6 \mathrm{H})$.<smiles>CC(=O)c1ccccc1</smiles>

Methylbenzoate (20): Prepared according to general procedure B using 3-(methoxycarbonyl)N,N,N-trimethylbenzenaminium iodide $\mathbf{S 9}(0.5 \mathrm{mmol}, 0.16 \mathrm{~g}, 1.0$ equiv), sodium formate $(2.5$ mmol, $0.17 \mathrm{~g}, 5.0$ equiv), mesna ( $0.1 \mathrm{mmol}, 0.016 \mathrm{~g}, 20 \mathrm{~mol} \%)$ and $4 \mathrm{CzIPN}$ (0.005 mmol, $0.0039 \mathrm{~g}, 1 \mathrm{~mol} \%)$ in in DMSO $(5 \mathrm{~mL})$. After 16 hours the reaction was washed with water and extracted with EtOAc $(3 \mathrm{x})$ to provide the title compound with no extra purification needed as a yellow oil ( $0.047 \mathrm{~g}, 70 \%$ yield). The spectral properties were consistent with the reported values. $^{26}$

${ }^{1}$ H NMR (600 MHz, Chloroform-d) $\delta 8.04(\mathrm{~d}, J=7.1 \mathrm{~Hz}, 2 \mathrm{H}), 7.56(\mathrm{t}, J=7.4 \mathrm{~Hz}, 1 \mathrm{H}), 7.47$ $7.42(\mathrm{~m}, 2 \mathrm{H}), 3.92(\mathrm{~s}, 3 \mathrm{H})$. 
<smiles>c1ccc(Nc2ccccc2)cc1</smiles>

diphenyl amine (21): Prepared according to general procedure B at $100{ }^{\circ} \mathrm{C}$ using 4-methyl-N,Ndiphenylbenzenesulfonamide $\mathbf{S 1 0}$ ( $0.5 \mathrm{mmol}, 0.162 \mathrm{~g}, 1.0$ equiv), sodium formate $(5.0 \mathrm{mmol}$, $0.34 \mathrm{~g}, 5.0$ equiv), mesna ( $0.1 \mathrm{mmol}, 0.0164 \mathrm{~g}, 20 \mathrm{~mol} \%)$ and 4CzIPN (0.0039 mmol, $0.0039 \mathrm{~g}$, $1 \mathrm{~mol} \%$ ) in $20 \% \mathrm{H}_{2} \mathrm{O} / \mathrm{DMSO}(5 \mathrm{~mL})$. After 16 hours the reaction was purified by silica chromatography (30\%- 100\% EtOAc/hexanes as eluent) to provide the title compound as an off white solid $(0.044 \mathrm{~g}, 52 \%$ yield $)$. The spectral properties were consistent with the reported values. $^{27}$

${ }^{1}$ H NMR (600 MHz, Chloroform-d) $\delta 7.28(\mathrm{~d}, J=7.3 \mathrm{~Hz}, 4 \mathrm{H}), 7.08$ (d, J= 7.4 Hz, 4H), 6.93 (t, $J=7.4 \mathrm{~Hz}, 2 \mathrm{H}), 5.71($ br s, $1 \mathrm{H})$<smiles>Nc1cccnc1</smiles>

3-aminopyridine (22): Prepared according to general procedure B at $100{ }^{\circ} \mathrm{C}$ using 4-methyl- $N$ (pyridine-3-yl)benzenesulfonamide S11 ( $0.25 \mathrm{mmol}, 0.062 \mathrm{~g}, 1.0$ equiv), sodium formate $(2.5$ mmol, $0.17 \mathrm{~g}, 10.0$ equiv), mesna ( $0.05 \mathrm{mmol}, 0.0082 \mathrm{~g}, 20 \mathrm{~mol} \%)$ and 4CzIPN (0.0025 mmol, $0.0020 \mathrm{~g}, 1 \mathrm{~mol} \%)$ in $\mathrm{d}_{6}$-DMSO ( $\left.2.5 \mathrm{~mL}\right)$. After 16 hours the reaction was stopped and dibromomethane was added $(0.25 \mathrm{mmol}, 17.5 \mu \mathrm{L}, 1.0$ equiv $)$ as internal standard. The sample was analyzed by ${ }^{1} \mathrm{H}$ NMR $(\mathrm{d}=5 \mathrm{~s})$, and the integral values were used to calculate the product yield ( $84 \%$ yield by NMR).<smiles>c1ccc2[nH]cnc2c1</smiles>

1H-benzo[d]imidazole (23): Prepared according to general procedure B using 1-tosyl- $1 \mathrm{H}$ benzo[d] imidazole S12 ( $0.25 \mathrm{mmol}, 0.068 \mathrm{~g}, 1.0$ equiv), sodium formate (1.25 mmol, $0.085 \mathrm{~g}$, 5.0 equiv), mesna (0.05 mmol, $0.0082 \mathrm{~g}, 20 \mathrm{~mol} \%)$ and $4 \mathrm{CzIPN}(0.0025 \mathrm{mmol}, 0.0020 \mathrm{~g}, 1$ $\mathrm{mol} \%)$ in $20 \% \mathrm{H}_{2} \mathrm{O} / \mathrm{DMSO}(2.5 \mathrm{~mL})$. After 16 hours the reaction was purified by silica chromatography (30\%-100\% EtOAc/hexanes as eluent) to provide the title compound as an off white solid ( $0.024 \mathrm{~g}, 81 \%$ yield). The spectral properties were consistent with the reported values. $^{28}$

${ }^{1}$ H NMR (600 MHz, Chloroform-d) $\delta 8.14$ (s, 1H), 7.69 (br s, 2H), 7.31 (dd, J = 6.1, $3.0 \mathrm{~Hz}$, $2 \mathrm{H})$. 
<smiles>O=C(c1cccc(C(F)(F)CCCCO)c1)N1CCOCC1</smiles>

(3-(1,1-difluoro-5-hydroxypentyl)phenyl)(morpholino)methanone (24): Prepared according to general procedure A using morpholino(3-(trifluoromethyl)phenyl)methanone S14 (0.5 mmol, $0.129 \mathrm{~g}, 1$ equiv), 3-buten-1-ol ( $1.5 \mathrm{mmol}, 130 \mu \mathrm{L}, 3$ equiv), sodium formate $(2.5 \mathrm{mmol}, 0.17 \mathrm{~g}$, 5.0 equiv), mesna (0.1 mmol, $0.016 \mathrm{~g}, 20 \mathrm{~mol} \%)$ and $4 \mathrm{CzIPN}(0.005 \mathrm{mmol}, 0.0039 \mathrm{~g}, 1 \mathrm{~mol} \%)$ in in DMSO $(5 \mathrm{~mL})$. The crude product was purified by silica chromatography $(20 \%-30 \%$ EtOAc/hexanes as the eluent) to afford the title compound as a claer oil ( $0.078 \mathrm{~g}, 56 \%$ yield). The physical and spectral properties were consistent with the reported values. ${ }^{13}$

${ }^{1}$ H NMR (600 MHz, Chloroform-d): $\delta$ 7.55-7.43 (m, 4H), 3.78 (bs, 4H), 3.66-3.54 (m, 4H), 3.42 (bs, 2H), 2.23-2.04 (m, 2H), 1.73 (bs, 1H), 1.61-1.47 (m, 4H).<smiles>OCCCCC(F)(F)c1cccc(C(F)(F)F)c1</smiles>

5,5-difluoro-5-(3-(trifluoromethyl)phenyl)pentan-1-ol (25): Prepared according to general procedure A using 1,3-bis(trifluoromethyl)benzene $(0.5 \mathrm{mmol}, 78 \mu \mathrm{L}, 1.0$ equiv), 3-buten-1-ol ( $1.5 \mathrm{mmol}, 130 \mu \mathrm{L}, 3$ equiv), sodium formate $(2.5 \mathrm{mmol}, 0.17 \mathrm{~g}, 5.0$ equiv), mesna ( $0.1 \mathrm{mmol}$, $0.016 \mathrm{~g}, 20 \mathrm{~mol} \%)$ and 4CzIPN (0.005 mmol, $0.0039 \mathrm{~g}, 1 \mathrm{~mol} \%)$ in in DMSO (5 mL). The crude product was purified by silica chromatography (20\%-40\% EtOAc/hexanes as the eluent) to afford the title compound as a light yellow oil $(0.073 \mathrm{~g}, 56 \%$ yield $)$. The physical and spectral properties were consistent with the reported values. ${ }^{13}$

${ }^{1}$ H NMR (600 MHz, Chloroform-d): $\delta 7.78$ (d, J = $\left.7.9 \mathrm{~Hz} 2 \mathrm{H}\right), 7.30$ (d, J = 7.9 Hz, 2H), 4.54 (br s, 1H), 3.42-3.33 (m, 2H), 2.89-2.82 (m, 2H), 1.42 (s, 9H).<smiles>OCCCCC(F)(F)c1ccccn1</smiles>

4,4-difluoro-4-(pyridine-2-yl)butan-1-ol (26): Prepared according to general procedure A at $100^{\circ} \mathrm{C}$ using 2-(trifluoromethyl)pyridine $(0.5 \mathrm{mmol}, 58 \mu \mathrm{L}, 1.0$ equiv), 3-buten-1-ol (1.5 mmol, $130 \mu \mathrm{L}, 3.0$ equiv), sodium formate $(2.5 \mathrm{mmol}, 0.17 \mathrm{~g}, 5.0$ equiv $)$, mesna ( $0.1 \mathrm{mmol}, 0.016 \mathrm{~g}, 20$ mol\%) and 4CzIPN (0.005 mmol, $0.0039 \mathrm{~g}, 1 \mathrm{~mol} \%)$ in in DMSO (5 mL). The crude product was purified by silica chromatography (20\%-30\% EtOAc/hexanes as the eluent) to afford the title compound as a clear oil ( $0.031 \mathrm{~g}, 30 \%$ yield). The physical and spectral properties were consistent with the reported values. ${ }^{13}$

${ }^{1}$ H NMR (600 MHz, Chloroform-d): $\delta 8.66(\mathrm{~d}, \mathrm{~J} 4.8 \mathrm{~Hz}, 1 \mathrm{H}), 7.81(\mathrm{t}, \mathrm{J}=7.8 \mathrm{~Hz}, 1 \mathrm{H}), 7.64(\mathrm{~d}$, $\mathrm{J}=7.9 \mathrm{~Hz}, 1 \mathrm{H}), 7.36(\mathrm{t}, \mathrm{J}=7.6 \mathrm{~Hz}, 1 \mathrm{H}), 3.66(\mathrm{t}, \mathrm{J}=6.3 \mathrm{~Hz}, 2 \mathrm{H}), 2.37(\mathrm{dp}, \mathrm{J}=16.5 \mathrm{~Hz}, \mathrm{~J}=8.0$ $\mathrm{Hz}, 2 \mathrm{H}), 1.66-1.50(\mathrm{~m}, 4 \mathrm{H})$. 
n-hex $ح$ OH

Octanol (27): Prepared according to general procedure B using octanal $(0.5 \mathrm{mmol}, 78 \mu \mathrm{L}, 1.0$ equiv), sodium formate ( $2.5 \mathrm{mmol}, 0.17 \mathrm{~g}, 5.0$ equiv), mesna $(0.1 \mathrm{mmol}, 0.016 \mathrm{~g}, 20 \mathrm{~mol} \%)$ and 4CzIPN (0.005 mmol, $0.0039 \mathrm{~g}, 1 \mathrm{~mol} \%)$ in 20\% $\mathrm{H}_{2} \mathrm{O} / \mathrm{DMSO}(5 \mathrm{~mL})$. After 16 hours the reaction was purified by silica chromatography (5\%- 30\% EtOAc/hexanes as eluent) to afford the title compound as a yellow oil $(0.057 \mathrm{~g}, 87 \%$ yield $)$. The spectral properties were consistent with the reported values. ${ }^{29}$

${ }^{1}$ H NMR (500 MHz, Chloroform-d) $\delta 3.64(\mathrm{t}, J=6.5 \mathrm{~Hz}, 2 \mathrm{H}), 1.56(\mathrm{p}, J=6.7 \mathrm{~Hz}, 2 \mathrm{H}), 1.39$ $1.22(\mathrm{~m}, 10 \mathrm{H}), 0.88(\mathrm{t}, J=6.6 \mathrm{~Hz}, 3 \mathrm{H})$.<smiles>OCCCc1ccc(Cl)cc1</smiles>

3-(4-chlorophenyl)propanol (28): Prepared according to general procedure B using 3-(4chlorophenyl)propanal $\mathbf{S 1 3}(0.25 \mathrm{mmol}, 0.042 \mathrm{~g}, 1.0$ equiv), sodium formate (1.25 mmol, 0.085 g, 5.0 equiv), mesna (0.05 mmol, $0.0082 \mathrm{~g}, 20 \mathrm{~mol} \%)$ and $4 \mathrm{CzIPN}(0.0025 \mathrm{mmol}, 0.0020 \mathrm{~g}, 1$ mol $\%$ ) in $20 \% \mathrm{H}_{2} \mathrm{O} / \mathrm{DMSO}(2.5 \mathrm{~mL})$. After 16 hours the reaction was purified by silica chromatography (0\%-30\% EtOAc/hexanes as the eluent) to afford the title compound as a pale yellow oil $(0.033 \mathrm{~g}, 80 \%$ yield $)$. The spectral properties were consistent with the reported values. $^{30}$

${ }^{1}$ H NMR (600 MHz, Chloroform-d) $\delta 7.25(\mathrm{~d}, J=8.3 \mathrm{~Hz}, 2 \mathrm{H}), 7.13(\mathrm{~d}, J=8.4 \mathrm{~Hz}, 2 \mathrm{H}), 3.66(\mathrm{t}$, $J=6.4 \mathrm{~Hz}, 2 \mathrm{H}), 2.74-2.65(\mathrm{~m}, 2 \mathrm{H}), 1.92-1.82(\mathrm{~m}, 2 \mathrm{H})$.<smiles>OCC1CCCCC1</smiles>

Cyclohexylmethanol (29): Prepared according to general procedure B using cyclohexanecarbaldehyde $(0.5 \mathrm{mmol}, 60 \mu \mathrm{L}, 1.0$ equiv), sodium formate $(2.5 \mathrm{mmol}, 0.17 \mathrm{~g}, 5.0$ equiv), mesna (0.1 mmol, $0.016 \mathrm{~g}, 20 \mathrm{~mol} \%)$ and $4 \mathrm{CzIPN}(0.005 \mathrm{mmol}, 0.0039 \mathrm{~g}, 1 \mathrm{~mol} \%)$ in $20 \% \mathrm{H}_{2} \mathrm{O} / \mathrm{DMSO}(5 \mathrm{~mL})$. After 16 hours the reaction was purified by silica chromatography ( $0 \%-20 \%$ EtOAc/hexanes as the eluent) to provide the title compound as a pale yellow oil $\left(0.033 \mathrm{~g}, 80 \%\right.$ yield). The spectral properties were consistent with the reported values. ${ }^{31}$

${ }^{1}$ H NMR (600 MHz, Chloroform-d) $\delta 3.44(\mathrm{~d}, J=6.4 \mathrm{~Hz}, 2 \mathrm{H}), 1.86-1.60(\mathrm{~m}, 5 \mathrm{H}), 1.52-1.42$ (m, 1H), $1.31-1.12(\mathrm{~m}, 4 \mathrm{H}), 0.99-0.88(\mathrm{~m}, 2 \mathrm{H})$. 


\section{Carboxylation/ Reduction of Olefins}<smiles>CC(CC(=O)NCCc1ccccc1)C(=O)O</smiles>

2-methyl-4-oxo-4-(phenethylamino)butanoic acid (30): Prepared according to general procedure B using $(E)-\mathrm{N}$-phenethylbut-2-enamide $\mathbf{S 1 5}$ (1 mmol, $0.189 \mathrm{~g}, 1$ equiv), sodium formate ( $5 \mathrm{mmol}, 0.34 \mathrm{~g}, 5.0$ equiv), formic acid $(5 \mathrm{mmol}, 0.19 \mathrm{~mL}, 5$ equiv), mesna $(0.2 \mathrm{mmol}$, $0.032 \mathrm{~g}, 20 \mathrm{~mol} \%$ ) and 4CzIPN (0.01 mmol, $0.0078 \mathrm{~g}, 1 \mathrm{~mol} \%)$ in 20\% $\mathrm{H}_{2} \mathrm{O} / \mathrm{DMSO}(10 \mathrm{~mL})$. After 16 hours under irradiation by Kessil $390 \mathrm{~nm}$ light, the crude product was purified by silica chromatography (10-100\% EtOAc/hexanes $+1 \% \mathrm{AcOH}$ as the eluent) to afford the title compound as a white solid $(0.162 \mathrm{~g}, 69 \%$ yield $)$.

${ }^{1}$ H NMR (400 MHz, Chloroform-d) $\delta 7.31$ (m, 2H), 7.23 (m, 3H), 5.72 (br s, 1H), $3.64-3.47$ $(\mathrm{m}, 2 \mathrm{H}), 3.01-2.87(\mathrm{~m}, 1 \mathrm{H}), 2.83(\mathrm{t}, J=6.9 \mathrm{~Hz}, 2 \mathrm{H}), 2.54(\mathrm{dd}, J=15.6,8.8 \mathrm{~Hz}, 1 \mathrm{H}), 2.30$ (dd, $J=15.5,4.3 \mathrm{~Hz}, 1 \mathrm{H}), 1.23(\mathrm{~d}, J=7.2 \mathrm{~Hz}, 3 \mathrm{H})$.

${ }^{13}$ C NMR (151 MHz, DMSO-d 6 ) $\delta$ 176.72, 170.30, 139.48, 128.61, 128.27, 126.02, 40.15, $38.66,35.45,35.17,16.65$.

HRMS (APCI) $m / z$ : [M+H] calcd. for $\mathrm{C}_{13} \mathrm{H}_{18} \mathrm{NO}_{3}, 236.1281$; found, 236.1285 .

Rf: 0.14 (85\% EtOAc/hexanes)<smiles>CC(CC#N)C(=O)O</smiles>

3-cyano-2-methylpropanoic acid (31): Prepared according to general procedure B using crotonitrile ( $1 \mathrm{mmol}, 0.076 \mathrm{~g}, 1$ equiv), sodium formate ( $5 \mathrm{mmol}, 0.34 \mathrm{~g}, 5.0$ equiv), formic acid ( $5 \mathrm{mmol}, 0.19 \mathrm{~mL}, 5$ equiv), mesna $(0.2 \mathrm{mmol}, 1.032 \mathrm{~g}, 20 \mathrm{~mol} \%)$ and 4CzIPN (0.01 mmol, $0.0078 \mathrm{~g}, 1 \mathrm{~mol} \%)$ in $20 \% \mathrm{H}_{2} \mathrm{O} / \mathrm{DMSO}(10 \mathrm{~mL})$. After 16 hours under irradiation by Kessil 390 $\mathrm{nm}$ light, the crude reaction mixture was diluted with EtOAc $(10 \mathrm{~mL})$ and washed with saturated aqueous potassium carbonate. The organic phase was collected in a flask and the aqueous phase was acidified with concentrated $\mathrm{HCl}$ to $\mathrm{pH}$ 1-2. The aqueous phase was then extracted with EtOAc $(3 \mathrm{x})$ and the combined organic extracts were pushed through a silica plug (100\% EtOAc as the eluent) and concentrated in vacuo to afford the title compound as a yellow oil $(0.071 \mathrm{~g}$, $63 \%$ yield).

${ }^{1}$ H NMR (600 MHz, Chloroform-d): $\delta$ 2.94-2.84 (m, 1H), 2.70 (dd, J = 16.7, 5.91 H, 1H), 2.57 (dd, J = 16.8, 7.4 Hz, 1H), $1.43(\mathrm{~d}, \mathrm{~J}=7.2 \mathrm{~Hz}, 3 \mathrm{H})$.

${ }^{13}$ C NMR (151 MHz, Chloroform-d) $\delta$ 178.31, 117.59, 77.37, 77.16, 76.95, 36.09, 20.93, 16.60.

HRMS (APCI) $m / z$ : $[\mathrm{M}+\mathrm{H}]$ calcd. for $\mathrm{C}_{5} \mathrm{H}_{8} \mathrm{NO}_{2}, 114.0550$; found, 114.0553.

Rf: 0.35 (50\% EtOAc/hexanes) 
<smiles>CCOC(=O)CC(C(=O)O)C1CC1</smiles>

2-cyclopropyl-4-ethoxy-4-oxobutanoic acid (32): Prepared according to general procedure B using ethyl (E)-3-cyclopropylacrylate $\mathbf{S 1 6}$ (1 mmol, $0.140 \mathrm{~g}, 1$ equiv), sodium formate (5 mmol, $0.34 \mathrm{~g}, 5.0$ equiv), formic acid ( $5 \mathrm{mmol}, 0.19 \mathrm{~mL}, 5$ equiv), mesna ( $0.2 \mathrm{mmol}, 0.032 \mathrm{~g}, 20 \mathrm{~mol} \%)$ and 4CzIPN (0.01 mmol, $0.0078 \mathrm{~g}, 1 \mathrm{~mol} \%)$ in $\mathrm{H}_{2} \mathrm{O} / \mathrm{DMSO}(10 \mathrm{~mL})$. After 16 hours under irradiation by Kessil $390 \mathrm{~nm}$ light, the crude product was purified by silica chromatography (10$70 \%$ EtOAc/hexanes $+1 \% \mathrm{AcOH}$ as the eluent) to afford the title compound as a yellow oil (0.122 g, 66\% yield).

${ }^{1}$ H NMR (600 MHz, Chloroform-d) $\delta 4.24-3.95(\mathrm{~m}, 2 \mathrm{H}), 2.82(\mathrm{dd}, J=16.1,10.2 \mathrm{~Hz}, 1 \mathrm{H})$, $2.59(\mathrm{dd}, J=16.1,5.5 \mathrm{~Hz}, 1 \mathrm{H}), 2.06(\mathrm{td}, J=9.7,4.9 \mathrm{~Hz}, 1 \mathrm{H}), 1.24(\mathrm{t}, J=7.1 \mathrm{~Hz}, 3 \mathrm{H}), 0.97-$ $0.80(\mathrm{~m}, 1 \mathrm{H}), 0.63-0.52(\mathrm{~m}, 2 \mathrm{H}), 0.52-0.43(\mathrm{~m}, 1 \mathrm{H}), 0.25-0.16(\mathrm{~m}, 1 \mathrm{H})$.

${ }^{13}$ C NMR (151 MHz, Chloroform-d) $\delta 180.30,171.96,60.88,46.27,36.53,14.23,13.59,4.44$. HRMS (APCI) $m / z$ : [M+H] calcd. for $\mathrm{C}_{9} \mathrm{H}_{15} \mathrm{O}_{4}, 187.0965$; found, 187.0968 .

Rf: $0.52(50 \%$ EtOAc/hexanes)<smiles>CCC=CCC(=O)CCCc1ccccc1</smiles>

(E)-1-phenylnon-6-en-4-one (33): Prepared according to general procedure B using (E)-1cyclopropyl-6-phenylhex-1-en-3-one $\mathbf{S 1 7}$ ( $1 \mathrm{mmol}, 0.214 \mathrm{~g}, 1$ equiv), sodium formate (5 mmol, $0.34 \mathrm{~g}, 5.0$ equiv), formic acid ( $5 \mathrm{mmol}, 0.19 \mathrm{~mL}, 5$ equiv), mesna $(0.2 \mathrm{mmol}, 0.032 \mathrm{~g}, 20 \mathrm{~mol} \%)$ and $4 \mathrm{CzIPN}$ (0.01 mmol, $0.0078 \mathrm{~g}, 1 \mathrm{~mol} \%)$ in 20\% $\mathrm{H}_{2} \mathrm{O} / \mathrm{DMSO}(10 \mathrm{~mL})$. After 16 hours under irradiation by Kessil $390 \mathrm{~nm}$ light, the crude product was purified by silica chromatography (5$20 \% \mathrm{EtOAc} /$ hexanes as the eluent) to afford the title compound as a mixture of E/Z isomers as a clear oil ( $0.168 \mathrm{~g}, 77 \%$ yield).

${ }^{1}$ H NMR (600 MHz, Chloroform-d): $\delta 7.28(\mathrm{t}, \mathrm{J}=7.4 \mathrm{~Hz}, 2 \mathrm{H}), 7.21-7.15(\mathrm{~m}, 3 \mathrm{H}), 5.61-5.53$ $(\mathrm{m}, 1 \mathrm{H}), 5.51-5.46(\mathrm{~m}, 1 \mathrm{H}), 3.14-3.12(\mathrm{~d}, \mathrm{~J}=7.1 \mathrm{~Hz}, 0.37 \mathrm{H}, \mathrm{Z}$ isomer $), 3.07(\mathrm{~d}, \mathrm{~J}=6.7 \mathrm{~Hz}, 1.70$ $\mathrm{H}, \mathrm{E}$ isomer), $2.61(\mathrm{t}, \mathrm{J}=7.6 \mathrm{~Hz}, 2 \mathrm{H}), 2.44(\mathrm{t}, \mathrm{J}=7.1 \mathrm{~Hz}, 2 \mathrm{H}), 2.08-1.99(\mathrm{~m}, 2 \mathrm{H}), 1.94-1.87(\mathrm{~m}$, $2 \mathrm{H}), 0.98(\mathrm{t}, \mathrm{J}=7.3 \mathrm{~Hz}, 3 \mathrm{H})$.

${ }^{13}$ C NMR (151 MHz, Chloroform-d) $\delta$ 209.50, 208.89, 141.78, 136.84, 135.46, 128.61, 128.51, 126.07, 121.01, 120.38, 46.97, 41.78, 41.53, 41.38, 35.20, 25.75, 25.33, 25.26, 20.95, 14.06, 13.66.

HRMS (APCI) $m / z$ : [M+H] calcd. for $\mathrm{C}_{15} \mathrm{H}_{21} \mathrm{O}, 217.1587$; found, 217.1590.

Rf: $0.60(10 \%$ EtOAc/hexanes $)$<smiles>COC(=O)CCc1ccccn1</smiles>

Methyl 3-(pyridin-2-yl)propanoate (34): Prepared according to general procedure B using methyl (E)-3-(pyridine-2-yl)acrylate $\mathbf{S 1 8}$ (1 mmol, $0.163 \mathrm{~g}, 1$ equiv), sodium formate (5 mmol, 
$0.34 \mathrm{~g}, 5.0$ equiv), formic acid ( $5 \mathrm{mmol}, 0.19 \mathrm{~mL}, 5$ equiv), mesna ( $0.2 \mathrm{mmol}, 0.032 \mathrm{~g}, 20 \mathrm{~mol} \%)$ and 4CzIPN (0.01 mmol, $0.0078 \mathrm{~g}, 1 \mathrm{~mol} \%)$ in $0.1 \mathrm{M} 20 \% \mathrm{H}_{2} \mathrm{O} / \mathrm{DMSO}(10 \mathrm{~mL})$. After 16 hours under irradiation by Kessil $390 \mathrm{~nm}$ light, the crude product was by silica chromatography (30$50 \% \mathrm{EtOAc} / \mathrm{h}$ exanes as the eluent) to afford the title compound as a colorless oil $(0.157 \mathrm{~g}, 95 \%$ yield). The physical and spectral properties are consistent with the reported values. ${ }^{32}$

${ }^{1} \mathbf{H}$ NMR (600 MHz, CDCl $\left.\mathbf{~}_{3}\right): \delta 8.51(\mathrm{~d}, \mathrm{~J}=3.2 \mathrm{~Hz}, 1 \mathrm{H}), 7.59(\mathrm{td}, \mathrm{J}=7.7,2.0 \mathrm{~Hz}, 1 \mathrm{H}), 7.18(\mathrm{~d}, \mathrm{~J}$ $=7.8 \mathrm{~Hz}, 1 \mathrm{H}), 7.14-7.07(\mathrm{~m}, 1 \mathrm{H}), 3.66(\mathrm{~s}, 3 \mathrm{H}), 3.11(\mathrm{t}, \mathrm{J}=7.5 \mathrm{~Hz}, 2 \mathrm{H}), 2.81(\mathrm{t}, \mathrm{J}=7.5 \mathrm{~Hz}, 2 \mathrm{H})$. 


\section{Alternative Initiators}

\section{Alternative Initiators Procedure}

An $8 \mathrm{~mL}$ screw-top test tube was charged with appropriate initiator ( $20 \mathrm{~mol} \%, 0.02 \mathrm{~mol}$ ), sodium formate (5.0 equiv, $0.5 \mathrm{mmol})$ and mesna $(20 \mathrm{~mol} \%, 0.02 \mathrm{~mol}$, if needed-refer to table below). The tube was equipped with a stir bar and sealed with a PTFE/silicon septum. The atmosphere was exchanged by applying vacuum and backfilling with nitrogen (this process was conducted a total of three times). Under nitrogen atmosphere, separately degassed DMSO $(0.1 \mathrm{M}, 1 \mathrm{~mL})$ was added via syringe, followed by 2 -chloromethylbenzoate ( 1 equiv, $0.1 \mathrm{mmol}$ ). The resulting mixture was stirred at $1400 \mathrm{RPM}$ for $16 \mathrm{~h}$ under irradiation by blue LEDs or heated to $100{ }^{\circ} \mathrm{C}$ (refer to table below). The reaction was then extracted with ethyl acetate $(3 \mathrm{x})$. The organic layer was passed through a small silica plug with $100 \%$ EtOAc and concentrated. Deutro-chloroform with an internal standard of dibromomethane $(7 \mu \mathrm{L}, 0.1 \mathrm{mmol})$ was added. The sample was analyzed by ${ }^{1} \mathrm{H}$ NMR $(\mathrm{d}=5 \mathrm{~s})$, and the integral values were used to calculate the data given in Table S4.

Initiators:

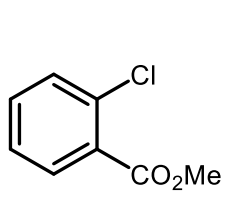

SM initiator

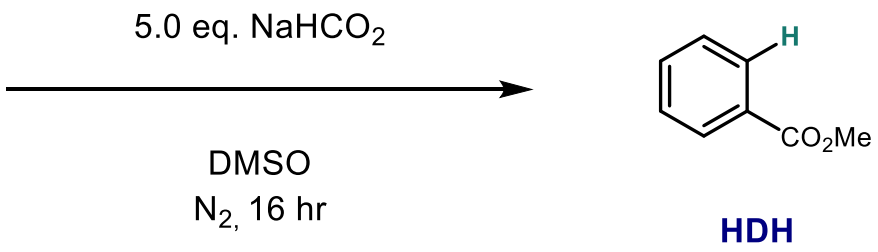

\begin{tabular}{cccc}
\hline Entry & initiator & SM & HDH \\
\hline 1 & $20 \mathrm{~mol} \%$ methyl disulfide, blue LEDs & 0 & 99 \\
2 & $20 \mathrm{~mol} \%$ ammonium persulfate, 20 mol\% mesna, $100{ }^{\circ} \mathrm{C}$ & 0 & 96 \\
3 & $20 \mathrm{~mol} \% \mathrm{AlBN}, 20 \mathrm{~mol} \%$ mesna, $100{ }^{\circ} \mathrm{C}$ & 50 & 48 \\
\hline
\end{tabular}

yields determined by ${ }^{1} \mathrm{H}$ NMR with dibromomethane as internal standard

Table S4. 


\section{Fluorescence Quenching Experiments}

Fluorescence spectra is measured on a Horiba Fluoromax Plus C. The sample is placed in a $1 \mathrm{~cm}$ pathlength cell. Quenching studies were conducted in DMSO with the photocatalyst (4CzIPN) concentration of $70 \mu \mathrm{M}$ with varying concentrations of quenchers. All samples were prepared and stored under a nitrogen atmosphere. The sample is placed in a $1 \mathrm{~cm}$ pathlength cell. The sample is excited at $435 \mathrm{~nm}$ and the PL spectra is detected with $1 \mathrm{~nm}$ resolution at a 90 degree angle. Data points were fitted with a linear trend.

(A)

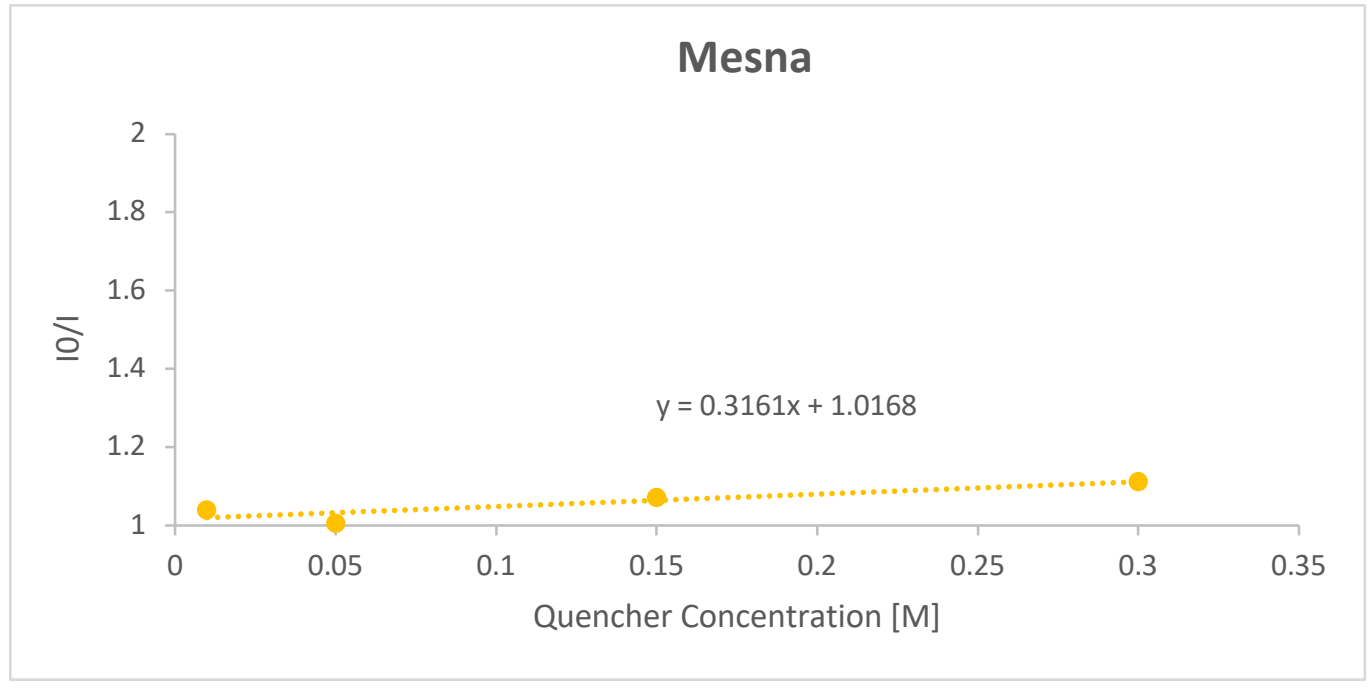

(B)

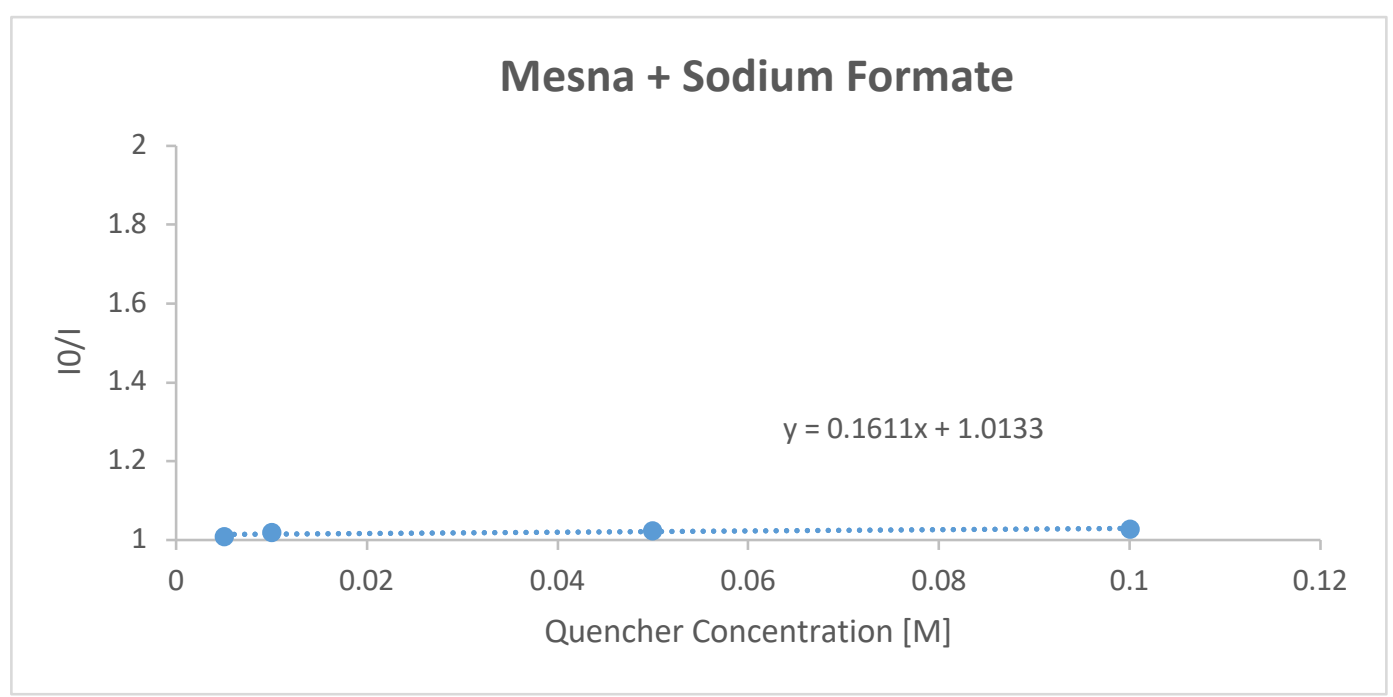

Figure S1. (a) Stern- Volmer plot with Mesna (b) Stern-Volmer plot with Mesna and sodium formate to assess if PCET is occuring ${ }^{33}$ 


\section{Transient absorption spectroscopy}

\section{Methods}

Transient spectroscopy is performed on Ultrafast Systems Helios and EOS system. The laser system is based on a Coherent Legend regenerative amplifier system, with 150 fs pulse width, 1 $\mathrm{kHz}$ repetition rate, and $2 \mathrm{~mJ}$ pulse energy. For the measurement within $1 \mathrm{~ns}$, the fundamental $800 \mathrm{~nm}$ light is split to generate the $400 \mathrm{~nm}$ excitation light via a BBO crystal (pump) and a broadband whitelight extended into UV region by focusing on a rotating $\mathrm{CaF}_{2}$ window (probe). The pump and probe are focused on the sample cell $(1 \mathrm{~mm})$ and the probe light is collected via a fiber. For longer delay time the whitelight is generated by a supercontinuum laser. The detection scheme is similar. The data is analyzed by Surface Xplorer and Igor Pro.

\section{Analysis}

Transient spectroscopy is employed to investigate the single electron transfer (SET) from the photocatalyst (4CzIPN) to the thiol (mesna) at the initialization of the reaction. First, we studied the photocatalyst by exciting at $400 \mathrm{~nm}$, at the ground state absorption. The transient absorption spectrum shows an intersystem crossing process of $4 \mathrm{CzIPN}$ from singlet to triplet excited state at delay time ranging from $10 \mathrm{~ns}$ to $10 \mu \mathrm{s}$ as shown in Figure S3a, S3b, and S3c. The singlet and triplet excited state shows similar ground state bleach (GSB, 390-400 nm, Figure S2a and S2b) and broad excited state absorption (ESA, $\sim 450-900 \mathrm{~nm}$ ) position, but different amplitude. The SET is observed by comparing the kinetics at one of the ESA signal at $473 \mathrm{~nm}$, where the triplet state of 4CzIPN decays faster with more thiol added as shown in Figure S3c. The SET occurs on a relatively slow time scale where the singlet state decay away as both systems show no difference within $1 \mathrm{~ns}$ as shown in Figure S2. Since the excited 4CzIPN concentration is significant lower (more than 1000 times) than the thiol concentration, the bimolecular reaction rate can be simplified to a pseudo-first order reaction.

\section{Fitting functions and quantum yield calculation}

The singlet and triplet lifetime of the photocatalyst can be fitted with a bi-exponential function to the kinetics shown in Figure 1c:

$$
\Delta A=\Delta A_{0}\left(a_{1} e^{k_{1} t}+a_{2} e^{k_{2} t}\right)
$$

where $k_{1}$ and $k_{2}$ are the decay rate of the singlet and triplet state. From the fitting shown in Figure $\mathrm{S} 3 \mathrm{~d}, k_{l}=13.8 \pm 0.1 \mathrm{~ns}^{-1}, k_{2}=1.80 \pm 0.06 \mu \mathrm{s}^{-1}$.

With the thiol in the system, since the SET occurs in the triplet state, the additional decay rate $k$ leads to faster reaction, which is:

$$
\Delta A=\Delta A_{0}\left(a_{1} e^{k_{1} t}+a_{2} e^{\left(k_{2}+k\right) t}\right)
$$

Fitting result at different concentrations in Figure S3d gives the $k$ value at different concentration and the pseudo-first order reaction rate $k_{0}$ can be obtained by:

$$
k_{0}=k[\text { thiol }]=0.402 \mu s^{-1} M^{-1}
$$

Although the absolute quantum yield of triplet state of the photocatalyst cannot be determined by the transient study, we can estimate the upper limit of the quantum yield of the SET from triplet state by assuming a unity intersystem crossing efficiency. 
The upper limit of SET quantum yield in real experimental condition $(0.01 \mathrm{M}$ thiol) can be calculated by:

$$
Q Y_{S E T}=\frac{k_{E T}[\text { thiol }]}{k_{E T}[\text { thiol }]+k_{\text {triplet }}}=\frac{0.402 * 0.01}{0.402 * 0.01+1 / 1.80}=0.72 \%
$$

(a)
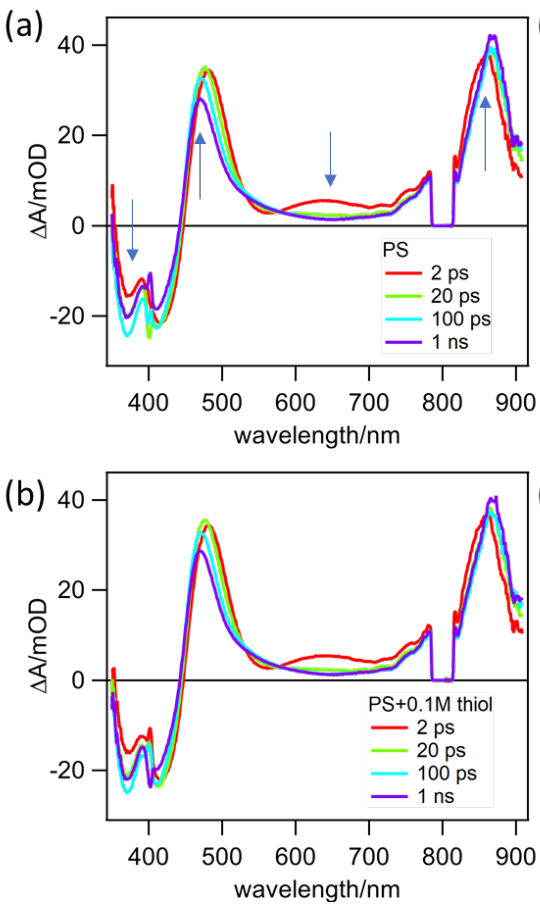
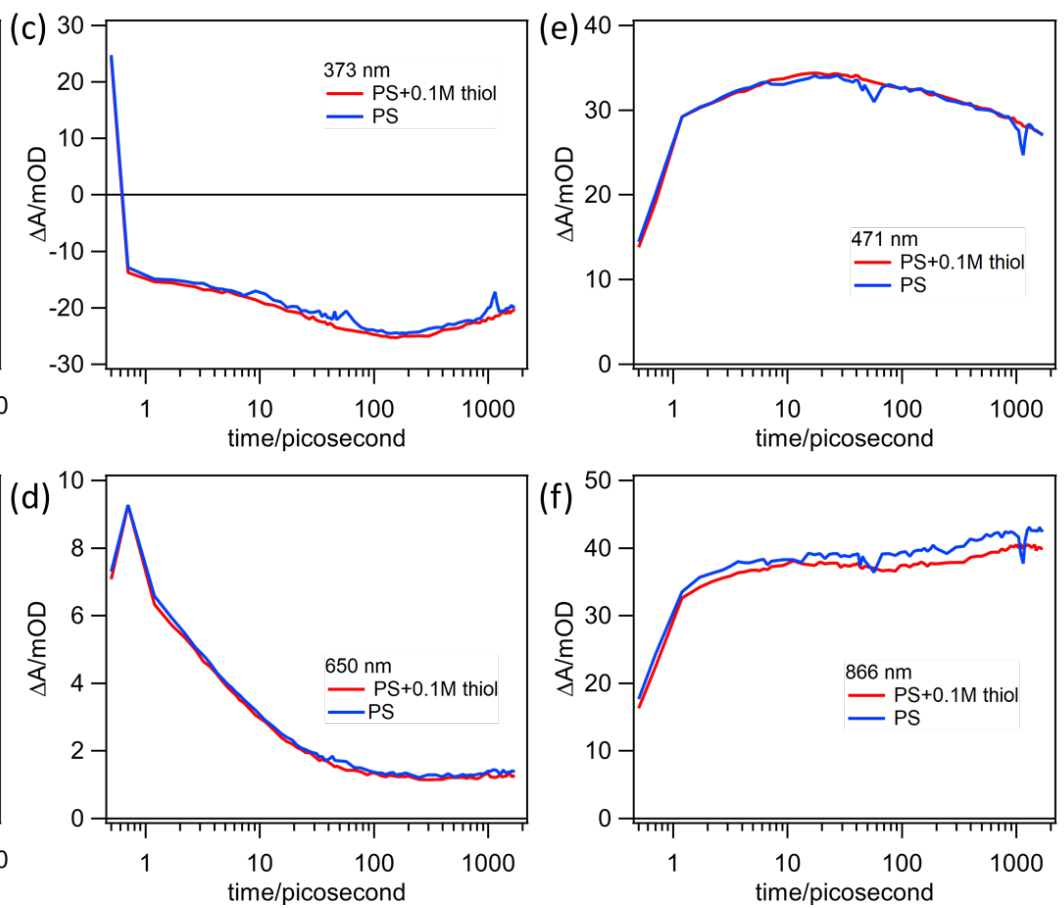

Figure S2. TAS of photocatalyst (4CzIPN) and with $0.1 \mathrm{M}$ thiol within $1 \mathrm{~ns}$ time window. (a)-(b) TA spectra of PS and PS+0.1M thiol. (c)-(f) Kinetics comparison at various indicated wavelength. The spectra and kinetics show no difference at this time range. 

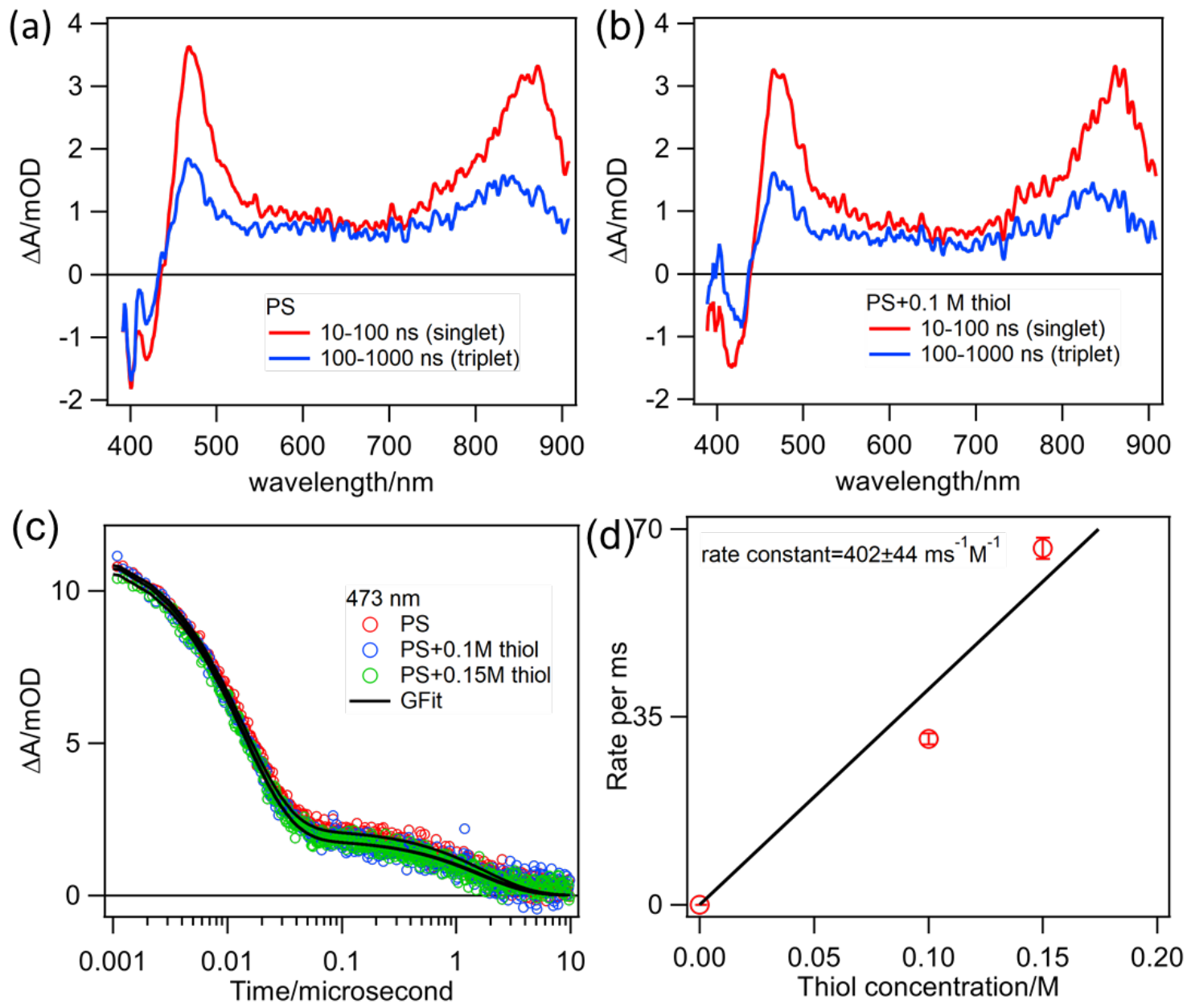

Figure S3. Transient absorption spectroscopy (TAS) of the photocatalyst (4CzIPN) with thiol (mesna). (a) TA spectra of 4CzIPN and (b) 4CzIPN with $0.1 \mathrm{M}$ thiol after $400 \mathrm{~nm}$ excitation with delay time at 10-100 ns and 100-1000 ns, which corresponds to the singlet and triplet excited state, respectively. (c) Kinetics probed at $473 \mathrm{~ns}$ of $4 \mathrm{CzIPN}, 4 \mathrm{CzIPN}$ with $0.1 \mathrm{M}$ and $0.15 \mathrm{M}$ thiol. The higher concentration of thiol leads to faster decay of triplet state. (d) The thiol induced quenching rate of the triplet state is plotted against the thiol concentration, the fitting gives the pseudo-first order rate constant of thiol quenching to be $402 \mathrm{~ms}^{-1} \mathrm{M}^{-1}$. 

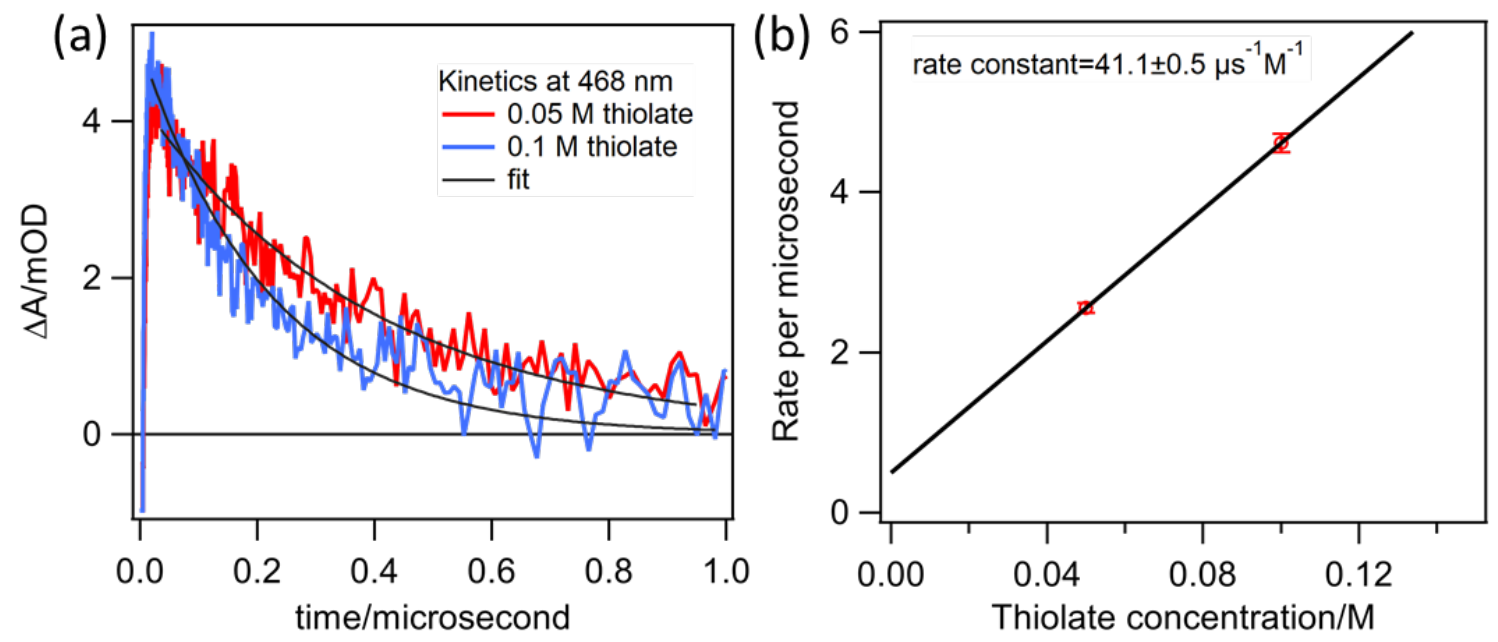

Figure S4. (a) Quenching kinetics of thiolate (sodium thiomethoxide) with photocatalyst (4CzIPN) at $0.05 \mathrm{M}$ and $0.1 \mathrm{M}$ concentration. (b) Pseudo-first order rate constant of thiolate. 


\section{Quantum Yield Experiment}

Quantum Yield Experiments were performed according to the procedure of Yoon. ${ }^{34}$

\section{Determination of the light intensity at $436 \mathrm{~nm}$ :}

The photon flux of the spectrophotometer was determined by standard ferrioxalate actinometry. A $0.15 \mathrm{M}$ solution of ferrioxalate was prepared by dissolving $2.21 \mathrm{~g}$ of potassium ferrioxalate hydrate in $30 \mathrm{~mL}$ of $0.05 \mathrm{M} \mathrm{H}_{2} \mathrm{SO}_{4}$. A buffered solution of phenanthroline was prepared by dissolving $50 \mathrm{mg}$ of phenanthroline and $11.25 \mathrm{~g}$ of sodium acetate in $50 \mathrm{~mL}$ of 0.5 $\mathrm{M} \mathrm{H}_{2} \mathrm{SO} 4$. Both solutions were covered in foil and stored in the dark. To determine the photon flux of our lamp source, $2.0 \mathrm{~mL}$ of the ferrioxalate solution was placed in an $8 \mathrm{~mL}$ screw-top test tube and irradiated for 10.0s with a blue LED lamp ( $\lambda=435 \mathrm{~nm}$, LED wholesalers PAR38 Indoor Outdoor 16-Watt LED Flood Light Bulb, Blue) at a distance of exactly $7.0 \mathrm{~cm}$. After irradiation, $0.35 \mathrm{~mL}$ of the phenanthroline solution was added to the test tube. The solution was then allowed to rest for $1 \mathrm{~h}$ in the dark to allow the ferrous ions to completely coordinate to the phenanthroline. The absorbance of the solution was measured at $510 \mathrm{~nm}$. Conversion was calculated using eq. 1 .

$$
\text { (1) } \mathrm{mol} \mathrm{Fe}^{2+}=\frac{V \cdot \Delta A}{\mathrm{l} \cdot \varepsilon}
$$

Where $\mathrm{V}$ is the total volume $(0.00235 \mathrm{~L})$ of the solution after addition of phenanthroline, $\Delta \mathrm{A}$ is the difference in absorbance at $510 \mathrm{~nm}$ between the irradiated and non-irradiated solutions, 1 is the path length $(1.000 \mathrm{~cm})$, and $\varepsilon$ is the molar absorptivity at $510 \mathrm{~nm}\left(11,100 \mathrm{~L} \mathrm{~mol}^{-1} \mathrm{~cm}^{-1}\right){ }^{18}$ The photon flux can be calculated using eq 2 .

$$
\text { (2) Photon flux }=\frac{\mathrm{mol} \mathrm{Fe} 2+}{\Phi \cdot t \cdot f}
$$

Where $\Phi$ is the quantum yield for the ferrioxalate actinometer (1.01 for a $0.15 \mathrm{M}$ solution at $\lambda=436 \mathrm{~nm}),{ }^{18} \mathrm{t}$ is the time $(10.0 \mathrm{~s})$, and $\mathrm{f}$ is the fraction of light absorbed at $\lambda=436 \mathrm{~nm}$ $(0.99833 \text {, vide infra })^{18}$. The photon flux was calculated (average of three experiments) to be 4.02 $\times 10^{-8}$ einstein $\mathrm{s}^{-1}$.

Sample Calculation:

$$
\begin{aligned}
& \text { Mol Fe } \\
& 2+=\frac{0.0235 \mathrm{~L} \cdot 1.91328}{1.00 \mathrm{~cm} \cdot 11,100 \mathrm{~L} \mathrm{~mol}^{-1} \mathrm{~cm}^{-1}}=4.05 \times 10^{-7} \mathrm{~mol} \\
& \text { Photon flux }=\frac{4.05 \times 10^{-7} \mathrm{~mol}}{1.01 \cdot 10 \mathrm{~s} \cdot 0.99833}=4.02 \times 10^{-8} \text { einsteins } \mathrm{s}^{-}
\end{aligned}
$$




\section{Determination of quantum yield}
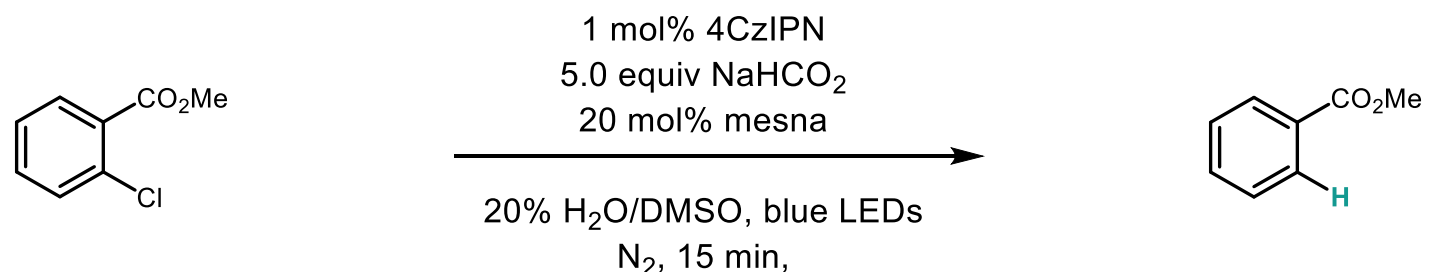

$\mathrm{N}_{2}, 15 \mathrm{~min}$,

An $8 \mathrm{~mL}$ screw-top test tube was charged with $4 \mathrm{CzIPN}(0.002,29 \mu \mathrm{L}, 1 \mathrm{~mol} \%)$, sodium formate ( $1.0 \mathrm{mmol}, 0.068 \mathrm{~g}, 5.0$ equiv) and mesna $(0.04,20 \mathrm{~mol} \%, 0.02 \mathrm{~mol})$. The tube was equipped with a stir bar and sealed with a PTFE/silicon septum. Under nitrogen atmosphere, separately degassed DMSO (2 mL) was added via syringe, followed by 2-chloromethylbenzoate $(0.2 \mathrm{mmol}$, $29 \mu \mathrm{L}, 1.0$ equiv). The resulting mixture was stirred at $1400 \mathrm{RPM}$ for $15 \mathrm{~min}$. under irradiation by blue LEDs at a distance of exactly $7.0 \mathrm{~cm}$. The reaction was then extracted with ethyl acetate $(3 \mathrm{x})$. The organic layer was passed through a small silica plug with $100 \%$ EtOAc and concentrated. Deutro-chloroform with an internal standard of dibromomethane $(14 \mu \mathrm{L}, 0.2$ mmol) was added. The sample was analyzed by ${ }^{1} \mathrm{H}$ NMR $(\mathrm{d}=5 \mathrm{~s})$, and the integral values were used to calculate the data. Essentially all incident light ( $\mathrm{f}>0.999$, vide infra) is absorbed by the $4 \mathrm{CzIPN}$ at the reaction conditions described above. The quantum yield was calculated using eq. 3 the average yield of three experiments to be 2.63 .

$$
\text { (3) } \Phi=\frac{\text { mol product }}{\text { flux } \bullet \text { t } \bullet \mathrm{f}}
$$

Average yield of 3 experiments: $47 \%$ yield (avg. $\mathrm{mmol}=0.095$ )

Sample quantum yield calculation:

$$
\Phi=\frac{9.5 \times 10^{-5} \mathrm{~mol}}{4.02 \times 10^{-8} \cdot 900 \mathrm{~s} \cdot 1.00}=2.63
$$




\section{Chain Length Approximation}

\section{Chain length}

Chain length values calculated in this paper are a lower limit approximation of the actual chain lengths and were calculated using eq. 4, where QY (initial SET) was calculated through transient absorption spectroscopy experimentation (refer to pg. $\mathbf{3 0}$ for calculation).

$$
\text { (4) Chain length }=\frac{\Phi(\text { overall reation })}{\Phi(\text { inital SET })}
$$

Sample Calculation:

$$
\text { Chain length }=\frac{2.63}{0.0072}=365
$$

The lower limit of the chain length was determined to be 365 .

\section{Computational Details}

\section{General.}

All DFT calculations were carried out using the Gaussian 9 software package ${ }^{35}$ at the (U)B3LYP ${ }^{36}$ or R(B3LYP) ${ }^{36}$ level of theory with the $6-311+\mathrm{G}(\mathrm{d}, \mathrm{p})^{37}$ basis set. The CPCM formalism for the Self Consistent Reaction Field (SCRF) model of solvation was employed in calculations to account for solvation in DMSO, and the default parameters as implemented in Gaussian were used.

\section{XII-A Calculated Bond Dissociation Energies}

Calculations were carried out at the (U)B3LYP ${ }^{35}$ level of theory with the $6-311+\mathrm{G}(\mathrm{d}, \mathrm{p})^{37}$ basis set Bond dissociation energies (BDEs) were calculated according to the following equation:

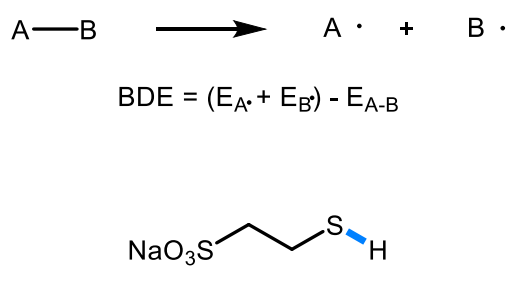<smiles>O=CO[Na]</smiles> 


\section{XII-B. Calculated Reduction Potentials}

Reduction potentials were calculated using a modified procedure as described by Nicewicz and coworkers. ${ }^{38}$ Geometry optimizations were carried out for the reduced and neutral forms of each molecule, and frequency calculations were performed on the minimized structures to ensure no imaginary frequencies existed. Geometry optimizations that did not converge to an energy minimum upon the initial calculation were sequentially optimized using a tight convergence criteria. Gibbs free energies $\left(\mathrm{G}_{298}\right)$ were obtained from the calculation and employed in the following equation:

$$
E_{1 / 2}^{0, \text { calc }}=-\frac{\left(G_{298}[\text { reduced }]-G_{298}[\text { oxidized }]\right)}{n_{e} \mathcal{F}}-E_{1 / 2}^{0, S H E}+E_{1 / 2}^{0, S C E}
$$

Where $n_{e}$ is the number of electrons transferred $\left(n_{e}=1\right.$ for all calculations here), $\mathcal{F}$ is the Faraday constant (value $23.061 \mathrm{kcal} \mathrm{mol}^{-1} \mathrm{~V}^{-1}$ ), $E_{1 / 2}^{0, S H E}$ is the absolute value for the standard hydrogen electrode (SHE, value $=4.281 \mathrm{~V}$ ) and $E_{1 / 2}^{0, S C E}$ is the potential of the saturated calomel electrode (SCE) relative to the SHE in DMSO (value $=-0.279$ ), ${ }^{39}$ and $\mathrm{G}_{298}$ [oxidized] and $\mathrm{G}_{298}$ [reduced] are the Gibbs free energies in DMSO obtained from DFT calculations.<smiles>CC=CC(=O)NN(C)C</smiles>

$-2.67 \mathrm{~V}$

$\mathrm{E}_{1 / 2}^{\text {red }}$ vs SCE $=$<smiles>CC=CC(C)=O</smiles>

$-2.11 \mathrm{~V}$<smiles>C/C=C/C#N</smiles>

$-2.44 \mathrm{~V}$<smiles>COC(=O)/C=C/c1cccnc1</smiles>

$-1.59 \mathrm{~V}$<smiles>CC=CC(=O)OC</smiles>

$-2.35 \mathrm{~V}$

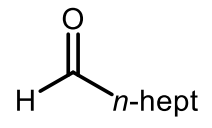

$-2.15 \mathrm{~V}$ 


\section{Electrochemical Measurements}

Electrochemical potentials were obtained with a standard set of conditions according to literature procedure.$^{38}$ Cyclic voltammograms (CVs) were collected with a VersaSTAT 4Potentiostat. Samples were prepared with $0.1 \mathrm{mmol}$ of substrate in $10 \mathrm{~mL}$ of $0.1 \mathrm{M}$ tetra-nbutylammonium hexafluorophosphate in dry, degassed DMSO. Measurements employed a glassy carbon working electrode, platinum wire counter electrode, $3 \mathrm{M} \mathrm{NaCl}$ silver-silver chloride reference electrode, and a scan rate of $100 \mathrm{mV} / \mathrm{s}$. Reductions were measured by scanning potentials in the negative direction and oxidations in the positive direction; the glassy carbon electrode was polished between each scan. Data was analyzed using Microsoft Excel by subtracting a background current prior to identifying the maximum current $(\mathrm{Cp})$ and determining the potential $(\mathrm{Ep} / 2)$ at half this value $(\mathrm{Cp} / 2)$. The obtained value was referenced to $\mathrm{Ag} \mid \mathrm{AgCl}$ and converted to SCE by subtracting $0.035 \mathrm{~V}$.

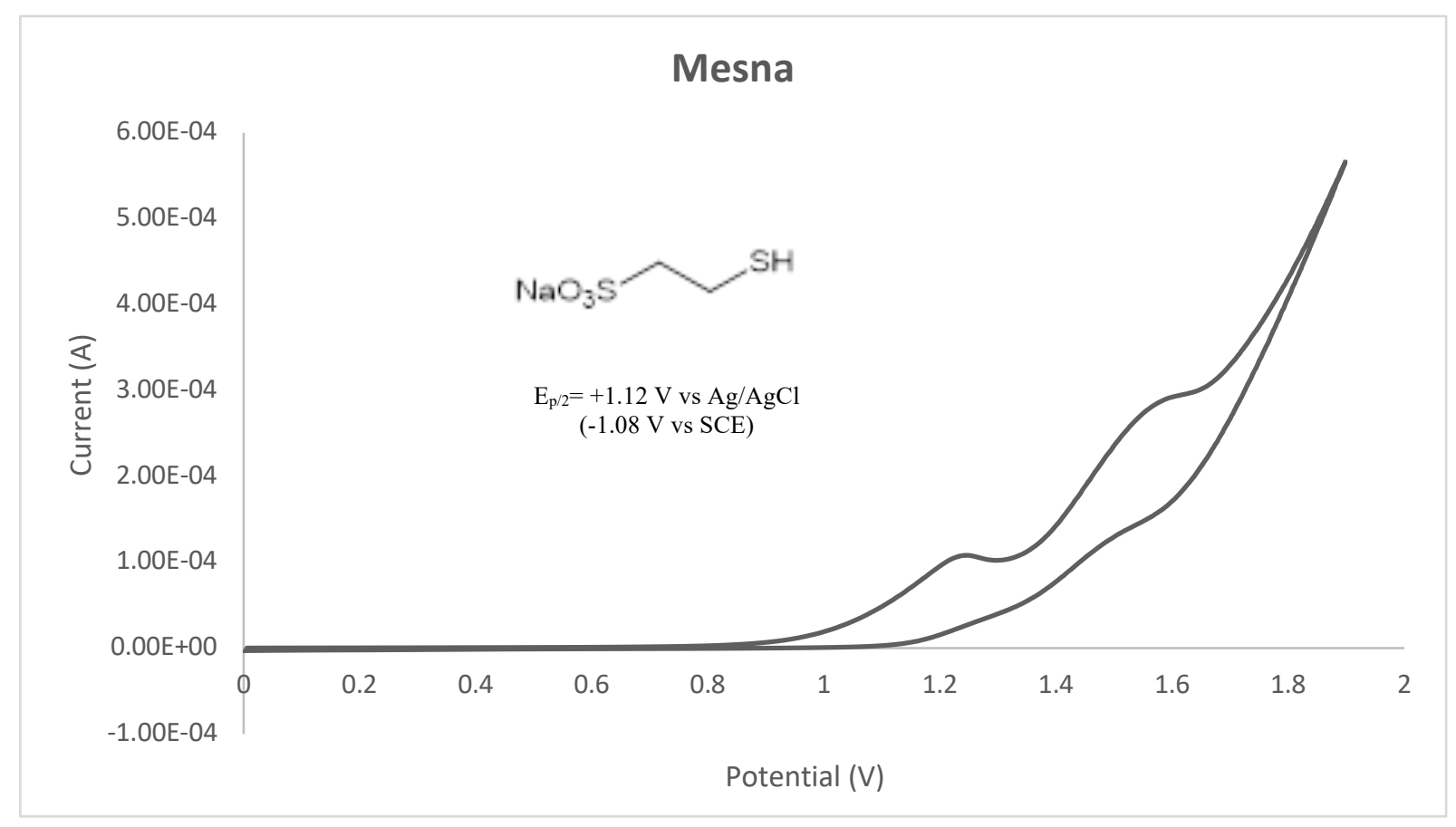

Figure S5. CV of Mesna 


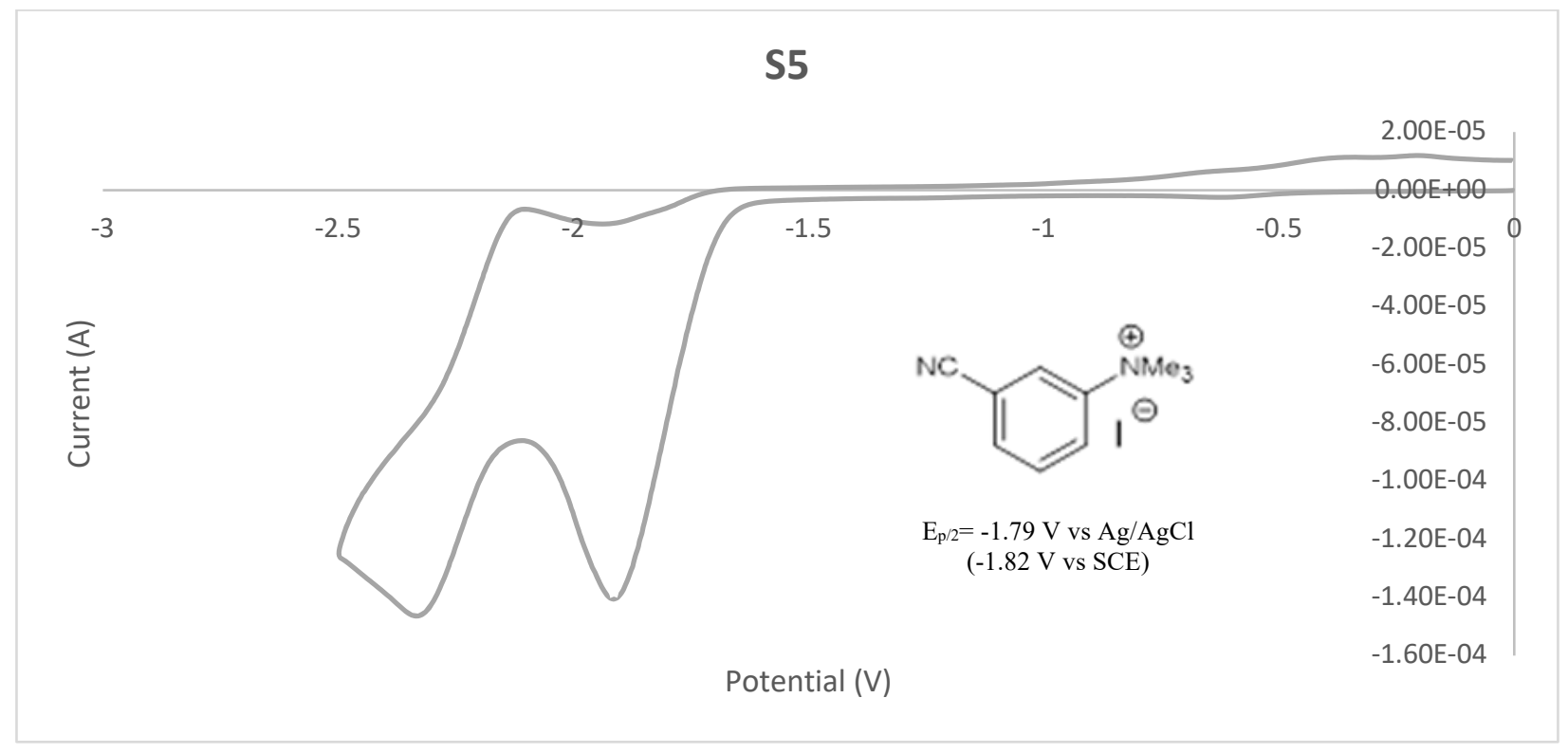

Figure S6. CV of 3-cyano-N,N,N-trimethylbenzenaminium iodide (S5)

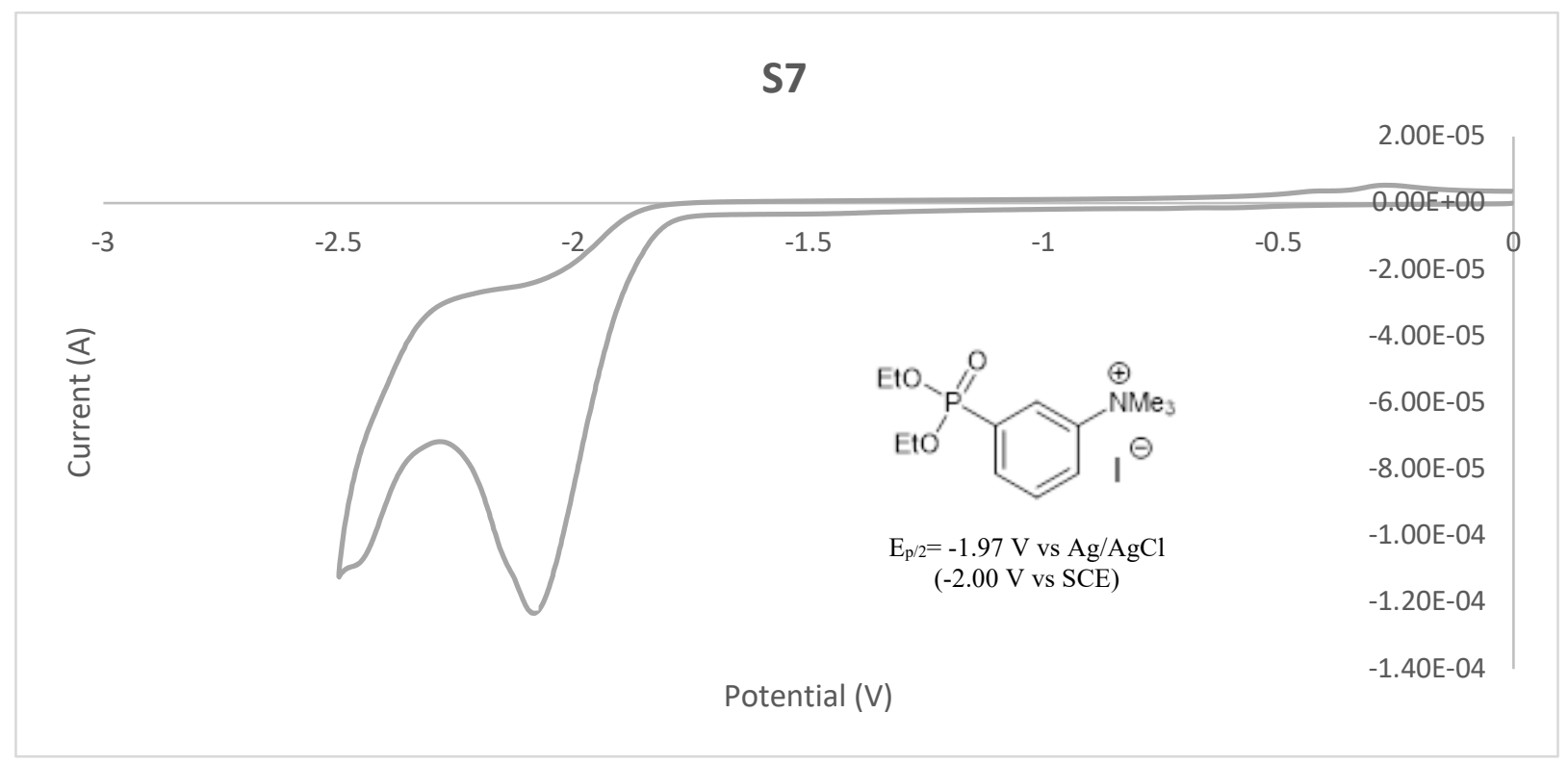

Figure S7. CV of 3-(diethoxyphosphoryl)-N,N,N-trimethylbenzenaminium iodide (S7) 


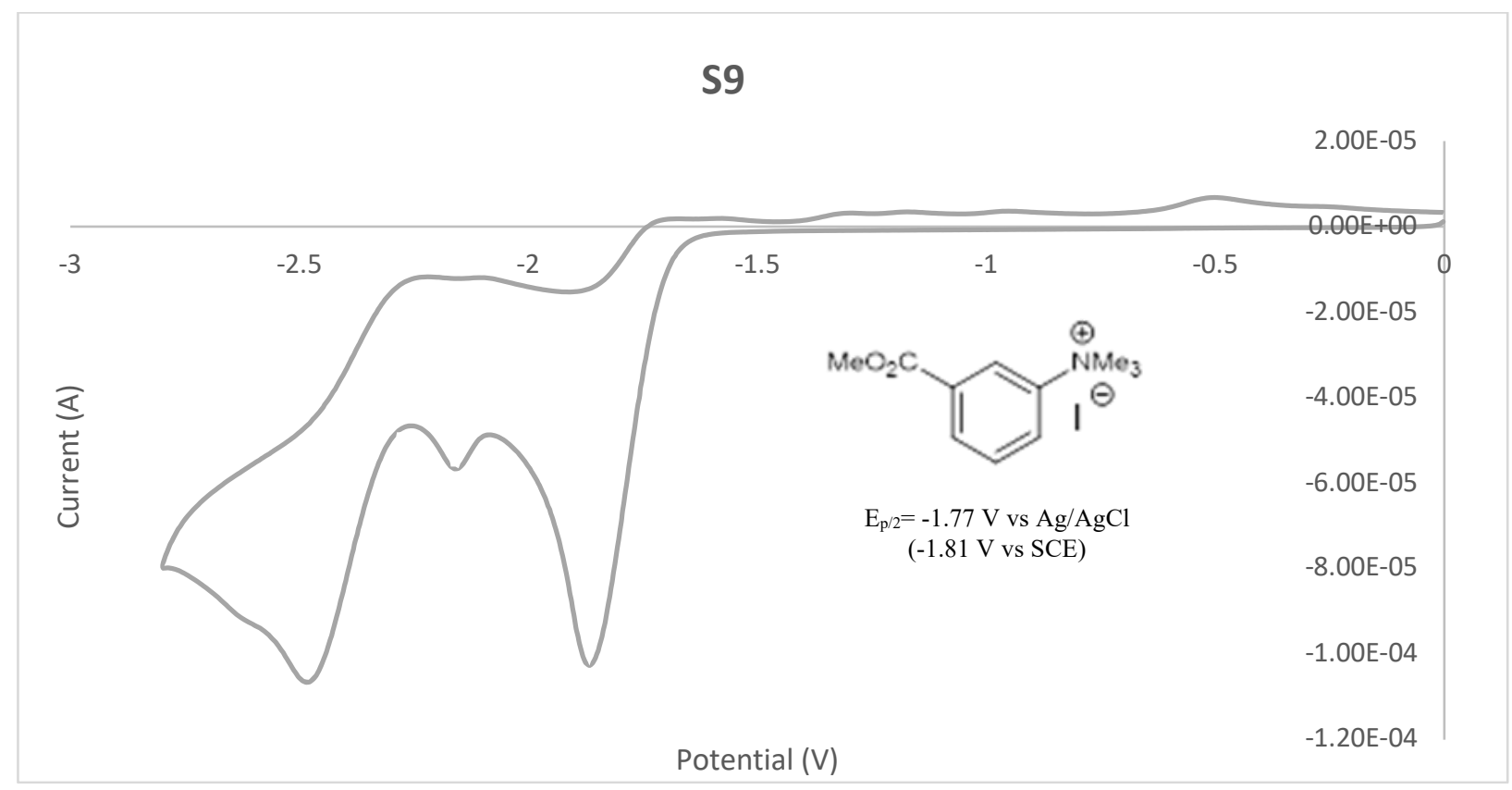

Figure S8. CV of 3-(methoxycarbonyl)-N,N,Ntrimethylbenzenaminium iodide (S9)

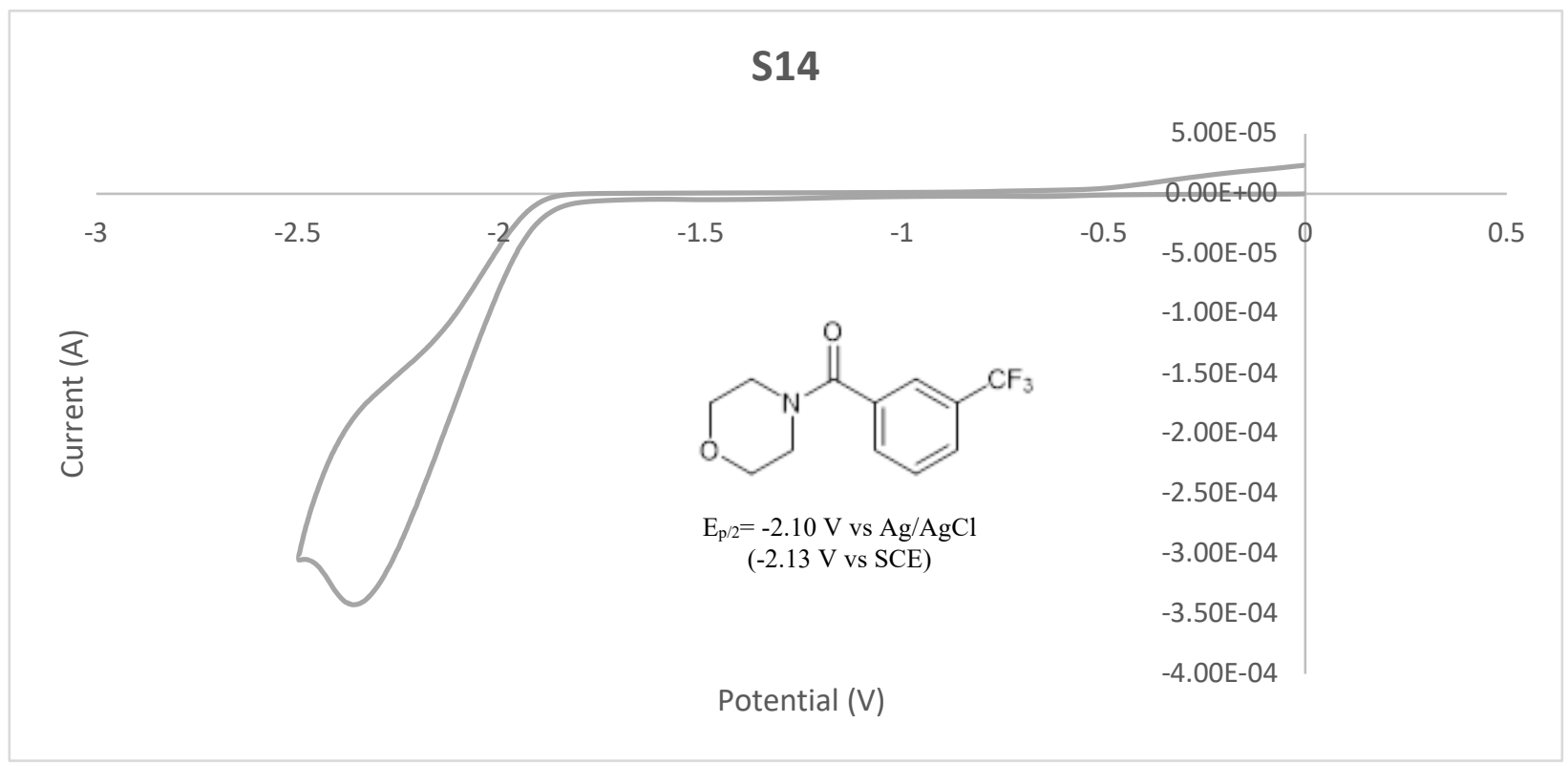

Figure S9. CV of morpholino(3-(trifluoromethyl)phenyl)methanone (S14) 


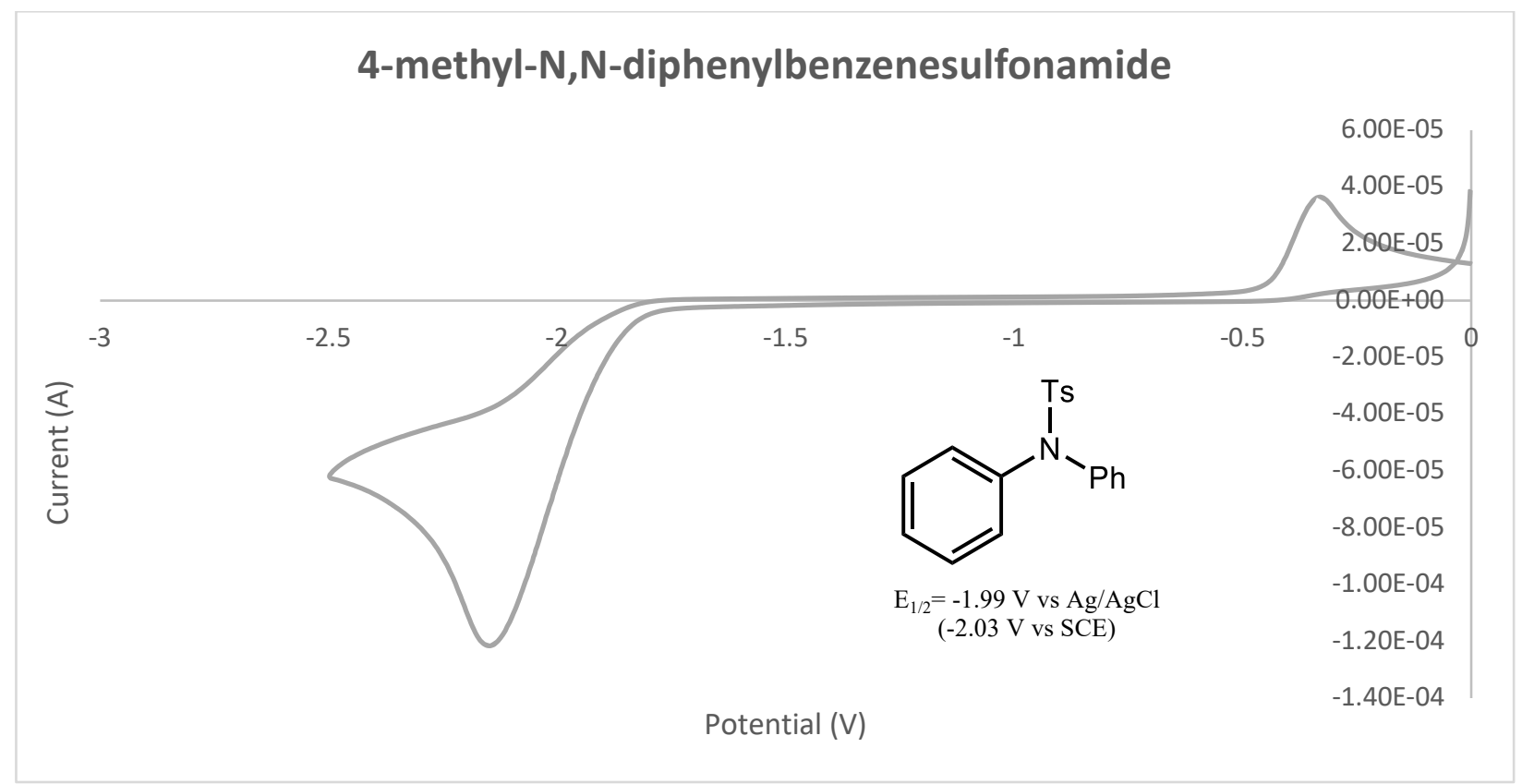

Figure S10. CV of 4-methyl-N,N-diphenylbenzenesulfonamide

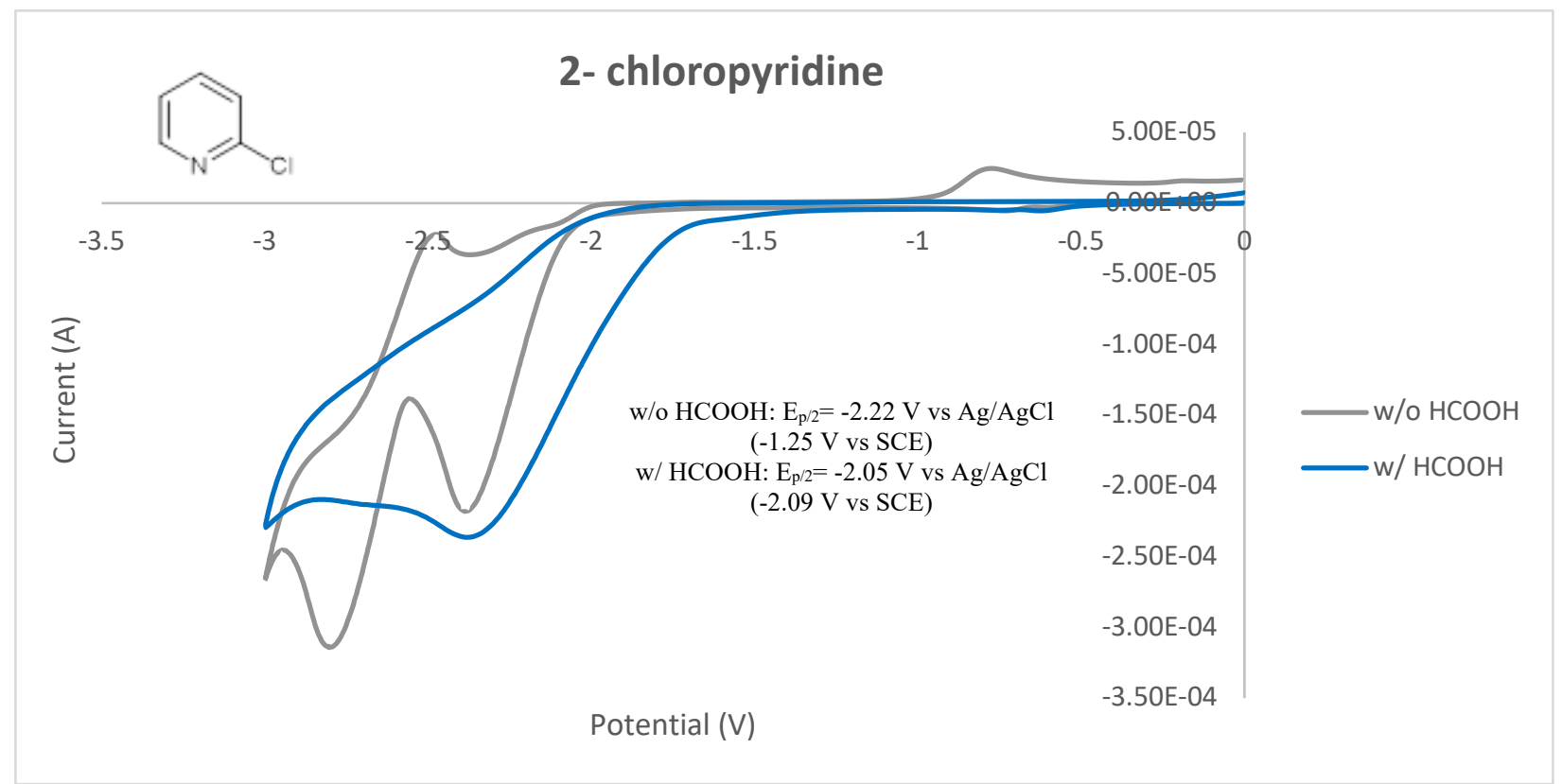

Figure S11. CV of 2-chloropyridine in the absence of formic acid (gray line) and in the presence of 5.0 eq. of formic acid (blue) 


\section{References}

1. Luo, J.; Zhang, J. "Donor-Acceptor Fluorophores for Visible-Light-Promoted Organic Synthesis: Photoredox/ Ni Dual Catalytic C $\left(\mathrm{sp}^{3}\right)-\mathrm{C}\left(\mathrm{sp}^{2}\right)$ Cross- Coupling." ACS Catal. 2016, 6, 873-877.

2. Kassir, A.; Ragab, S.; Nguyen, T.; Pouget, F.; Guillot, R.; Scherrmann, M.; Boddaert, T.; Aitken, D. "Synthetic Access to All Four Stereoisomers of Oxetin." J. Org. Chem., 2016, 81, 9983-9991.

3. Andaloussi, M.; Lindh, J.; Savmarker, J.; Sjoberg, P.; Larhed, M. Microwave-Promoted Palladium (II)-Catalyzed C-P Bond Formation by Using Arylboronic Acids or Aryltrifluoroborates. Chem. Eur. J., 2009, 15, 13069-13074.

4. Takise, R.; Itami, K,; Yamaguchi, J. "Cyanation of Phenol Derivatives with Aminoacetonitriles by Nickel Catalysis.” Org. Lett. 2016, 18, 4428-4431.

5. Xie, M.; Wang, Z. "Nickel-Catalyzed Cross-Coupling of Aryltrimethylammonium Iodides with Organozinc Reagents.” Angew. Chem. Int. Ed. 2011, 50, $4901-490$

6. Liu, C.; Ji, C.; Zhou, T.; Hong, X.; Szostak, M. "Decarbonylative Phosphorylation of Carboxylic Acids via Redox- Neutral Palladium Catalysis.” Org. Lett. 2019, 21, 9256 9261.

7. Naksomboon, K.; Poater, J.; Bickelhaupt M.; Fernadez-Ibanez, M. A. "para-Selective CH olefination of Aniline Derivatives via Pd/S,O-Ligand Catalysis.” J. Am. Chem. Soc. 2019, 141, 6719-6725.

8. MacKenzie, I. A.; Wang, L.; Onuska, N. P. R.; Williams, O. F.; Begam, K.; Moran, A. M.; Dunietz, B. D.; Nicewicz, D. A. "Discovery and Characterization of an Acridine Radical Photoreductant." Nature 2020, 580, 76-80.

9. Takise, R.; Itami, K,; Yamaguchi, J. "Cyanation of Phenol Derivatives with Aminoacetonitriles by Nickel Catalysis.” Org. Lett. 2016, 18, 4428-4431.

10. Bordi, S.; Starr, J. "Hydropyridylation of Olefins by Intramolecular Minisci Reaction." Org. Lett., 2017, 19, 2290-2293

11. Peng, Z.; Wong, J.; Hansen, E.; Dermenci, A.; Clarke, H. "Development of a Concise, Asymmetric Synthesis of a Smoothened Receptor (SMO) Inhibitor: Enzymatic Transamination of a 4-Piperidinone with Dynamic Kinetic Resolution." Org. Lett., 2014, $16,860-863$. 
12. Huang, H.; Belloti, P.; Pfluger, P.; Schwarz, L.; Heidrich, B.; Glorius, F. ThreeComponent, Interrupted Radical Heck/Allylic Substitution Cascade Involving Unactivated Alkyl Bromides. J. Am. Chem. Soc., 2020, 142, 10173-10183.

13. Wang, H.; Jui, N. "Catalytic Defluoroalkylation of Trifluoromethylaromatics with Unactivated Alkenes.” J. Am. Chem. Soc. 2018, 140, 1, 163-166

14. Cheng, R.; Shi, Z.; Ding, W.; Liu, Z.; Jin, H.; Yu, X.; Wang, S. Facile Synthesis of alphabeta unsaturated esters through a one-pot copper catalyzed aerobic oxidation-Wittig reaction. Tett. Lett., 2018, 59, 14-17.

15. 3. Li, W.; Tang, J.; Li, S.; Zheng, X.; Yuan, M.; Xu, B.; Jiang, W.; Fu, H.; Li, R.; Chen, $\mathrm{H}$. Stereodivergent Synthesis of Alkenylpyridines via $\mathrm{Pd} / \mathrm{Cu}$ Catalyzed C-H Alkenylation of Pyridinium Salts with Alkynes. Org. Lett., 2020, 22, 7814-7819.

16. Liu, Z.; Dong, N.; Xu, M.; Sum, Z.; Tu, T. "Mild Negishi Cross-Coupling Reactions Catalyzed by Acenaphtoimidazolyidene Palladium Complexes at Low Catalyst Loadings.” J. Org. Chem., 2013, 78, 7436-7444.

17. Mader, P.; Bartholomaus, R.; Nicolussi, S.; Baumann, A.; Weis, M.; Chicca, A.; Rau, M.; Simao, A.; Gertsch, J.; Altmann, K. "Synthesis and Biological Evaluation of Endocannabinoid Uptake Inhibitors Derived from WOBE437. ” ChemMedChem., 2021, $16,145-154$.

18. Kim, S.; Goldfogel, M.; Gilbert, M.; Weix, D. "Nickel-Catalyzed Cross-Electrophile Coupling of Aryl Chlorides with Primary Alkyl Chlorides." J. Am. Chem. Soc., 2020, 142, 9902-9907.

19. Ackermann, L.; Punji, B.; Song, W. "User-Friendly [(Diglyme)NiBr2]-Catalyzed Direct Alkylations of Heteroarenes with Unactivated Alkyl Halides through C-H Bond Cleavages." Adv. Synth. Catal., 2011, 353, 3325-3329.

20. Boyington, A.; Riu, M.; Jui, N. "Anti-Markovnikov Hydroarylation of Unactivated Olefins via Pyridyl Radical Intermediates.” J. Am. Chem. Soc. 2017, 139, 19, 6582-6585.

21. Hara, N.; Saito, T.; Semba, K.; Kuriakose, N.; Zheng, H.; Sakaki, S.; Nako, Y. "Rhodium Complexes Bearing PAIP Pincer Ligands.” J. Am. Chem. Soc. 2018, 140, 7070-7073.

22. Kondolff, I.; Doucet, H.; Santelli, M. “Tetraphosphine/palladium catalysed Suzuki crosscoupling reactions of aryl halides with alkylboronic acids." Tetrahedon Lett. 2004, 60, 3813-3818. 
23. Cassani, C.; Bergonzini, G.; Wallentin, C. "Photocatalytic Decarboxylative Reduction of Carboxylic Acids and Its Application in Asymmetric Synthesis." Org. Lett., 2014, 16, 4228-4231.

24. Cassani, C.; Bergonzini, G.; Wallentin, C. Photocatalytic Decarboxylative Reduction of Carboxylic Acids and Its Application in Asymmetric Synthesis. Org. Lett., 2014, 16, 4228-4231.

25. Wang, T.; Sang, S.; Liu, L.; Qiao, H.; Gao, Y.; Zhao, Y. "Experimental and Theoretical Study on Palladium- Catalyzed C-P Bond Formation via Direct Coupling of Triarylbismuths with P(O)-H Compounds.” J. Org. Chem. 2014, 79, 608-617.

26. Xia, Q.; Liu, X.; Zhang, Y.; Chen, C.; Chen, W. “Copper- Catalyzed N-Methylation of Amides and O-Methylation of Carboxylic Acids by Using Peroxides as the Methylating Reagents." Org. Lett. 2013, 15, 3326-3329.

27. Suarez-Pantiga, S.; Hernandez-Ruiz, R.; Virumbrales, C.; Pedrosa, M.; Sanz, R. "Reductive Molybdenum-Catalyzed Direct Amination of Boronic Acids with Nitro Compounds." Angew. Chem. Int. Ed. 2019, 58, 2129-2133.

28. Z.; Sun, Q.; Xia, C.; Sun, W. " $\mathrm{CO}_{2}$ as a $\mathrm{C} 1$ Source: $\mathrm{B}\left(\mathrm{C}_{6} \mathrm{~F}_{5}\right)_{3}$-Catalyzed Cyclization of $o$ Phenylene-diamines To Construct Benzimidazoles in the Presence of Hydrosilane." Org. Lett. 2016, 18, 6316-6319.

29. Bonfio, C.; Caumes, C.; Duffy, C.; Patel, B.; Percivalle, C.; Tsnakopoulou, M.; Sutherland, J. "Length-Selective Synthesis of Acylglycerol-Phosphates through EnergyDissipative Cycling." J. Am. Chem. Soc. 2019, 141, 3934-3939

30. Houjeiry, T.; Poe, S.; McQuade, T. "Synthesis of Optically Active 4-Substituted 2Cyclohexenones.” Org. Lett. 2012, 14, 4394-4397

31. Tamang, S.; Bedi, D.; Shafiei-Haghighi, S.; Smith, C Crawford, C.; Findlater, M. "Cobalt-Catalyzed Hydroboration of Alkenes, Aldehydes, and Ketones." Org. Lett. 2018, 20, 6695-6700.

32. Li, W.; Tang, J.; Li, S.; Zheng, X.; Yuan, M.; Xu, B.; Jiang, W.; Fu, H.; Li, R.; Chen, H. Stereodivergent Synthesis of Akenylpyridines via $\mathrm{Pd} / \mathrm{Cu}$ Catalyzed C-H Alkenylation of Pyridinium Salts with Alkynes. Org. Lett., 2020, 22, 7814-7819.

33. Hager, D.; MacMillan, D. "Activation of C-H Bonds via the Merger of Photoredox and Organocatalysis: A Coupling of Benzylic Ethers with Schiff Bases.” J. Am. Chem. Soc. 2014, 136, 16986-16989 
34. Cismesia, M.; Yoon, T. "Characterizing chain processes in visible light photoredox catalysis.” Chem. Sci., 2015, 6, 5426-5434.

35. Zhang, Z.; Sun, Q.; Xia, C.; Sun, W. $\mathrm{CO}_{2}$ as a C1 Source: B(C6F5)3-Catalyzed Cyclization of o-Phenylene-diamines to Construct Benzimidazoles in the Presence of Hydrosilane. Org. Lett., 2016, 18, 6316-6319.

36. M. J. Frisch, G. W. Trucks, H. B. Schlegel, G. E. Scuseria, M. A. Robb, J. R. Cheeseman, G. Scalmani, V. Barone, G. A. Petersson, H. Nakatsuji, X. Li, M. Caricato, A. Marenich, J. Bloino, B. G. Janesko, R. Gomperts, B. Mennucci, H. P. Hratchian, J. V. Ortiz, A. F. Izmaylov, J. L. Sonnenberg, D. Williams-Young, F. Ding, F. Lipparini, F. Egidi, J. Goings, B. Peng, A. Petrone, T. Henderson, D. Ranasinghe, V. G. Zakrzewski, J. Gao, N. Rega, G. Zheng, W. Liang, M. Hada, M. Ehara, K. Toyota, R. Fukuda, J. Hasegawa, M. Ishida, T. Nakajima, Y. Honda, O. Kitao, H. Nakai, T. Vreven, K. Throssell, J. A. Montgomery, Jr., J. E. Peralta, F. Ogliaro, M. Bearpark, J. J. Heyd, E. Brothers, K. N. Kudin, V. N. Staroverov, T. Keith, R. Kobayashi, J. Normand, K. Raghavachari, A. Rendell, J. C. Burant, S. S. Iyengar, J. Tomasi, M. Cossi, J. M. Millam, M. Klene, C. Adamo, R. Cammi, J. W. Ochterski, R. L. Martin, K. Morokuma, O. Farkas, J. B. Foresman, and D. J. Fox, Gaussian, Inc., Wallingford CT, 2016.

37. (a) Lee, C.; Yang, W.; Parr, R. G. Phys. Rev. B, 1988, 37, 785-789. (b) Becke, A. D. J. Chem. Phys. 1993, 98, 5648-5652.

38. Roth, H. G.; Romero, N. A.; Nicewicz, D. A. "Experimental and Calculated Electrochemical Potentials of Common Organic Molecules for Applications to SingleElectron Redox Chemistry." Synlett 2016, 27, 714-723.

39. Isse, A. A.; Gennaro, A. "Absolute Potential of the Standard Hydrogen Electrode and the Problem of Interconversion of Potentials in Different Solvents." J. Phys. Chem. B 2010, 114, 7894-7899. 


\section{NMR Spectra}

\section{3-cyano-N,N,N-trimethylbenzenaminium iodide (S5):}

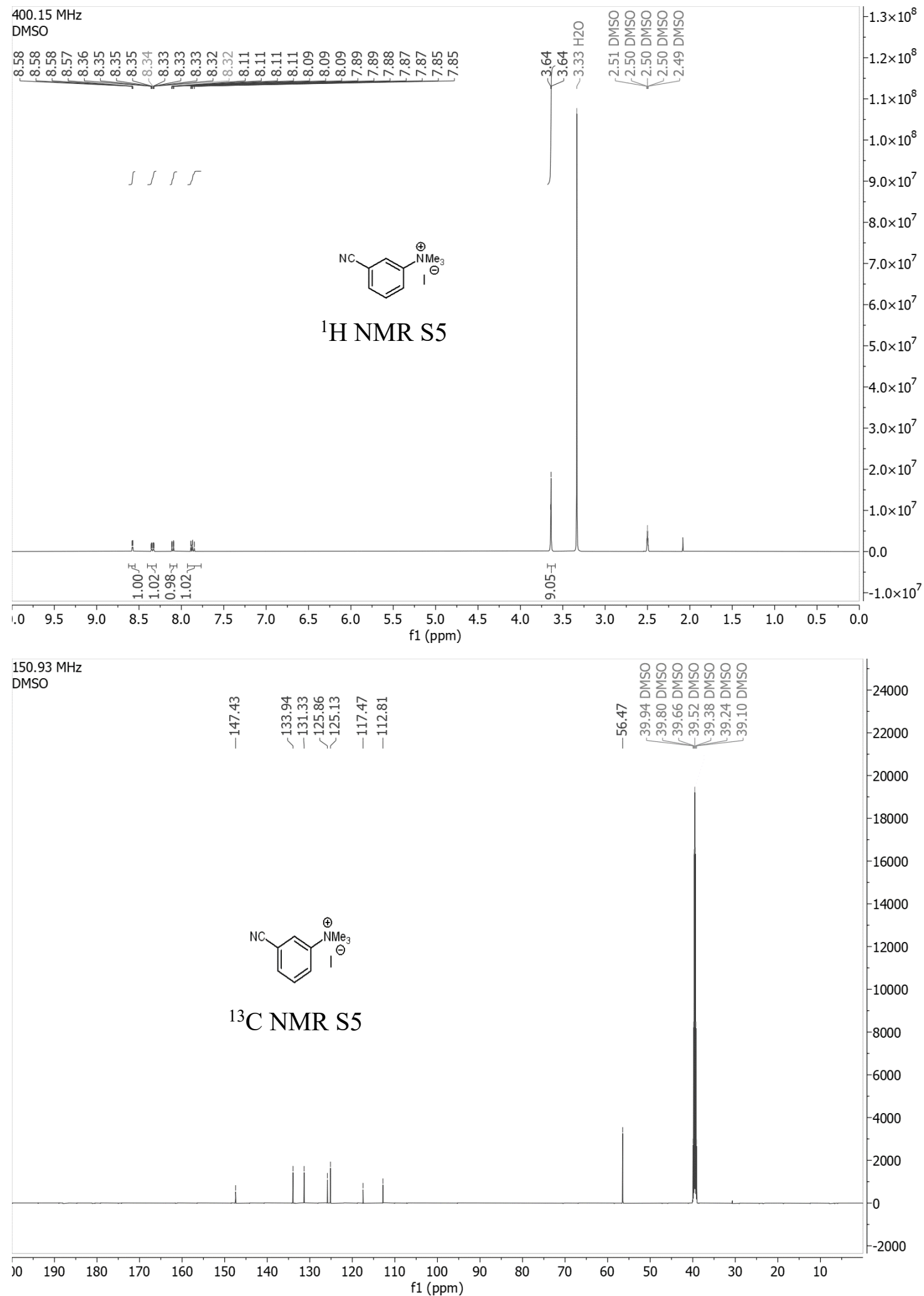




\section{diethyl (3-(dimethylamino)phenyl)phosphonate (S7):}

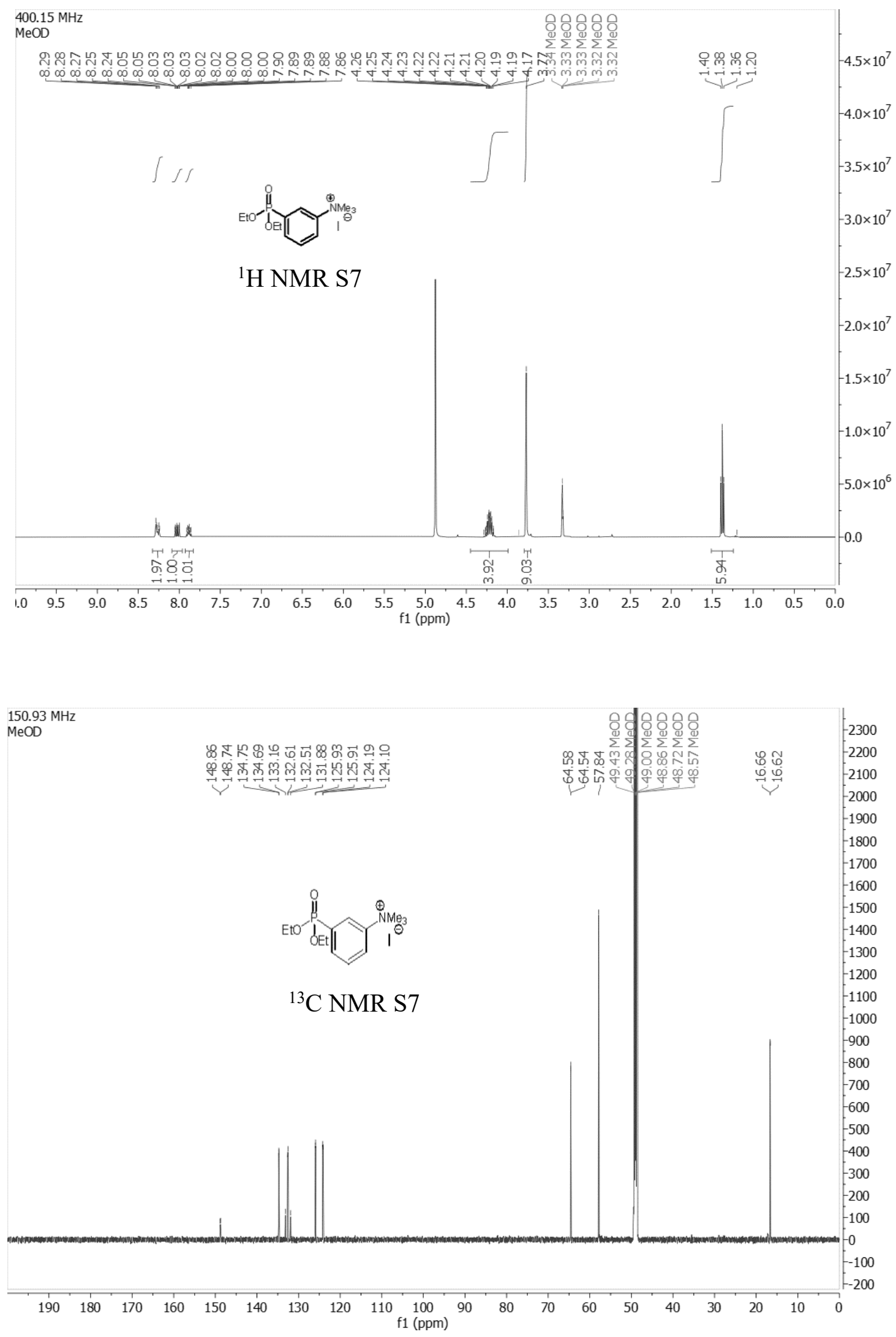




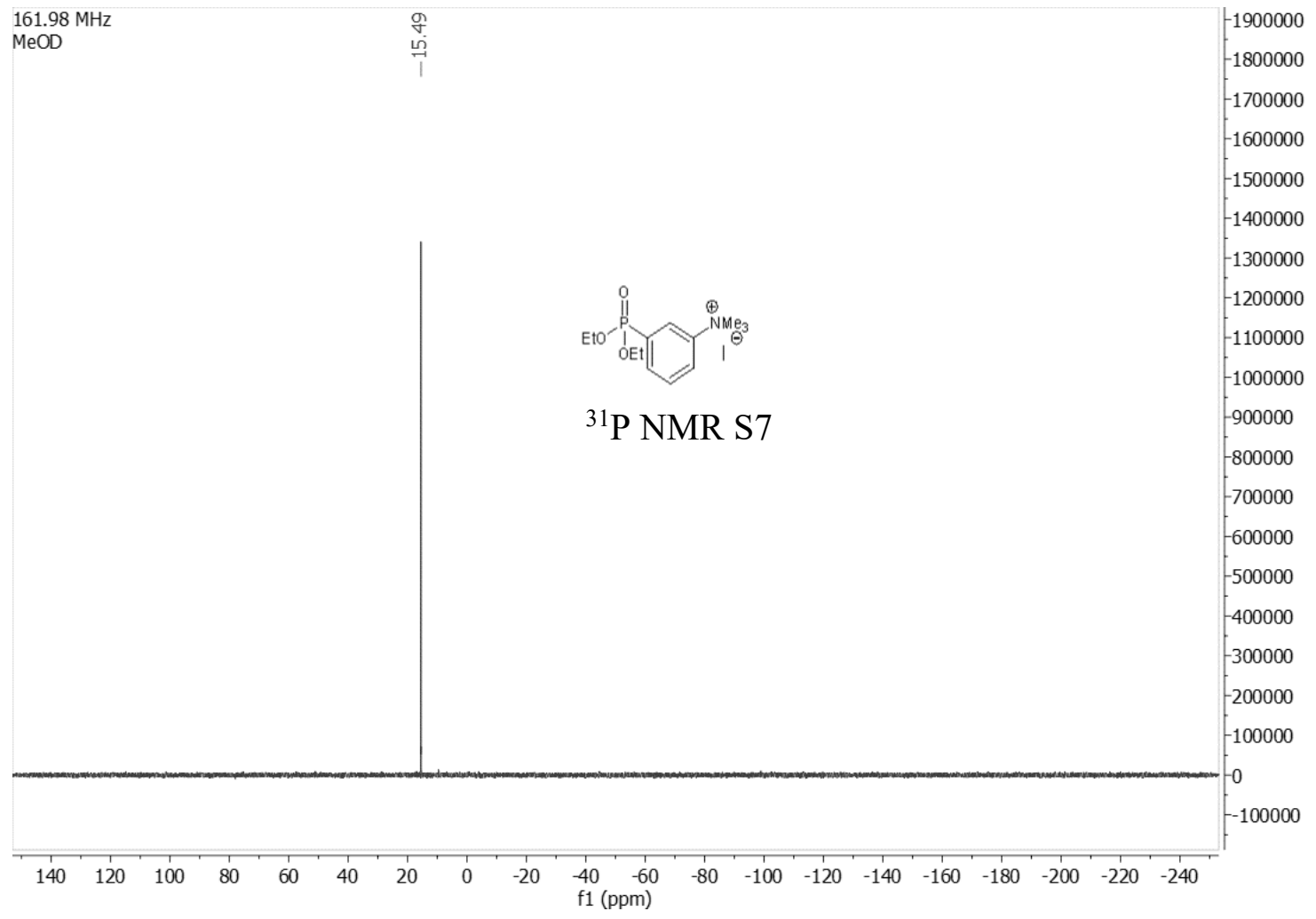




\section{3-(methoxycarbonyl)-N,N,N-trimethylbenzenaminium iodide (S9):}

$399.80 \mathrm{MHz}$

DMSO

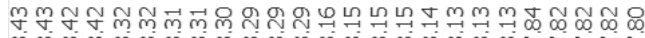

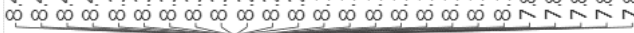

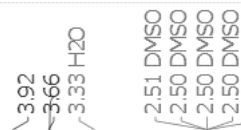

$\iiint \quad \int$

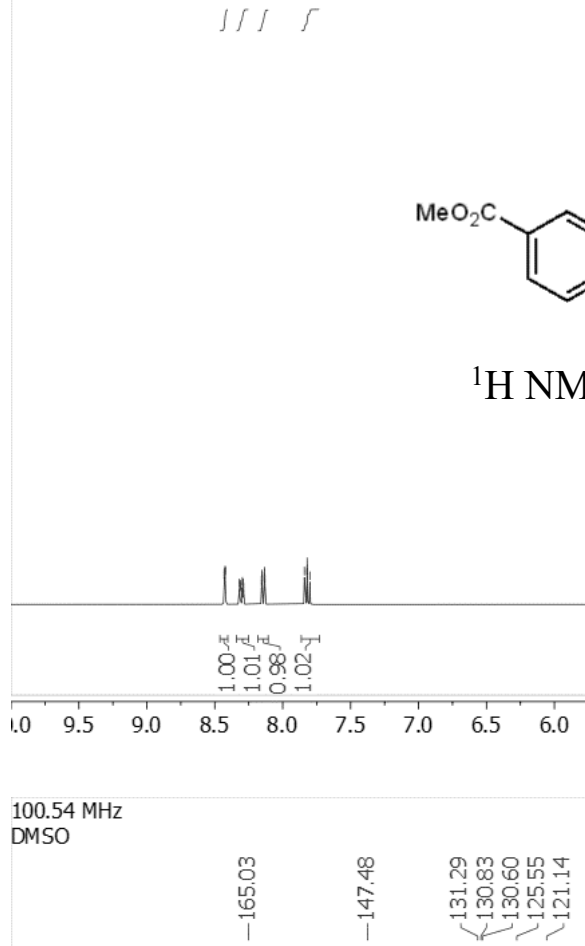

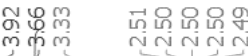

120000

110000

100000

90000

80000

70000

60000

50000

40000

30000

20000

10000

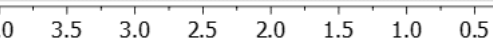
5.0
$\mathrm{f} 1(\mathrm{ppm})$

4.0

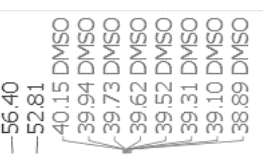

¿SOUNO

240000

$-230000$

$-220000$

$-210000$

$-200000$

$-190000$

$-180000$

$-170000$

$-160000$<smiles>COC(=O)c1cccc(N(C)C)c1</smiles>

$-150000$

$-140000$

$-130000$

$-120000$

$-110000$

$-100000$

${ }^{13} \mathrm{C}$ NMR S9

$-90000$

$-80000$

$-70000$

$-60000$

$-50000$

$-40000$

$-30000$

$-20000$

$-10000$

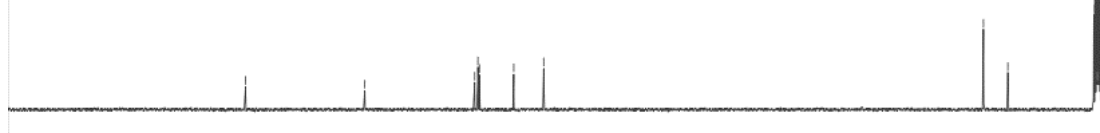

$-0$ 


\section{(E)-N-phenethylbut-2-enamide (S15):}
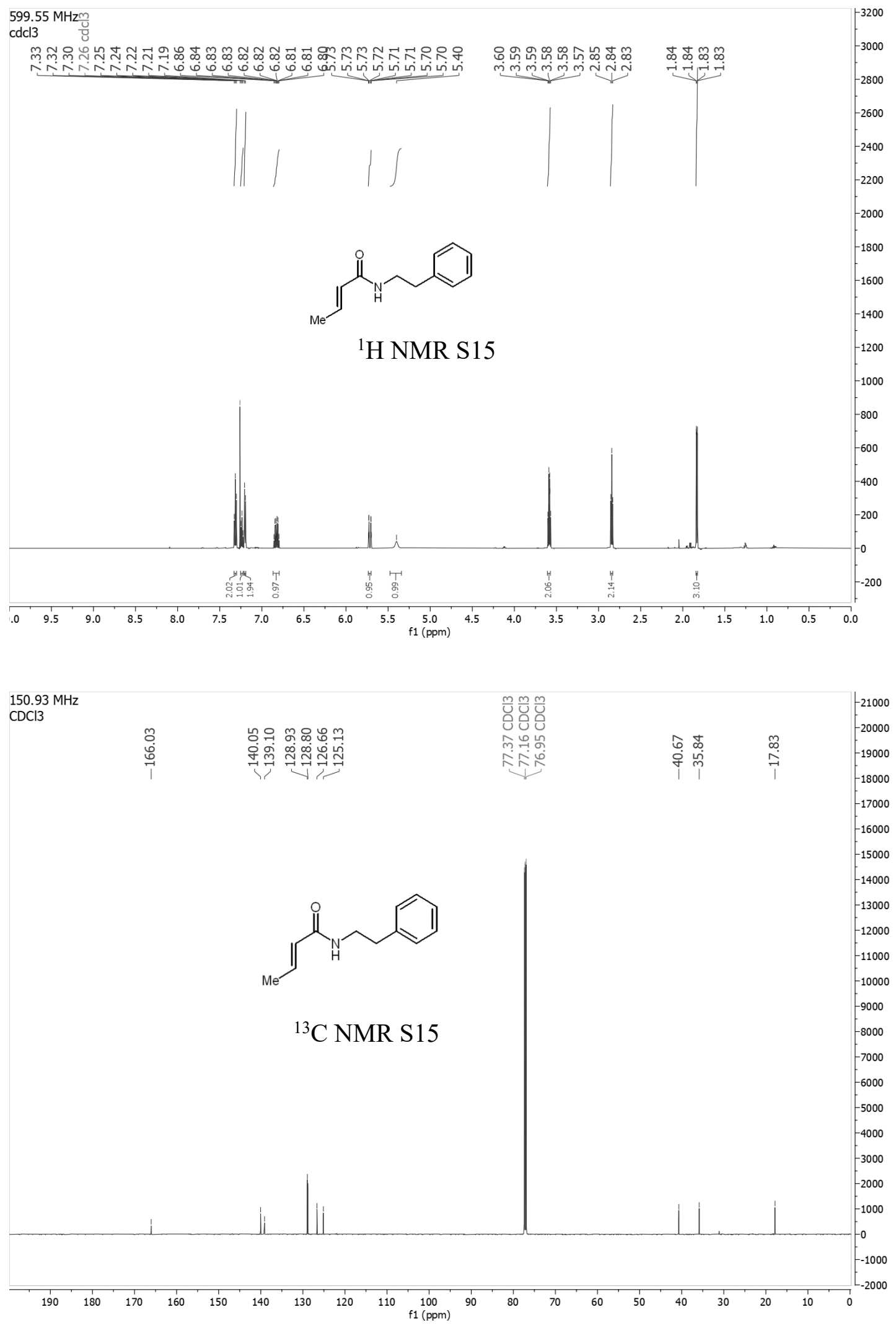


\section{(E)-1-cyclopropyl-6-phenylhex-1-en-3-one (S17):}

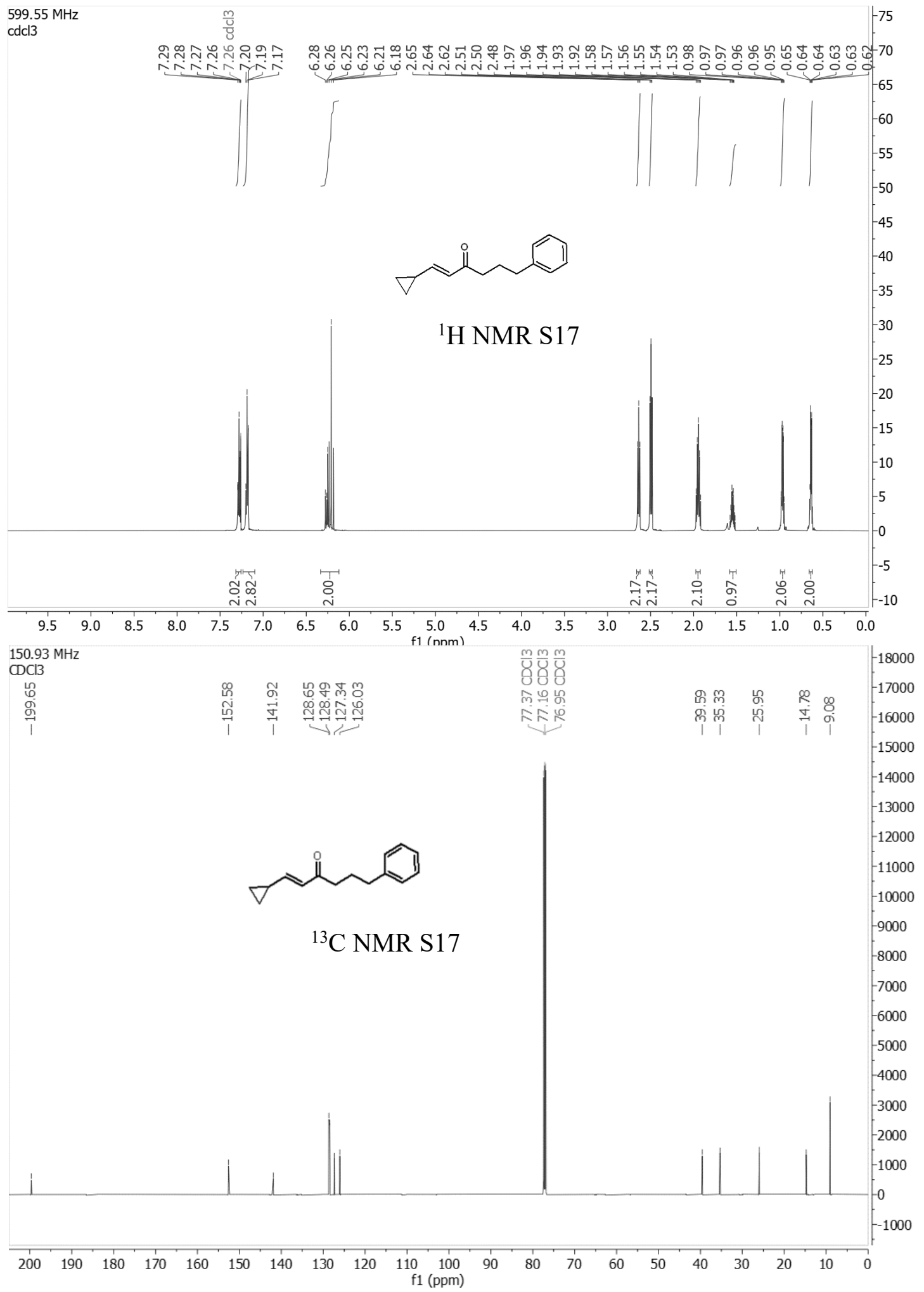




\section{4-(4-hydroxybutyl)benzonitrile (2):}

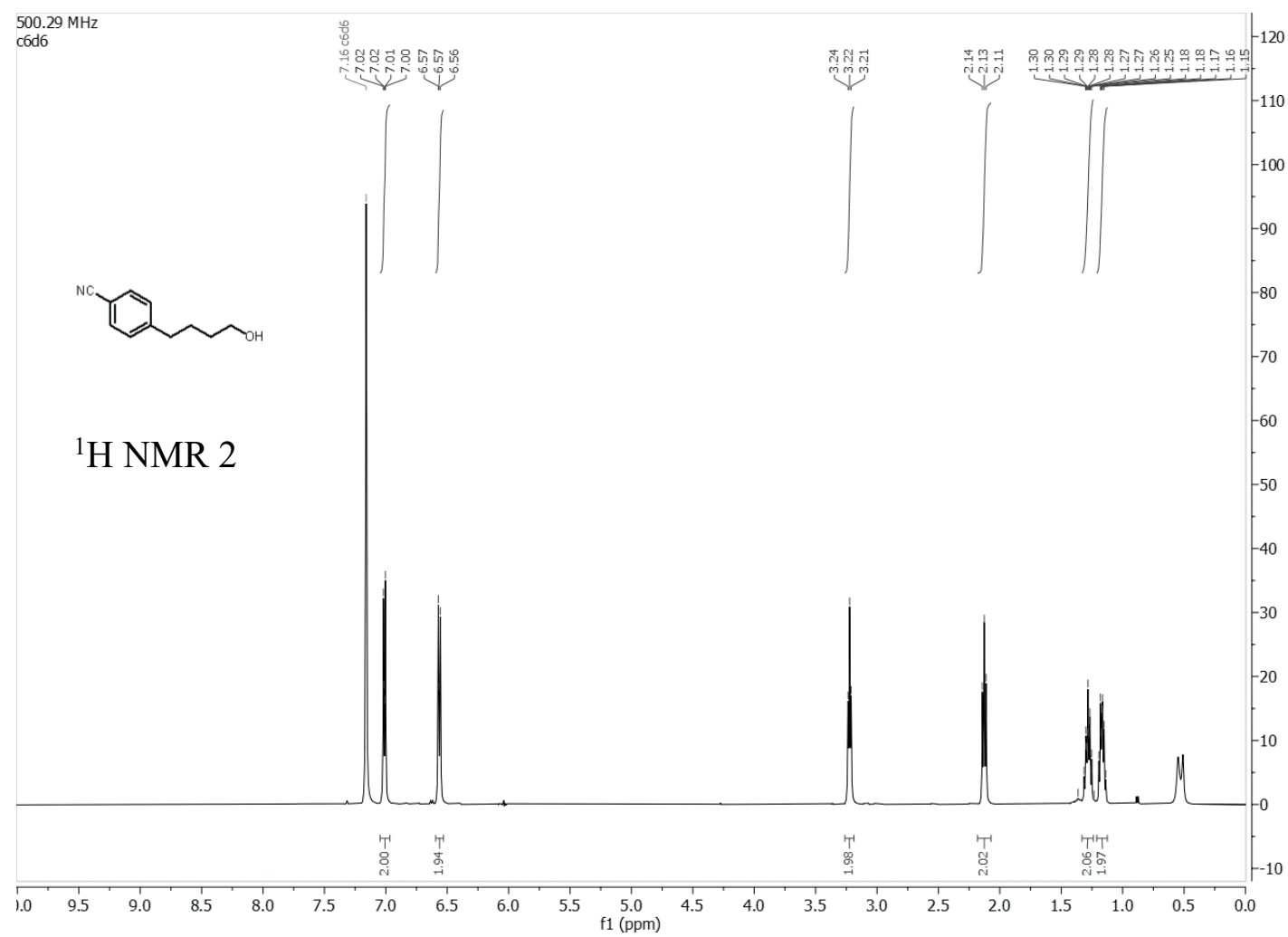

$100.54 \mathrm{MHz}$
$0 \mathrm{Md} 3$

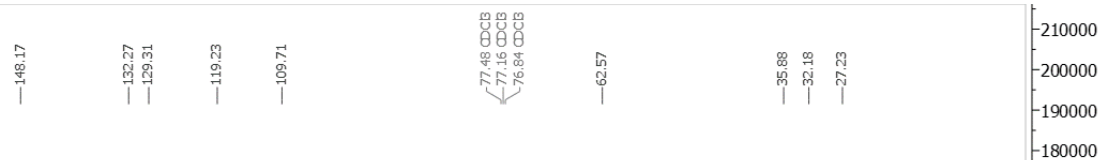

NC)

$-170000$

${ }^{13} \mathrm{C}$ NMR 2

$-160000$

$-150000$

$-140000$

$-130000$

$-120000$

$-110000$

$-100000$

$-90000$

$-80000$

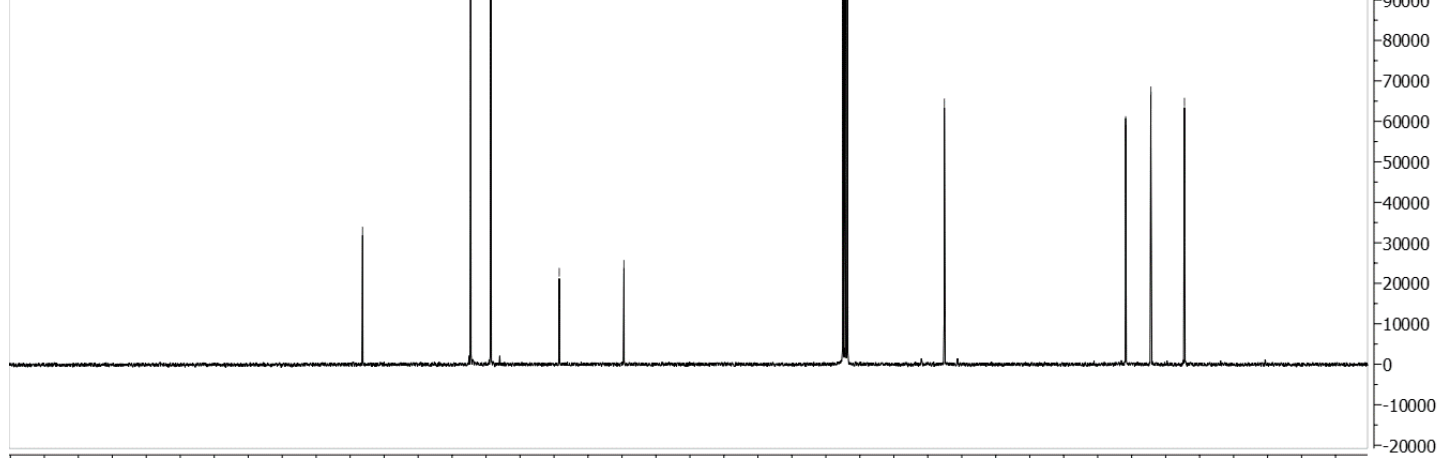

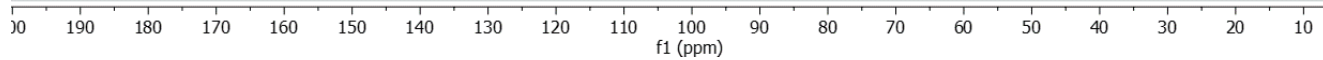




\section{4-(6-chlorohexyl)benzonitrile (3):}

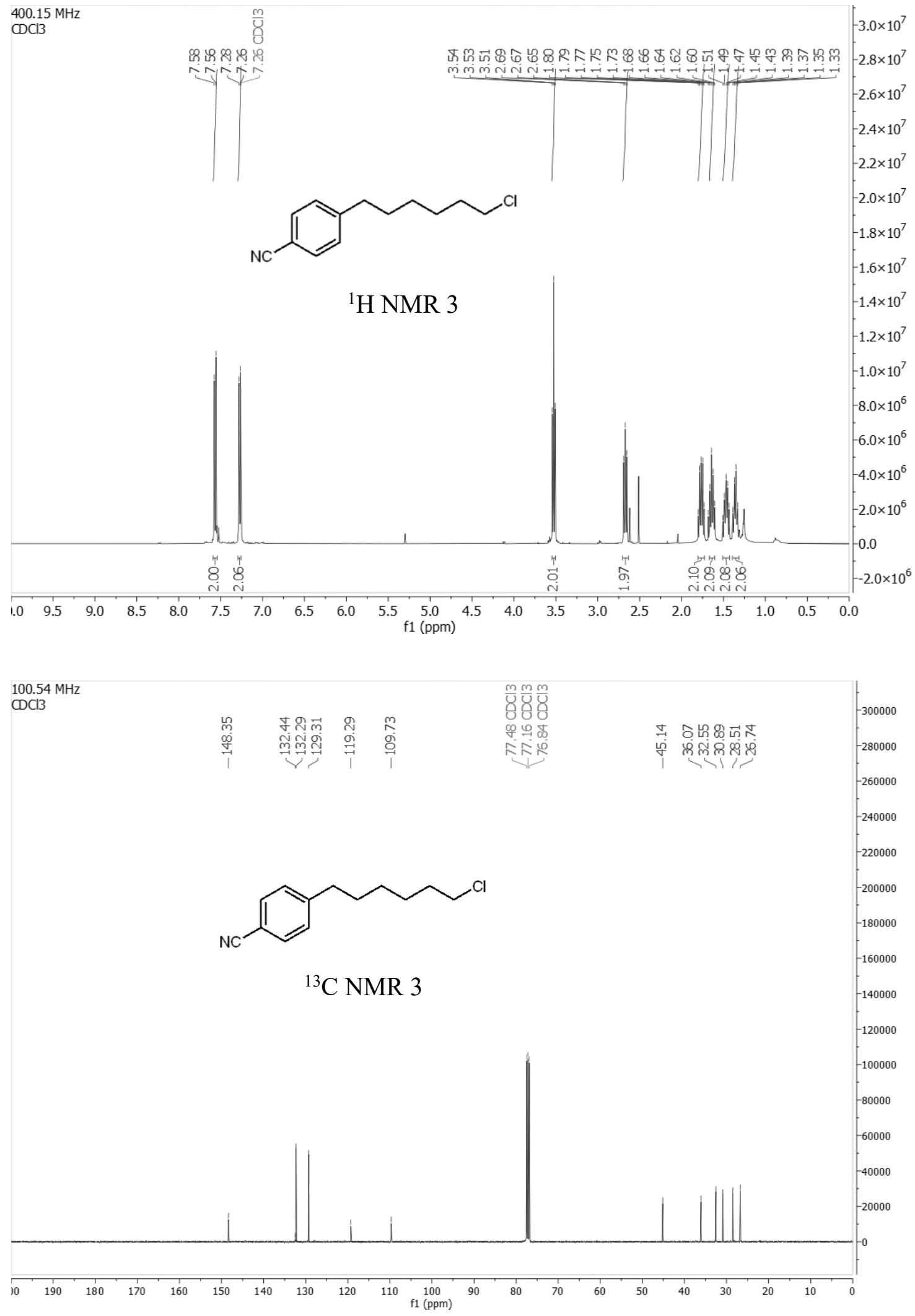




\section{4-(3-methylbutan-2-yl)benzonitrile) (4):}
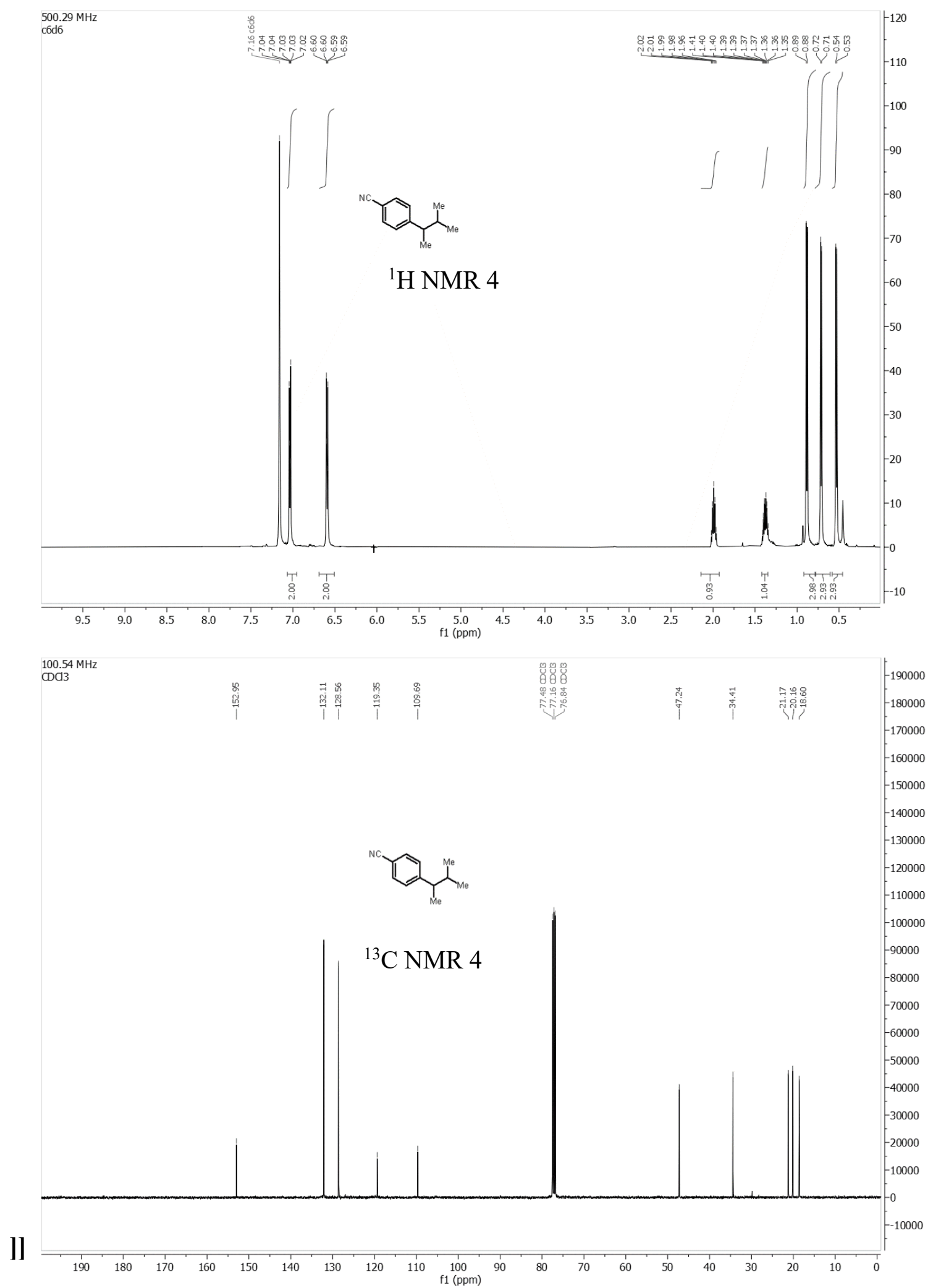


\section{4-(2-ethoxyethyl)benzonitrile (5):}

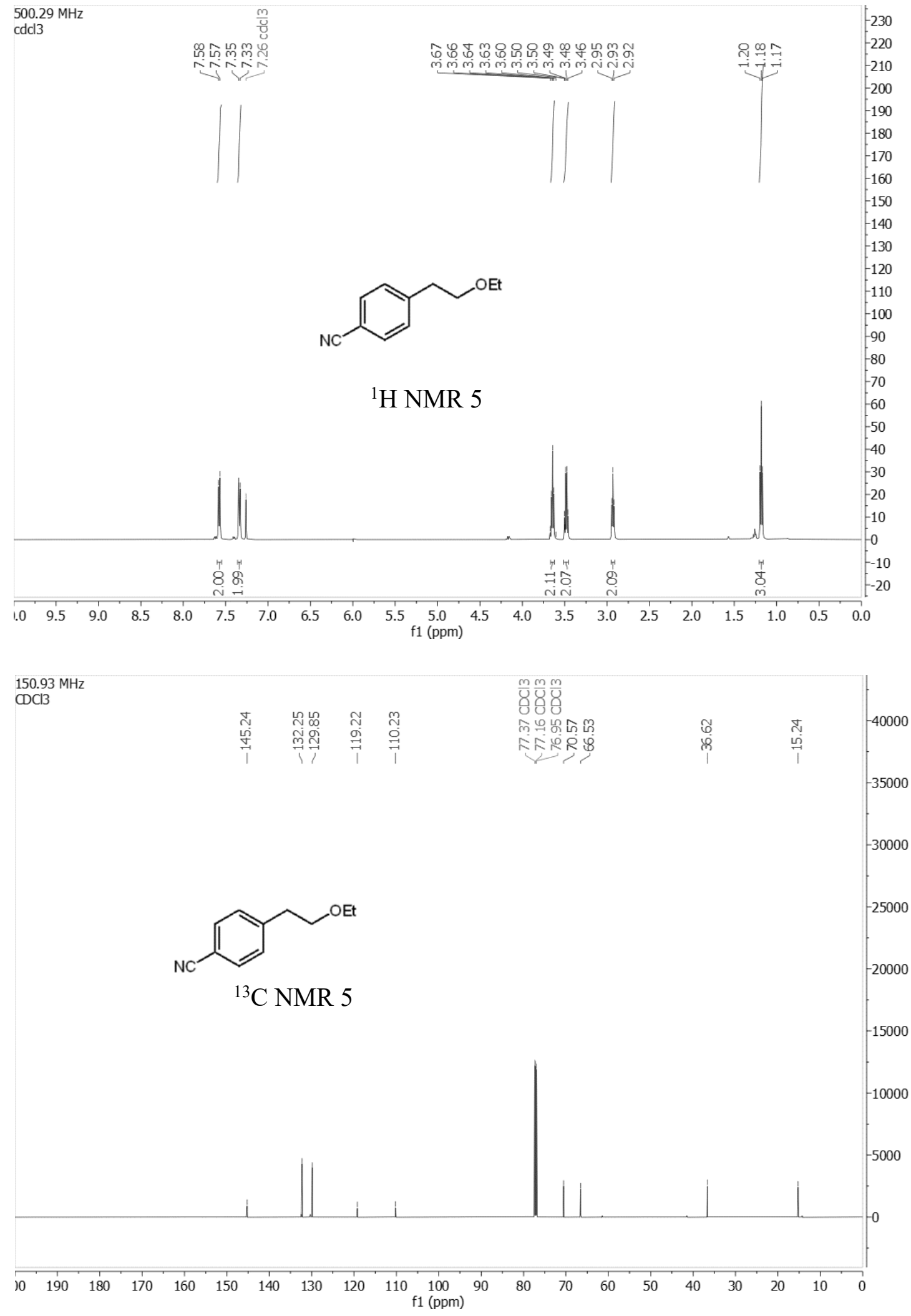



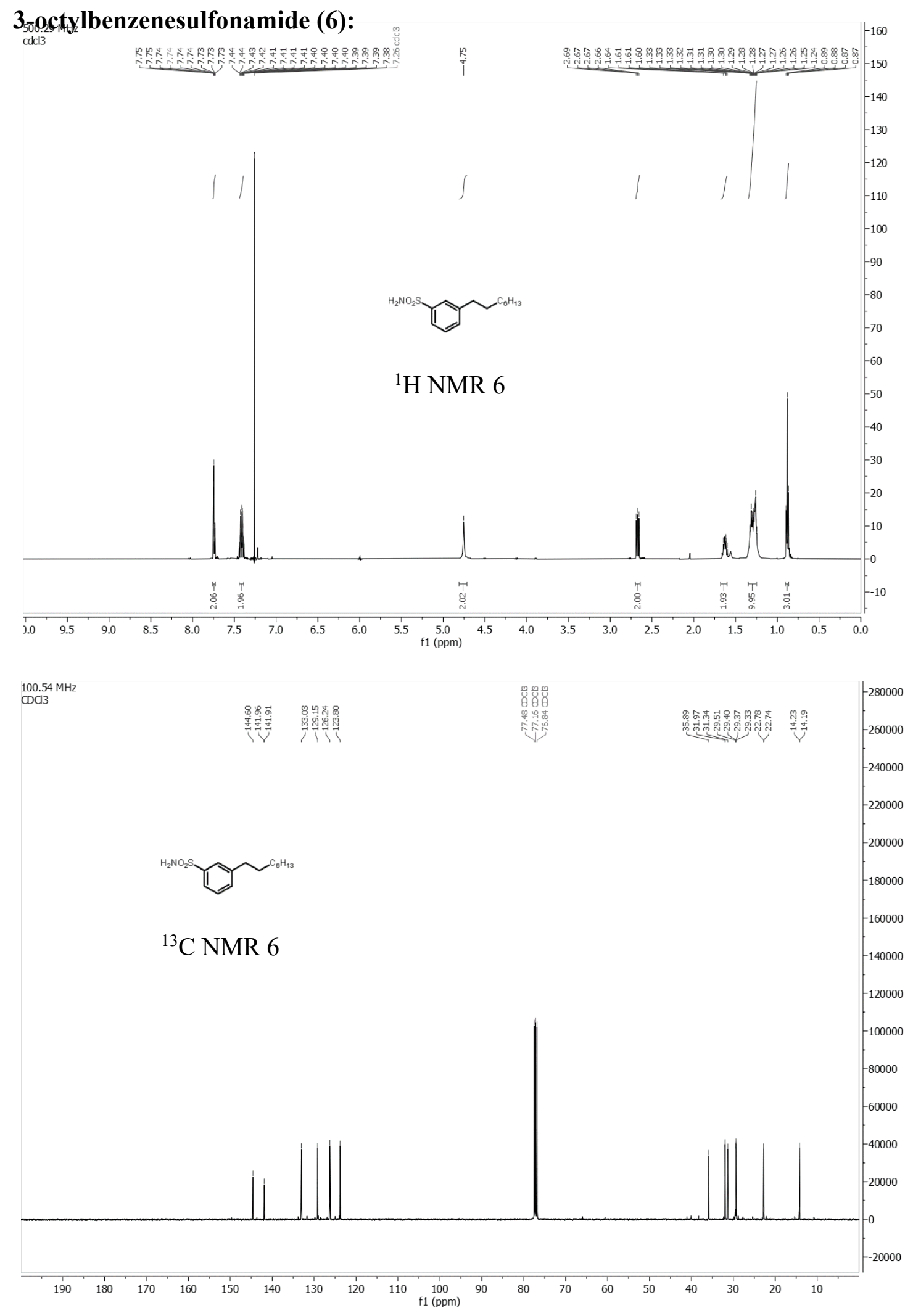


\section{Diethyl (4-octlyphenyl)phosphonate (7):}
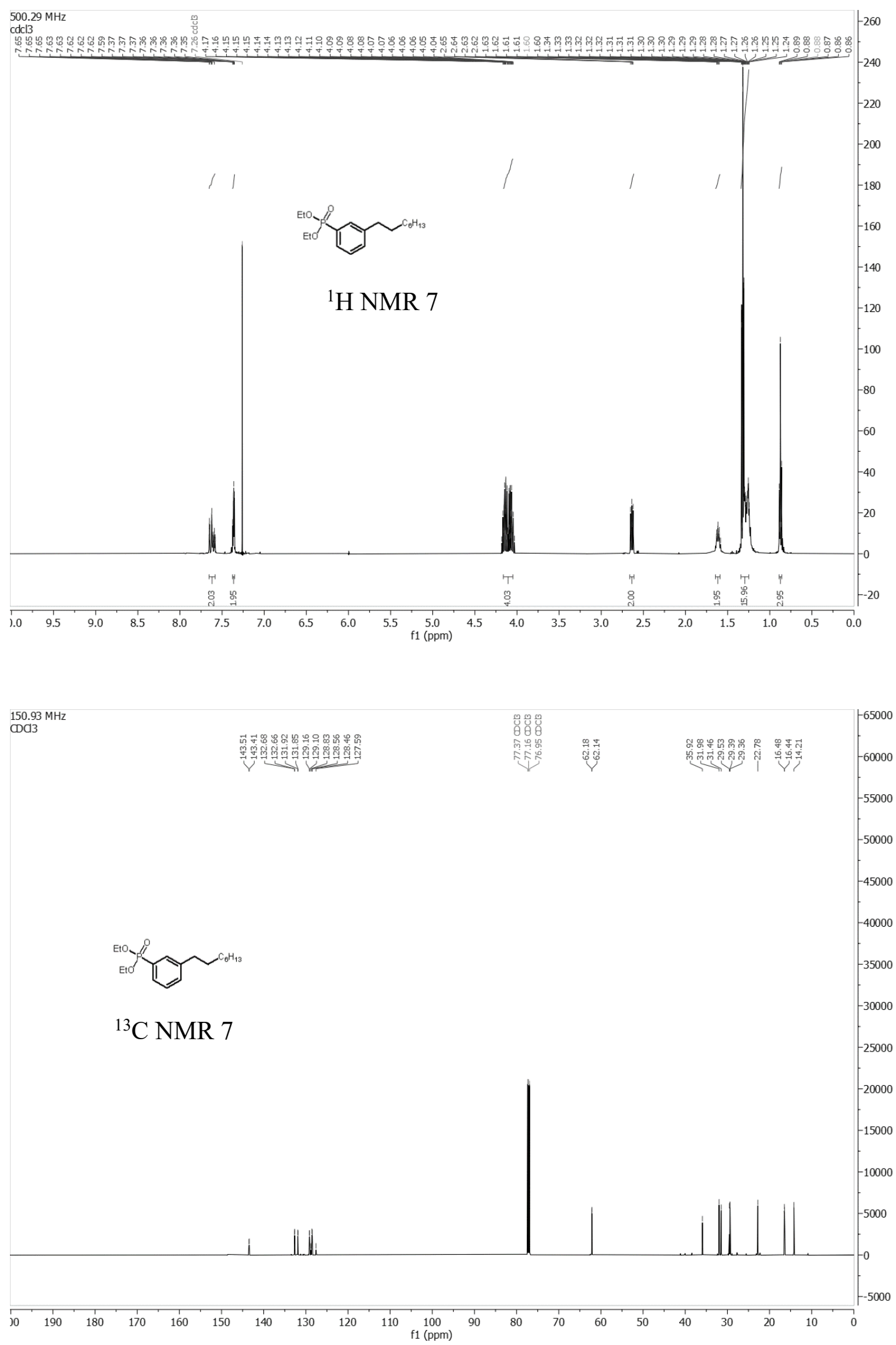


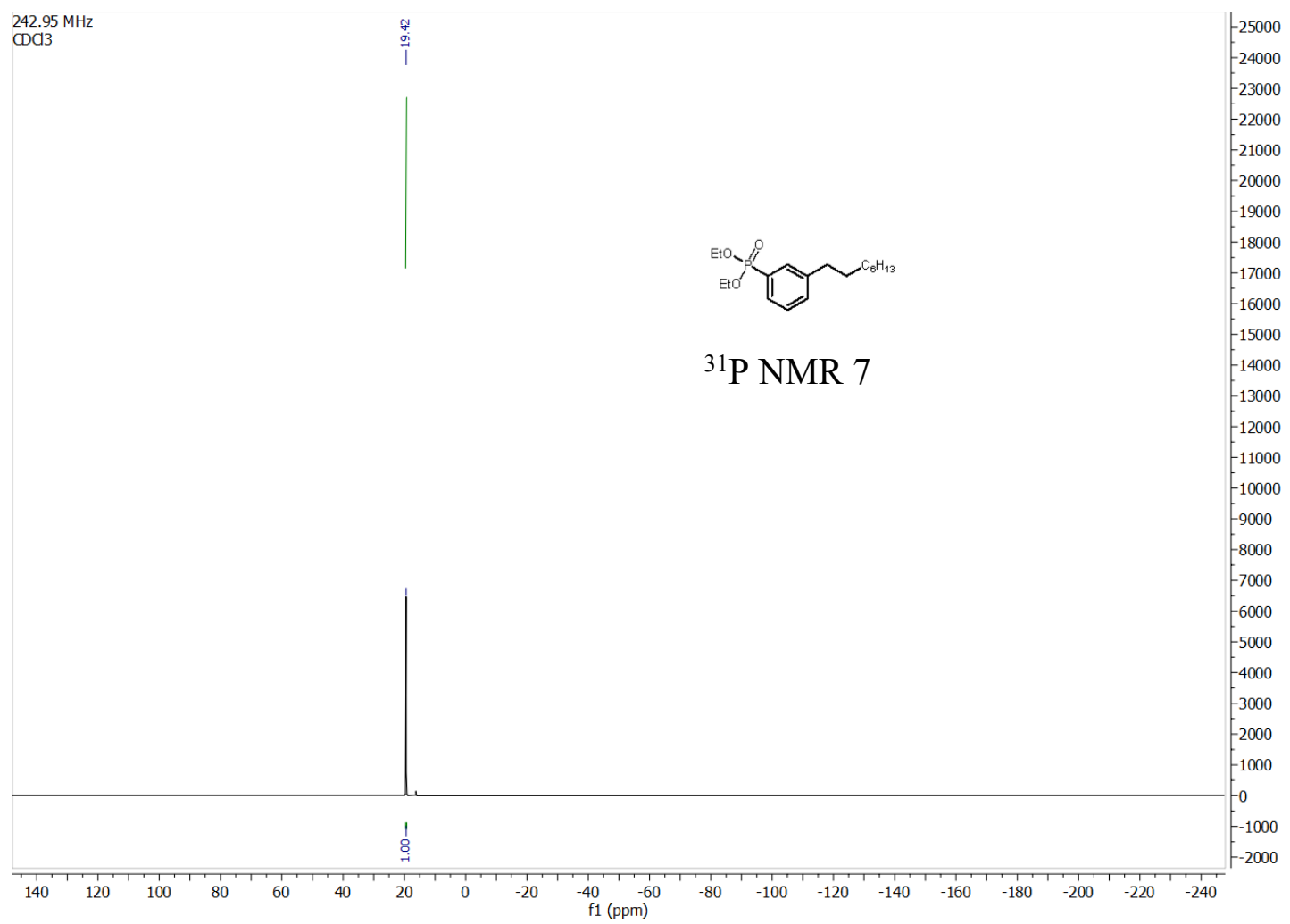


Methyl-2-octylbenzoate (10):
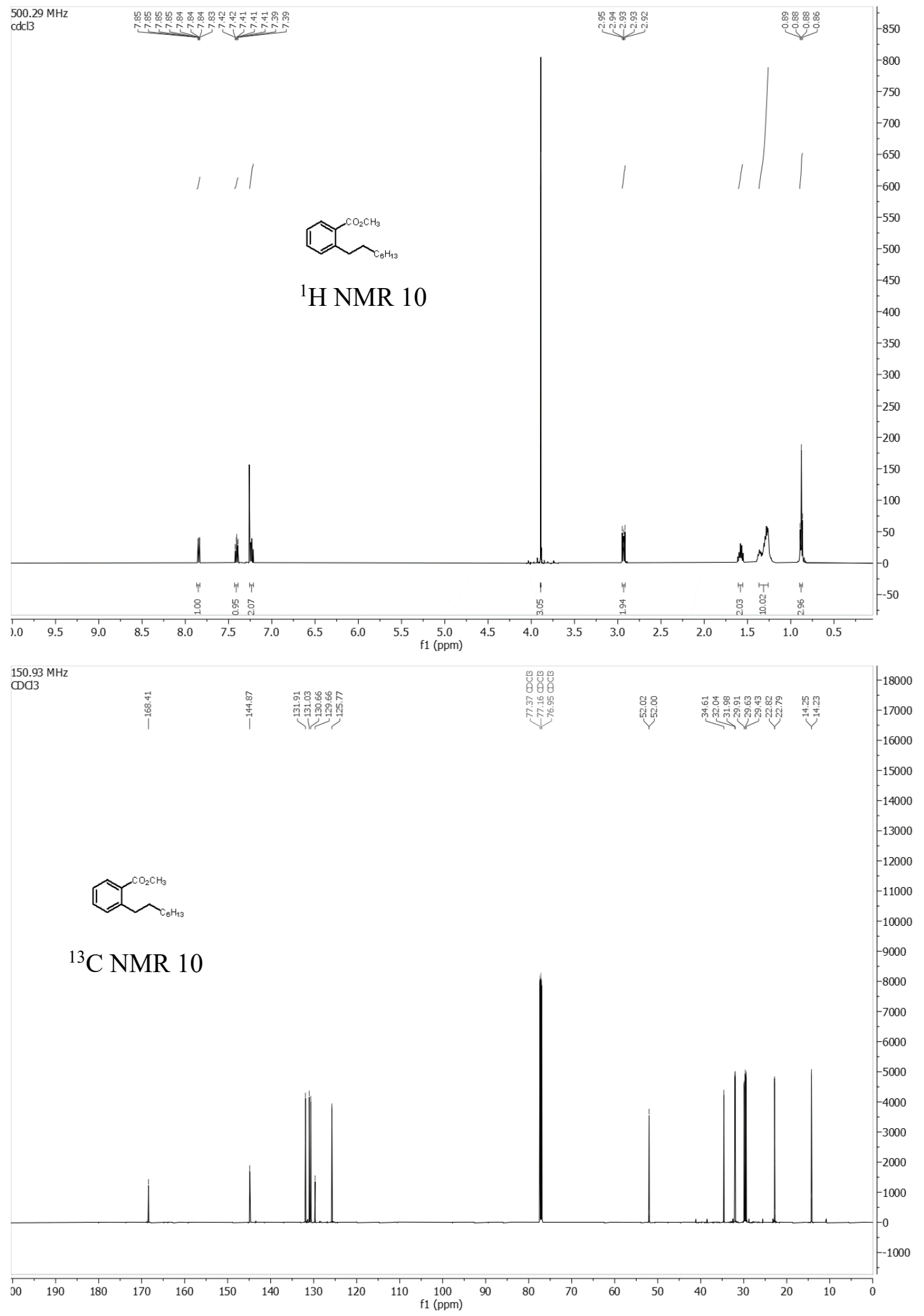


\section{2-methyl-4-oxo-4-(phenethylamino)butanoic acid (30):}
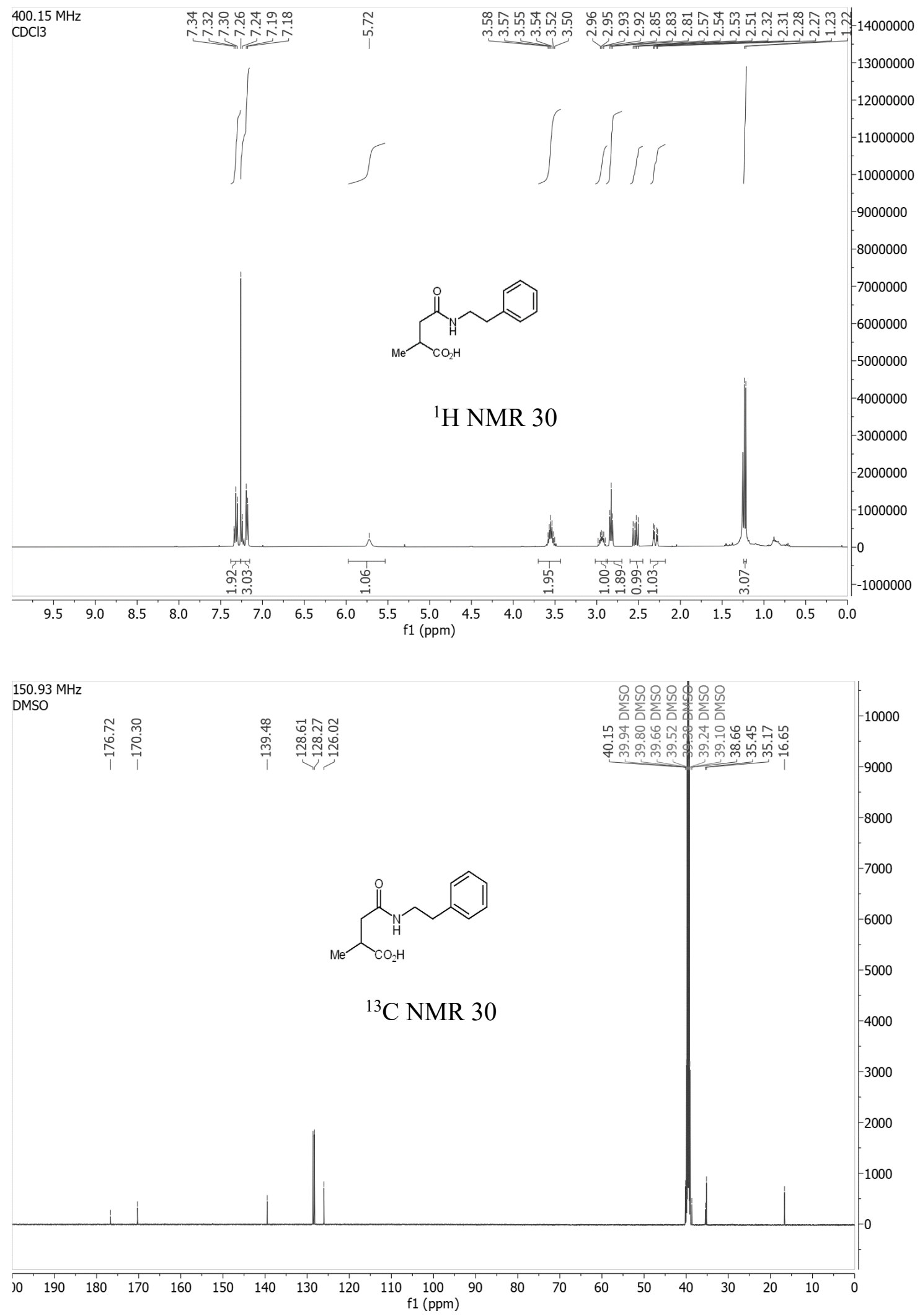


\section{3-cyano-2-methylpropanoic acid (31):}
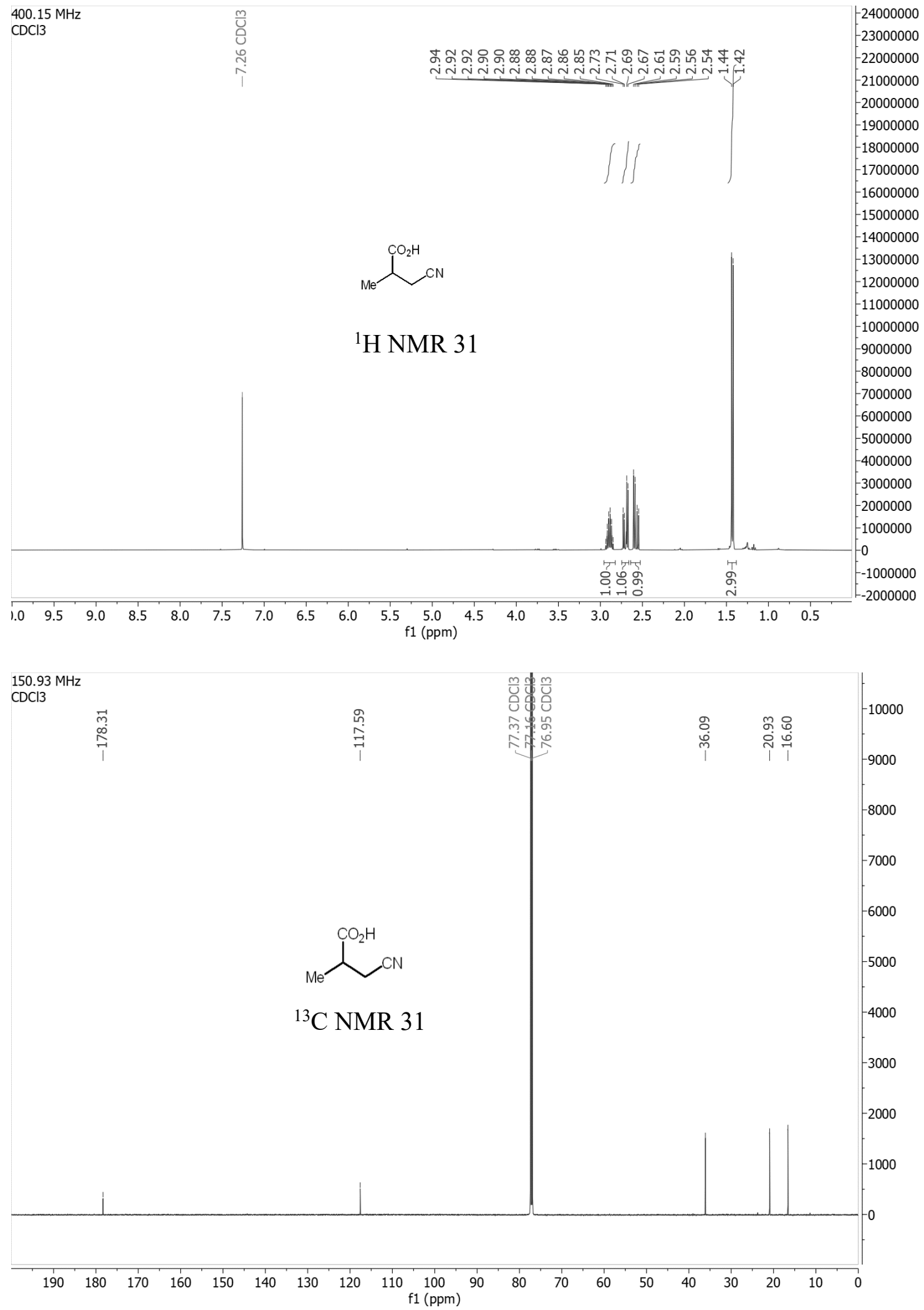


\section{2-cyclopropyl-4-ethoxy-4-oxobutanoic acid (32):}
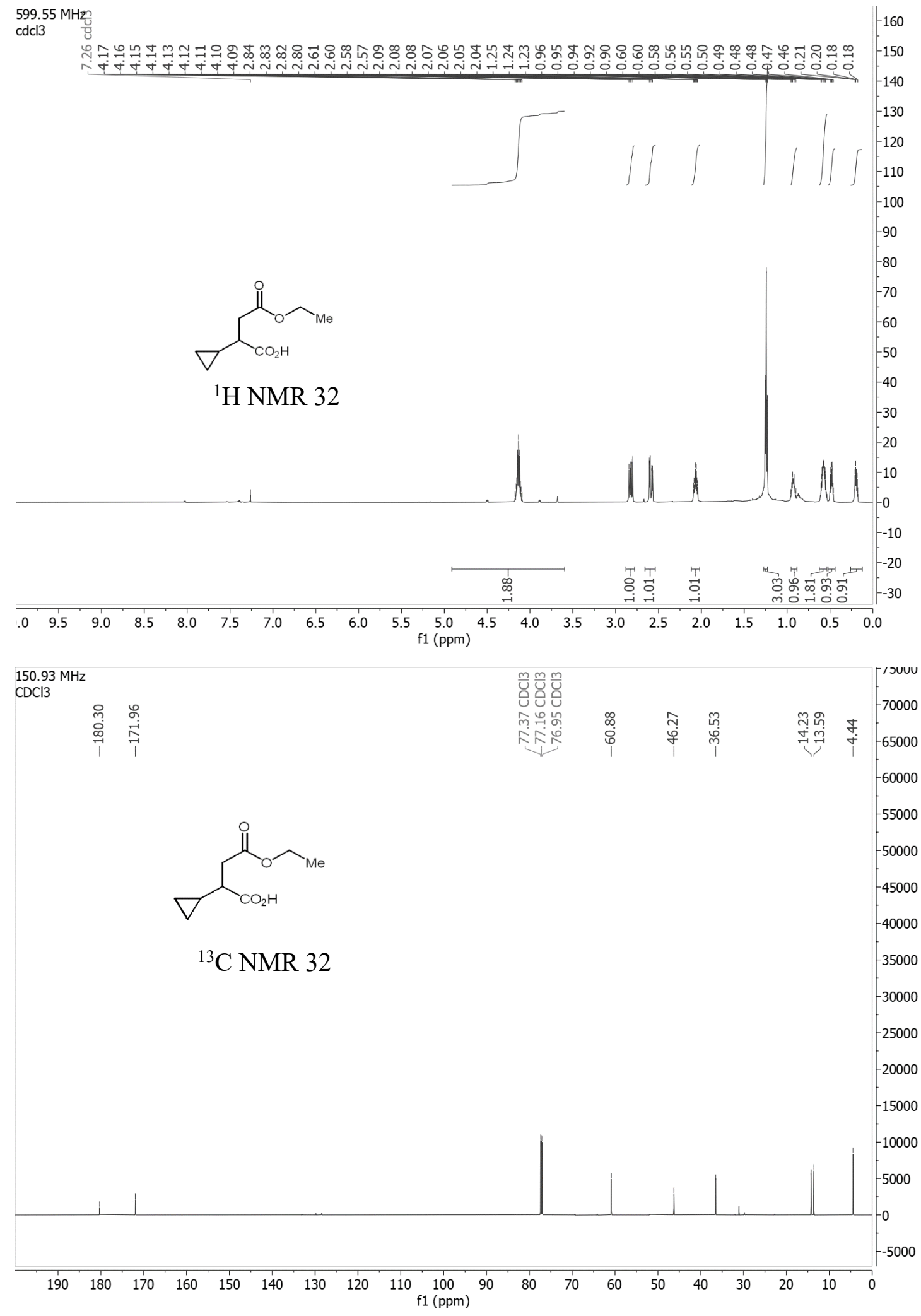


\section{(E)-1-phenylnon-6-en-4-one (33):}
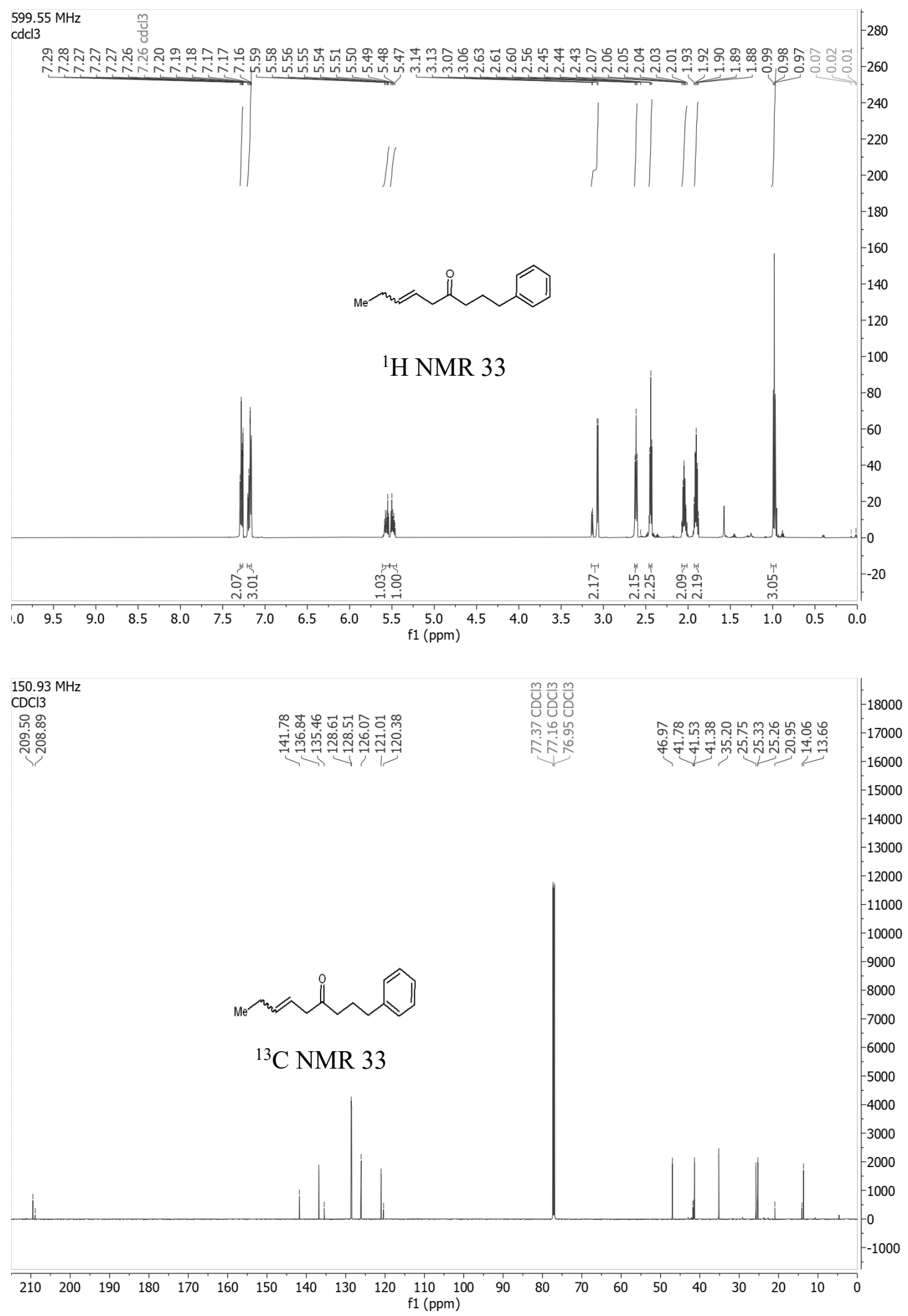